\title{
The Kosterlitz-Thouless Transition in Two-Dimensional Abelian Spin Systems and the Coulomb Gas
}

\author{
Jürg Fröhlich ${ }^{1 \star}$ and Thomas Spencer ${ }^{2, \star \star}$ \\ 1 Institut des Hautes Études Scintifiques, 35, Route de Chartres, F-91440 Bures-sur-Yvette, France \\ 2 Courant Institute of Mathematical Sciences, New York University, 251 Mercer Street, New York, \\ NY 10012, USA
}

\begin{abstract}
We rigorously establish the existence of a Kosterlitz-Thouless transition in the rotator, the Villain, the solid-on-solid, and the $\mathbb{Z}_{n}$ models, for $n$ large enough, and in the Coulomb lattice gas, in two dimensions. Our proof is based on an inductive expansion of the Coulomb gas in the sine-Gordon representation, extending over all possible distance scales, which expresses that gas as a convex superposition of dilute gases of neutral molecules whose activities are small if $\beta$ is sufficiently large. Such gases are known not to exhibit screening. Abelian spin systems are related to a Coulomb gas by means of a duality transformation.
\end{abstract}

\section{Introduction}

\subsection{General Remarks}

In this paper we rigorously establish the Kosterlitz-Thouless transition [1] in a class of two dimensional models including the plane rotator, the $\mathbb{Z}_{n}$-model for $n$ sufficiently large and the lattice Coulomb gas. These results and a brief sketch of the proof have already appeared in [2]. Our methods extend to higher dimensional abelian spin systems, abelian lattice gauge theories and to the onedimensional Ising model with $1 / r^{2}$ interaction. Details of these extensions will appear elsewhere.

All the models we shall analyze are known to have a high temperature phase with exponentially decaying truncated correlations. For example, the Coulomb gas has a high temperature, low density plasma phase characterized by exponential Debye screening [3]. The Kosterlitz-Thouless transition is one from a high temperature phase to a low temperature phase characterized by scaling and a power law fall-off of correlations. In this paper we shall prove the following results:

* Address after Aug. 1982: Physics Dept., ETH, Zürich, Switzerland

$\star \star$ Work supported in part by Grant NSF-DMR 79-04355 and 81-00417 
1. at low temperature, $T$, the spin-spin correlations in the two-dimensional plane rotator and Villain model have a power fall-off;

2. in the two-dimensional Coulomb gas, the sum rule ${ }^{1}$ for the two-point correlation function of the charge density typical for Debye screening is violated, and the correlation between two opposite, fractional charges in this gas have power fall-off, i.e. fractional charges are not screened, provided the temperature and density are sufficiently small;

3. at high temperature, the expectation of $(\phi(0)-\phi(x))^{2}$ in the discrete Gaussian model, (the dual of the Villain model) and in the solid-on-solid model diverges like $\ln |x|$, as $|x| \rightarrow \infty$;

4. for $n$ large enough, there exist temperatures $T_{c}>0$ and $\bar{T}_{c}>T_{c}$ such that, for $I_{c}<T<\bar{T}_{c}$, the $\mathbb{Z}_{n}$-model has a massless, intermediate phase.

Although we shall only consider lattice models, our methods extend e.g. to the regularized continuum Coulomb gas in two dimensions.

In order to heuristically describe the mechanism behind the KosterlitzThouless transition, let us consider the two-dimensional Coulomb gas. In two dimensions the Coulomb potential behaves like $(2 \pi)^{-1} \log (1 / r)$, for large distances, $r$. This potential tends to bind oppositely charged particles into dipoles or more generally into neutral multipoles. More concretely, consider two oppositely charged particles separated by a distance $\ell$. The Boltzmann factor at temperature $T$ for such a configuration, in the absence of other particles, is

$$
B \propto \exp \left[-\frac{\beta}{2 \pi} \ln (\ell+1)\right], \quad \beta=(k T)^{-1} .
$$

However, if $\beta$ is not sufficiently large, the dipole can break up due to entropy effects. The entropy of such a dipole configuration is roughly

$$
S=\ln (\ell A)+\text { const, }
$$

where $\ell$ estimates the number of possible positions of the negative charge, given the position of the positive charge, and $A$ is the area over which the positive charge may range, namely $A \approx \ell^{2}$. Thus we have

$$
e^{S} B \approx \ell^{3} e^{-(\beta / 2 \pi) \ln (\ell+1)} .
$$

When $\beta>8 \pi$, the right side of (3) is summable in $\ell$, so that the probability that a dipole will break up is zero. Thus we conclude that, for large $\beta$, the Coulomb gas looks like a dilute gas of dipoles where long dipoles are unlikely. In Sects. 2-4 of this paper we derive a mathematical identity which expresses the Coulomb gas as a convex combination of neutral multipole gases with small effective activities when $\beta$ is large. This identity is formulated in the sine-Gordon representation. In this representation neutral multipole gases are formally invariant under the continuous symmetry $\phi \rightarrow \phi+$ const, and a Mermin type argument can be applied to prove the absence of exponential screening for such dilute gases. See [5] for a detailed analysis of dipole gases.

The plane rotator and other models analyzed in this paper can be expressed, by a duality transformation, as gases of particles interacting with Coulomb-like

1 We refer here to the Stillinger-Lovett sum rule, see [4] 
forces. Our analysis of these models will follow quite closely that of the Coulomb gas.

Our paper is organized as follows: In Sects. 1.2 and 1.3 the Coulomb gas is described and expressed in the sine-Gordon variables. Our main results on the Coulomb gas and the solid-on-solid model are stated in Sect. 1.3. In Sect. 1.4, we present our results for the plane rotator and the $\mathbb{Z}_{n}$-models. A brief outline of our methods is given in Sect. 1.5. Sections 2-4 form the technical core of our paper. In these sections we establish an identity expressing Coulomb gases as a convex combination of neutral multipole gases. We obtain bounds on the entropy of neutral multipoles (Sect. 2) by a purely combinatorial argument. Our bounds on the effective activity, proved in Sects. 3 and 4, follow from a kind of generalized electrostatic inequality. These identities and estimates are applied in Sects. 5-7 to the various models discussed above. Section 5 is devoted to the Coulomb gas and to the Villain approximation of the plane rotator. Sections 6 and 7 provide a technical refinement of the arguments presented in Sect. 5. We treat the plane rotator in Sect. 6 and the $\mathbb{Z}_{n}$ - and solid-on-solid models in Sect. 7.

\subsection{The Coulomb Gas}

We consider a system of classical particles with electric charge \pm 1 whose possible positions range over a finite array of sites, $\Lambda$, contained in the simple square lattice $\mathbb{Z}^{2}$, (respectively in the lattice $\left.\mathbb{Z}^{v}\right)^{2}$. These particles interact via a two-body Coulomb potential defined as the Green's function, $C(i, j)$, of the finite difference Laplacian, $\Delta$, with some boundary conditions (b.c.) at $\partial \Lambda$, the outer boundary of $\Lambda$. We can analyze the following b.c.:

i) "Free" b.c., i.e. $C(i, j) \equiv C(i-j)$ is the Green's function of the finite difference Laplacian on $\mathbb{Z}^{2}$, but $i$ and $j$ are confined to the region $\Lambda$.

ii) Dirichlet b.c., i.e. $\Delta$ is the finite difference Laplacian with 0 Dirichlet data at $\partial \Lambda$, and $C(i, j)$ its Green's function. Thus

$$
C(i, j)=0 \text { if } i \notin \Lambda \text { or } j \notin \Lambda \text {. }
$$

iii) Periodic b.c.; see Sect. 1.3.

Free b.c. correspond, physically, to putting the particles into a container $A$ with perfectly insulating walls, whereas Dirichlet b.c. correspond to perfectly conducting walls. Usually free or periodic b.c. will be imposed, as they are somewhat simpler technically. (With some technical complications, our methods could be extended to include Dirichlet b.c. as well; see Appendix D.)

A configuration of the gas is given by a function $q_{\Lambda}=\{q(j)\}_{j \in \Lambda}$ with values in $\mathbb{Z}$. Namely, with each site $j \in \Lambda$, one associates the total electric charge, $q(j)$, concentrated at $j$. The energy, $E$, of a configuration $q_{A}$ is the total electrostatic energy of $q_{A}$, self-energies included, i.e.

$$
\begin{aligned}
E\left(q_{\Lambda}\right) & =1 / 2 \sum_{i, j \in A} q(i) q(j) C(i, j) \\
& \equiv 1 / 2\left(q_{\Lambda},(-\Delta)^{-1} q_{\Lambda}\right) .
\end{aligned}
$$

2 The following general remarks apply, of course, to classical Coulomb gases on a lattice of arbitrary dimension $v \geqq 1$, but the main results of this paper concern the two-dimensional gas 
The Boltzmann factor for a configuration $q_{A}$ is

$$
\exp \left[-\beta E\left(q_{\Lambda}\right)\right], \quad \beta=(k T)^{-1} .
$$

The a priori weight of $q_{\Lambda}$ is given by

$$
\prod_{j \in \Lambda} \lambda(q(j))
$$

where $d \lambda(q)$ is a positive (not necessarily finite) measure on $\mathbb{Z}$. The equilibrium state of the system is defined by the usual Gibbs measure, $d \mu$, on the space of all configurations

$$
d \mu\left(q_{\Lambda}\right)=Z_{\Lambda}^{-1} \exp \left[-\beta E\left(q_{\Lambda}\right)\right] \prod_{j \in \Lambda} d \lambda(q(j)),
$$

where

$$
Z_{\Lambda}=\int \exp \left[-\beta E\left(q_{\Lambda}\right)\right] \prod_{j \in \Lambda} d \lambda(q(j))
$$

is the partition function. The finite volume correlations of the charge density are given by

$$
\langle F[\{q(j)\}]\rangle_{\Lambda} \equiv \int F[\{q(j)\}] d \mu\left(q_{\Lambda}\right) .
$$

A thermodynamic limit $\Lambda \uparrow \mathbb{Z}^{2}$ can always be constructed by a compactness argument.

Examples. 1) The hard core gas:

$$
\lambda(q)= \begin{cases}1, & q=0 \\ z / 2, & q= \pm 1 \\ 0, & \text { otherwise. }\end{cases}
$$

In this gas at most one particle can occupy a lattice site, and the bare activity of the particle is $z / 2$.

2) The standard gas:

$$
\lambda(q)=\frac{1}{2 \pi} \int_{0}^{2 \pi} \exp (z \cos \theta) \cos (q \theta) d \theta
$$

for $q \in \mathbb{Z}$, and $z / 2$ is the bare activity. Note that, as $z \rightarrow \infty, \lambda(q) / \lambda(0) \rightarrow 1$, and we obtain

3) The "Villain gas":

$$
\lambda(q)=1 \text { for all } q \in \mathbb{Z} .
$$

In the case of Examples 2 or 3 the thermodynamic limit is known to be independent of the subsequence, as $\Lambda \uparrow \mathbb{Z}^{2}$. See [6] for details.

All the a priori measures we shall study will be assumed to satisfy Condition $\lambda$ : Condition $\lambda$ holds if and only if for all $q \in \mathbb{Z}$,

a) $\lambda(q)=\lambda(-q)$; (charge conjugation invariance).

b) $|\lambda(q)| \leqq$ const $\exp \left[(\alpha+\varepsilon \beta) q^{2}\right], \varepsilon<1 / 16$; (low density condition).

Remark. If our low density condition b) fails, then the gas may be in a crystalline phase. The existence of such a phase for high density and low temperature was proved in [7]. 


\subsection{The Sine-Gordon Representation of the Coulomb Gas}

In this section we discuss the Fourier transform of the Gibbs measure we defined in (1.7) with respect to the charge variables $\{q(j)\}$. This provides a technically extremely useful representation of the Coulomb gas, known as sine-Gordon, or Siegert representation $[5,8,9]$. We denote by $d \mu_{\beta, \Omega}$ the Gaussian measure with mean 0 and covariance $\beta\left(-\Delta_{\Omega}\right)^{-1}$, where $\Delta_{\Omega}$ is the finite difference Laplacian with 0 -Dirichlet data at the boundary $\partial \Omega$ of some finite region $\Omega$.

Clearly

$$
\int e^{i \phi(\varrho)} d \mu_{\beta, \Omega}(\phi)=\exp \left[-(\beta / 2)\left(\varrho,\left(-\Delta_{\Omega}\right)^{-1} \varrho\right)\right],
$$

where $\phi(\varrho)=\sum_{j} \phi(j) \varrho(j)$, and $\varrho$ is a function of finite support in $\Omega$. It is easy to see that, for a fixed function $\varrho$ of finite support,

$$
\lim _{\Omega \uparrow \mathbb{Z}^{2}} \int e^{i \phi(\varrho)} d \mu_{\beta, \Omega}(\phi)=\left\{\begin{array}{lll}
\exp [-\beta E(\varrho)], & \text { if } & Q(\varrho) \equiv \sum \varrho(j)=0 \\
0, & \text { if } & Q(\varrho) \neq 0
\end{array}\right.
$$

Here $E(\varrho)$ is the electrostatic energy of $\varrho$ corresponding to free boundary conditions. Note that if $\sum \varrho(j) \neq 0, E_{\Omega}(\varrho) \rightarrow \infty$ as $\Omega \uparrow \mathbb{Z}^{2}$, because $-\Delta(p)^{-1} \approx p^{-2}$ is not integrable about $p=0$. Formula (1.14) also holds for the case where periodic boundary conditions are imposed on the boundary of the rectangles $\Omega$. Let $d \mu_{\beta}$ $=\lim d \mu_{\beta, \Omega}$, as $\Omega \uparrow \mathbb{Z}^{2}$.

By (1.8) and (1.14) the partition function $Z_{\Lambda}$ of the Coulomb gas is given by

$$
\begin{aligned}
Z_{\Lambda} & =\int \exp \left[-\beta E\left(q_{\Lambda}\right)\right] \prod_{j \in \Lambda} d \lambda(q(j)) \\
& =\int \prod_{j \in \Lambda}\left\{\int e^{i \phi(j) q(j)} d \lambda(q(j))\right\} d \mu_{\beta}(\phi) \\
& =\int \prod_{j \in \Lambda} \hat{\lambda}(\phi(j)) d \mu_{\beta}(\phi),
\end{aligned}
$$

where $\hat{\lambda}$ is the Fourier transform of $d \lambda$.

Let $\langle\cdot\rangle_{\Lambda}^{\phi}(\beta, \lambda)$ be the expectation with respect to the measure (not necessarily positive)

$$
Z_{\Lambda}^{-1} \prod_{j \in \Lambda} \hat{\lambda}(\phi(j)) d \mu_{\beta}(\phi)
$$

By definition of $\hat{\lambda}$ and $\langle\cdot\rangle^{\phi}$ the charge density correlations are given by

$$
\left\langle\prod_{j} q(j)^{n_{j}}\right\rangle_{A}(\beta, \lambda)=\left\langle\prod_{j} \hat{\lambda}(\phi(j))^{-1}\left\{\left(-i \frac{\partial}{\partial \phi(j)}\right)^{n_{j}} \hat{\lambda}\right\}(\phi(j))\right\rangle_{\Lambda}^{\phi}(\beta, \lambda) .
$$

If no confusion results we set $\langle\cdot\rangle^{\phi}=\langle\cdot\rangle$. By integration by parts in a periodic box one can easily establish (see [5])

$$
\left\langle|\hat{\phi}(k)|^{2}\right\rangle(\beta, \lambda)=\beta \hat{C}(k)-\beta^{2} \hat{C}(k)^{2}\left\langle|\hat{q}(k)|^{2}\right\rangle(\beta, \lambda)
$$

for $k \neq 0$. If $\langle\cdot\rangle^{\phi}$ is a positive measure, the left hand side of (1.17) is positive, and we conclude that

$$
\left\langle\left|\hat{q}(k)^{2}\right|\right\rangle(\beta, \lambda) \leqq \beta^{-1} \hat{C}(k)^{-1} .
$$


Now let

$$
\begin{aligned}
M(\beta) & \equiv-\sum_{j=\left(j_{1}, j_{2}\right)}\langle q(0) q(j)\rangle(\beta, \lambda) \cdot\left(j_{1}^{2}+j_{2}^{2}\right) \\
& =\left.\left(\frac{\partial^{2}}{\partial k_{1}^{2}}+\frac{\partial^{2}}{\partial k_{2}^{2}}\right)\left\langle|\hat{q}(k)|^{2}\right\rangle(\beta, \lambda)\right|_{k=0} .
\end{aligned}
$$

If exponential Debye screening holds, then $\left\langle|\hat{q}(k)|^{2}\right\rangle$ is analytic in $k$ near 0 , and a well-known $\lambda$-independent sum rule $[4,5]$ asserts that $M(\beta)=2 \beta^{-1}$. By expanding the right side of (1.17) in a power series in $k$ we see that $\left\langle|\hat{\phi}(k)|^{2}\right\rangle$ is bounded at $k=0$ if $M(\beta)=2 \beta^{-1}$.

In Sect. 5 we prove the following theorem.

Theorem A. If $d \lambda$ satisfies condition $\lambda$ then for $\beta$ sufficiently large there exists a constant $c_{1}>0$ such that for $\phi(f) \equiv \sum \phi(j) f(j)$

whenever $\sum f(j)=0$.

$$
\left\langle|\phi(f)|^{2}\right\rangle \geqq c_{1}\left\langle f,-\Delta^{-1} f\right\rangle
$$

Remarks. Theorem A implies that for $k \neq 0\left\langle|\hat{\phi}(k)|^{2}\right\rangle \geqq c_{1} k^{-2}$, which is singular near $k=0$. Thus the sum rule for Debye screening must be violated.

If $\lambda(q)=1$, for all $q \in \mathbb{Z}$, we see that $\hat{\lambda}(\phi(j))=\sum_{n} \delta(\phi(j)-2 \pi n)$, and $\langle\cdot\rangle^{\phi}$ is, by definition, the discrete Gaussian model. If we now let $f(j)=\delta_{x, j}-\delta_{y, j}$, Theorem A asserts that

$$
\left\langle[\phi(x)-\phi(y)]^{2}\right\rangle \geqq \pi^{-1} c_{1} \log (|x-y|+1)
$$

for large $\beta$, which proves the existence of a roughening transition for the discrete Gaussian model.

In Sect. 7 we extend these results to the solid-on-solid model [10]. In this model the Gibbs measure is given by

$$
\exp \left\{-\sum_{j, j^{\prime}} \beta\left|\phi(j)-\phi\left(j^{\prime}\right)\right|\right\} \prod_{j} \hat{\lambda}\left(\phi_{j}\right) d \phi_{j}
$$

where the sum is over nearest neighbors. The single spin distribution is again $\hat{\lambda}(\phi)$ $=\sum_{n} \delta(2 \pi n-\phi)$.

We also determine the behavior of the fractional charge correlation defined by

$$
G_{\xi}(x)=\langle\exp (i \xi(\phi(0)-\phi(x)))\rangle,
$$

where $\xi$ is the strength of the fractional charge. Using the sine-Gordon representation (Sect. 1.3) it is easy to see that the log of

$$
G_{\xi, \Lambda}(x)=Z_{\Lambda}^{-1} \int \exp \left[-\beta E\left(q_{\Lambda}+\xi\left(\delta_{j 0}-\delta_{j x}\right)\right)\right] \cdot \prod_{j \in \Lambda} d \lambda(q(j))
$$

measures the average energy required to pump two opposite fractional charges located at 0 and $x$ into the system. Using Jensen's inequality in the $q$ variables we can show that

$$
G_{\xi}(x) \geqq \exp (-\beta[C(0,0)-C(0, x)]) \approx|x|^{-[\beta / 2 \pi]}
$$


(see [5] for details). If screening occurs (e.g. at high temperatures) then it has been shown [3] that

$$
G_{\xi}(x) \rightarrow L>0
$$

exponentially fast. We prove

Theorem B. For $\beta$ sufficiently large and $\lambda$ satisfying condition $\lambda$ we have for $0<\xi<1$

$$
0 \leqq G_{\xi}(x) \leqq \text { const }|x|^{-\beta^{\prime}}
$$

where $\beta^{\prime}=\beta^{\prime}(\beta, \xi) \rightarrow \infty$, as $\beta \rightarrow \infty$, and $G_{\xi}$ is given by (1.23).

\subsection{The Two-Dimensional Villain - and Rotator Model}

The equilibrium states of the Villain and the plane rotator models in zero magnetic field, in a finite region $\Lambda \subset \mathbb{Z}^{2}$, are given, respectively, by

$$
\left.\begin{array}{l}
Z_{\Lambda}^{-1} \prod_{(i j) \subset \Lambda} v_{\beta}\left(\theta_{i}-\theta_{j}\right) \prod_{j \in \Lambda} d \theta_{j}, \\
Z_{\Lambda}^{-1} \prod_{(i j) \subset \Lambda} r_{\beta}\left(\theta_{i}-\theta_{j}\right) \prod_{j \in \Lambda} d \theta_{j},
\end{array}\right\}
$$

where $\prod_{(i j) \subset A}$ extends over all nearest neighbor pairs in $\Lambda$, and

$$
v_{\beta}(\theta)=\sum_{m \in \mathbb{Z}} \exp \left[-(\beta / 2)(\theta+2 \pi \mathrm{m})^{2}\right]
$$

is the periodized Gaussian, and

$$
r_{\beta}(\theta)=\exp (\beta \cos \theta) .
$$

The variables $\theta_{j}, j \in \Lambda$, are angular variables in terms of which the original spin variables are expressed by

$$
\mathbf{S}_{j}=\left(\cos \theta_{j}, \sin \theta_{j}\right) \text {. }
$$

The expectation in the measures defined in (1.26) is denoted by $\langle\cdot\rangle_{A}(\beta)$. The thermodynamic limit, $\langle-\rangle(\beta)$, of the states $\left\{\langle\cdot\rangle_{\Lambda}(\beta)\right\}$ is known to exist, a consequence of correlation inequalities [11], and to be the unique translationinvariant Gibbs state of the system [12], for all values of $\beta$. It is invariant under the continuous symmetry $\theta_{j} \rightarrow \theta_{j}+\alpha, \bmod 2 \pi$, where $\alpha$ is an arbitrary constant. By the $\theta \rightarrow-\theta$ symmetry.

$$
\left\langle\mathbf{S}_{j} \cdot \mathbf{S}_{\ell}\right\rangle(\beta)=\left\langle\cos \left(\theta_{j}-\theta_{\ell}\right)\right\rangle(\beta)=\left\langle e^{i\left(\theta_{j}-\theta_{\ell}\right)}\right\rangle(\beta) .
$$

By using e.g. a standard high temperature expansion, one shows that

$$
\left\langle\mathbf{S}_{j} \cdot \mathbf{S}_{\ell}\right\rangle(\beta) \rightarrow 0, \quad \text { as } \quad|j-\ell| \rightarrow \infty
$$

exponentially fast, provided $\beta$ is small enough. (It is shown in [13] that for the rotator model (1.30) holds if $\beta<2 \beta_{\text {crit }}$ (Ising). Further upper bounds on critical temperatures are mentioned in [5].) McBryan and Spencer [14] have shown that, for all $\beta$ and for every $\varepsilon>0$, there exists a finite constant $K=K(\varepsilon, \beta)$ such that

$$
0 \leqq\left\langle\mathbf{S}_{j} \cdot \mathbf{S}_{\ell}\right\rangle(\beta) \leqq K(1+|j-\ell|)^{-[1 /(2 \pi+\varepsilon) \beta]} .
$$

One of the main results of our paper is 
Theorem C. In the two-dimensional Villain and plane rotator models, for sufficiently large $\beta$,

$$
\left\langle\mathbf{S}_{j} \cdot \mathbf{S}_{\ell}\right\rangle(\beta) \geqq \operatorname{const}(1+|j-\ell|)^{-\left[1 / 2 \pi \beta^{\prime}\right]},
$$

for some $\beta^{\prime}=\beta^{\prime}(\beta)>1 / 4 \pi$. Moreover $\beta^{\prime}(\beta) \rightarrow \infty$, as $\beta \rightarrow \infty$.

The proof appears in Sects. 5 and 6.

Next, we define the usual disorder operator. Let $x$ be the site $(n, 0)$, and define

$$
D_{0 x}^{\xi}=\prod_{m=1}^{n} \frac{f_{\beta}\left(\theta_{(m, 1)}-\theta_{(m, 0)}+2 \pi \xi\right)}{f_{\beta}\left(\theta_{(m, 1)}-\theta_{(m, 0)}\right)},
$$

where $f_{\beta}=r_{\beta}$, respectively $v_{\beta}$, and $\xi \in(0,1)$. It follows from a high temperature expansion that

$$
\left\langle D_{0 x}^{\xi}\right\rangle(\beta) \geqq \text { const }>0,
$$

uniformly in $|x|$, when $\beta$ is sufficiently small.

In Sect. 5 we prove the following result for the Villain model.

Theorem D. For each $\xi \in(0,1)$ there exists some finite $\beta_{0}(\xi)$ such that for $\beta>\beta_{0}(\xi)$

$$
\left\langle D_{0 x}^{\xi}\right\rangle(\beta) \leqq \operatorname{const}(1+|x|)^{-\xi^{2} \beta^{\prime \prime}},
$$

for some positive $\beta^{\prime \prime}=\beta^{\prime \prime}(\xi, \beta)$.

Our proofs of Theorems C and D proceed by studying the models dual to the Villain, respectively the plane rotator models which are obtained by Fourier transformation in the angular variables. The Fourier coefficients of $v_{\beta}$ and $r_{\beta}$ are given by

$$
\left.\begin{array}{l}
\hat{v}_{\beta}(\phi)=\text { const } \exp \left[-(1 / 2 \beta) \phi^{2}\right], \\
\hat{r}_{\beta}(\phi)=I_{\phi}(\beta),
\end{array}\right\} \phi \in \mathbb{Z},
$$

where $I_{\phi}(\beta)$ is the $\phi^{\text {th }}$ modified Bessel function. Let $I_{\beta}(\phi)=\hat{v}_{\beta}(\phi)$ or $\hat{r}_{\beta}(\phi)$. Let $\Lambda$ be some finite region in the dual lattice. We define a statistical weight of a configuration $\{\phi(j)\}_{j \in \Lambda}$ of integer-valued spins, $\phi(j)$, by

$$
Z_{\Lambda}^{-1} \prod_{\Lambda} I_{\beta}(\phi(i)-\phi(j))
$$

where $\prod_{\Lambda}$ extends over all nearest neighbor pairs $(i j)$ with $(i j) \cap \Lambda \neq \emptyset$ and $\phi(\ell)=0$, for $\ell \notin \Lambda$.

Let $\langle\cdot\rangle_{A}^{*}(\beta)$ be the expectation determined by the weight $(1.35)$, and let $\langle\cdot\rangle^{*}(\beta)$ denote its thermodynamic limit. This is the Gibbs state of the model dual to the Villain, the rotator model, respectively. We define

$$
\hat{D}_{0 x}^{\xi}=\prod_{m=0}^{n-1} \frac{I_{\beta}(\phi(m, 0)-\phi(m, 1)+\xi)}{I_{\beta}(\phi(m, 0)-\phi(m, 1))},
$$

where $x=(n, 0)$, and $\xi$ is some integer. It is well known that

$$
\left\langle e^{i \xi\left(\theta_{0}-\theta_{x}\right)}\right\rangle(\beta)=\left\langle\hat{D}_{0 x}^{\xi}\right\rangle^{*}(\beta),
$$

in particular

$$
\left\langle\mathbf{S}_{0} \cdot \mathbf{S}_{x}\right\rangle(\beta)=\left\langle e^{i\left(\theta_{0}-\theta_{x}\right)}\right\rangle(\beta)=\left\langle\hat{D}_{0 x}^{1}\right\rangle^{*}(\beta)
$$


The relation dual to (1.37) is

$$
\left\langle D_{0 x}^{\xi}\right\rangle(\beta)=\left\langle e^{2 \pi i \xi\left(\phi_{0}-\phi_{x}\right)}\right\rangle *(\beta) .
$$

These relations can be extended to general correlation functions of products of spin variables and disorder operators. See Appendix A for a proof of these identities. We shall see that (1.37) is important for our proof of Theorem C.

We also obtain results for the $\mathbb{Z}_{n}$-models which are defined by the Hamiltonian

$$
H_{n}(\theta)=-\sum_{(i j) \subset \Lambda} \cos \left(\theta_{i}-\theta_{j}\right)-h \sum_{j \in \Lambda} \cos \left(n \theta_{j}\right),
$$

as $h \rightarrow \infty$. The corresponding states defined by

$$
\langle\cdot\rangle^{(n)}(\beta) \equiv \lim _{h \rightarrow \infty}\langle\cdot\rangle^{(n)}(\beta, h)=\lim _{n \rightarrow \infty} Z_{A}^{-1} \int e^{-\beta H_{n}(\theta)}-\prod_{j \ni A} d \theta_{j}
$$

are known to have a thermodynamic limit independent of $\{\Lambda\}$, as $\Lambda \uparrow \mathbb{Z}^{2}$, by Ginibre's correlation inequalities. The spin-spin correlation is also monotone increasing in $h$ and $\Lambda$. Hence

$$
\left\langle e^{i \xi\left(\theta_{0}-\theta_{x}\right)}\right\rangle^{(n)}(\beta) \geqq\left\langle e^{i \xi\left(\theta_{0}-\theta_{x}\right)}\right\rangle^{(n)}(\beta, 0),
$$

which is bounded below by a power law by Theorem $\mathrm{C}$ when $\beta \gg 1$. Note that, as $h \rightarrow \infty, \theta$ is constrained to take discrete value $\frac{k}{n} 2 \pi, k=1,2, \ldots, n$.

The next theorem establishes the existence of a massless intermediate phase for large $n$.

Theorem E. Given $\beta$ and $q \in(0,1)$, there exists $n_{0}=n_{0}(\beta, q)<\infty$ such that for all $n \geqq n_{0}$ and all integers $\xi$ with $\min (\xi / n, 1-\xi / n) \geqq q$

$$
\left\langle e^{i \xi\left(\theta_{0}-\theta_{x}\right)}\right\rangle^{(n)}(\beta)<\operatorname{const}(1+|x|)^{-\left[\xi^{2} / 2 \pi \beta^{\prime \prime}\right]},
$$

for some $\beta^{\prime \prime}=\beta^{\prime \prime}(\beta, q)<\infty$.

A related result was proved by Elitzur et al. [15] for the Villain approximation using duality and correlation inequalities. Their proof assumed Theorem $\mathrm{C}$.

\subsection{Outline of the Proof}

We conclude this section with an outline of our proof of Theorem A. For simplicity we shall consider the special case of the hard core Coulomb gas whose finite volume expectation is given by

$$
\langle\cdot\rangle_{\Lambda}=Z_{\Lambda}^{-1} \int \cdot \prod_{j \in \Lambda}(1+z \cos \phi(j)) d \mu_{\beta}(\phi) .
$$

Let us estimate $\left\langle e^{\varepsilon \phi(f)}\right\rangle$, from below where, for example, $f(j)=\delta_{0 j}-\delta_{x j}$. To do this we make a change of variables

where

$$
\phi(j) \rightarrow \phi(j)+\sigma(j)
$$

$$
\sigma(j)=-\beta \varepsilon\left\langle\delta_{j}, \Delta^{-1} f\right\rangle
$$


We then obtain the identity:

where

$$
\left\langle e^{\varepsilon \phi(f)}\right\rangle e^{(\beta / 2) \varepsilon^{2}\left\langle f, \Delta^{-1} f\right\rangle}=Z_{\Lambda}(0)^{-1} Z_{\Lambda}(\sigma),
$$

$$
Z_{\Lambda}(\sigma) \equiv \int \prod_{j \in \Lambda}(1+z \cos (\phi(j)+\sigma(j))) d \mu_{\beta}(\phi)
$$

We would like to apply Jensen's inequality to obtain a bound from below on $Z^{-1}(0) Z(\sigma)$. The problem is that $\sigma(j)$ is not small. However if $|j-k|=L$, then

$$
|\sigma(j)-\sigma(k)| \leqq \operatorname{const} L\left(\frac{1}{|j|+1}+\frac{1}{|j-x|+1}\right) .
$$

Our first major step is to arrange for differences of this type to occur. In Sect. 2 we prove the identity

$$
\begin{aligned}
\prod & {[1+z \cos (\phi(j)+\sigma(j))] } \\
= & \sum_{\mathscr{N}} c_{\mathscr{N}} \prod_{\varrho \in \mathscr{N}}[1+K(\varrho) \cos (\phi(\varrho)+\sigma(\varrho))], \quad c_{\mathscr{N}}>0,
\end{aligned}
$$

where $\mathscr{N}$ is a collection of neutral charge densities, $\varrho$ [i.e. $\left.\sum \varrho(j)=0\right]$, and $\phi(\varrho) \equiv \sum \phi(j) \varrho(j)$. More precisely, $\mathscr{N}$ contains at most one charged density $\varrho_{c}$ which we shall show has 0 effective activity, since its self-energy is infinite. By (1.48) we see that $\sigma(\varrho)$ is fairly small if $\varrho$ is neutral and supported away from 0 and $x$. The constants $K(\varrho)$ are closely related to the entropy of the charge density $\varrho$ and are large for a big multipole $\varrho$. Our next major step is to offset the entropy by extracting part of the Boltzmann factor. In Sect. 4 we prove that

$$
\begin{aligned}
Z_{\mathcal{N}}(\sigma) & \equiv \int \prod_{\varrho \in \mathcal{N}}(1+K(\varrho) \cos (\phi(\varrho)+\sigma(\varrho))) d \mu_{\beta}(\phi) \\
& =\int \prod_{\varrho \in \mathcal{N}}\left(1+K(\varrho) e^{-\beta E_{1 \mathrm{oc}}(\varrho)} \cos (\phi(\bar{\varrho})+\sigma(\varrho))\right) d \mu_{\beta}(\phi),
\end{aligned}
$$

where $\varrho$ is a new effective neutral charge density corresponding to $\varrho$ and $E_{1 \mathrm{loc}}(\varrho)$ is roughly the electrostatic energy of $\varrho$. This result follows from elementary, but lengthy electrostatic identities.

In Sect. 3 we prove the key bound on the effective activity $z$ defined by

$$
z(\beta, \varrho) \equiv K(\varrho) e^{-\beta E_{1 o c}(\varrho)} \leqq \exp [(-\beta c+b) \log (d(\varrho)+1)],
$$

where $c$ and $b$ are positive constants independent of $\beta$, and $d(\varrho)$ is the diameter of $\varrho$. For large $\beta,(1.51)$ implies large multipoles are unlikely. It is now straightforward (see Sect. 5) to apply Jensen's inequality and the double angle formula to show that

$$
\begin{aligned}
Z_{\mathscr{N}}(0)^{-1} Z_{\mathscr{N}}(\sigma) & \geqq \exp \left[- \text { const } \sum_{\varrho \in \mathcal{N}} z(\beta, \varrho) \sigma(\varrho)^{2}\right] \\
& \geqq \exp \left[\gamma(\beta) \varepsilon^{2}\left(f, \Delta^{-1} f\right)\right]
\end{aligned}
$$

for each $\mathscr{N}$ appearing in (1.49). The constant $\gamma(\beta) \rightarrow 0$, as $\beta$ becomes large. If we combine (1.46), (1.50), and (1.52) we see that

$$
\left\langle e^{\varepsilon \phi(f)}\right\rangle>\exp \left[-\varepsilon^{2}(\beta / 2-\gamma(\beta))\left\langle f, \Delta^{-1} f\right\rangle\right] .
$$

Theorem A now follows by expanding up to second order in $\varepsilon$, dividing by $\varepsilon^{2}$ and taking $\varepsilon \rightarrow 0$. 


\section{An Expansion in Terms of Neutral Multipole Ensembles and Entropy Estimates}

In this section we prove our main combinatorial theorem which expresses expectations in the two-dimensional Coulomb gas as convex combinations of expectations in dilute gases of neutral multipoles of variable size. Our principal estimate is on the entropy, respectively bare activity, of the neutral multipoles which constitute the resulting gases.

First, we derive an expansion for the partition function, see Subsects. 2.1-2.4. That expansion also serves as a starting point for estimating the expectation of the disorder parameter (1.36) and of $(\phi(0)-\phi(x))^{2}$; see (1.19). In Subsect. 2.5, we explain how to modify the expansion in order to obtain a suitable representation of the fractional charge correlation, $G_{\xi}(x)$, defined in (1.22).

In Sects. 2-5, we impose free (i.e. insulating) boundary conditions. Our method extends to periodic b.c., as well, but Dirichlet b.c. require some refinements briefly sketched in Appendix D. We recommend that the reader try to absorb the definitions and arguments in this section by representing them graphically.

\subsection{Notation}

Let $\Lambda \subset \mathbb{Z}^{2}$ be a large rectangular array of lattice sites $j \in \mathbb{Z}^{2}$. We confine the positions of the charged particles in the gas to be inside $\Lambda$. A charge density $\varrho$ is an integer-valued function on $\Lambda$ whose value, $\varrho(j)$, at some site $\mathrm{j} \in \Lambda$ indicates the charge concentrated at $j$. The diameter of the support of $\varrho$ is denoted by $d(\varrho)$, and $\operatorname{dist}\left(\varrho_{1}, \varrho_{2}\right)$ is the minimum distance between the supports of two charge densities $\varrho_{1}$ and $\varrho_{2}$.

An ensemble, $\mathscr{E}$, is a collection of charge densities, $\varrho$, whose supports are mutually disjoint. A charge density $\varrho_{1}$ is said to be compatible with an ensemble $\mathscr{E}$ if

$$
\varrho_{1}=\sum_{\varrho \in \mathscr{E}} \varepsilon\left(\varrho_{1}, \varrho\right) \varrho, \quad \text { with } \quad \varepsilon\left(\varrho_{1}, \varrho\right)=0, \pm 1
$$

We say that an ensemble $\mathscr{E}_{1}$ is a parent of an ensemble $\mathscr{E}_{2}, \mathscr{E}_{1} \rightarrow \mathscr{E}_{2}$, iff every charge density $\varrho \in \mathscr{E}_{2}$ is compatible with $\mathscr{E}_{1}$. We say that a density $\varrho$ is a constituent of a density $\varrho_{1}, \varrho \subset \varrho_{1}$, iff supp $\varrho \subset \operatorname{supp} \varrho_{1}, \varrho(j)=\varrho_{1}(j)$ for all $j \in \operatorname{supp} \varrho$.

The total charge, $Q(\varrho)$, of a density $\varrho$ is defined by

$$
Q(\varrho)=\sum_{j} \varrho(j)
$$

A density $\varrho$ is neutral if $Q(\varrho)=0$; otherwise it is said to be charged. We define an area of a density $\varrho$ on various distance scales : Let $A_{n}(\varrho)$ be the minimal number of $2^{n} \times 2^{n}$ squares - i.e. squares with sides containing $2^{n}$ sites - needed to cover supp $\varrho$. Clearly $A_{0}(\varrho)=\operatorname{card}\{\operatorname{supp} \varrho\}$.

An ensemble $\mathscr{E}=\mathscr{E}_{n}$ is called an $n$-ensemble iff for all $\varrho_{1}, \varrho_{2}$ in $\mathscr{E}_{n}, \varrho_{1} \neq \varrho_{2}$,

$$
\operatorname{dist}\left(\varrho_{1}, \varrho_{2}\right)>2^{n} .
$$




\subsection{Main Result}

Theorem 2.1. There exists a family, $\mathscr{F}=\mathscr{F}_{\Lambda}$, of ensembles, $\mathscr{N}$, such that

$$
\prod_{j \in \Lambda}(1+z \cos \phi(j))=\sum_{\mathscr{N} \in \mathscr{F}} c_{\mathscr{N}} \prod_{\varrho \in \mathscr{N}}(1+K(\varrho) \cos \phi(\varrho)),
$$

$\left(\phi(\varrho) \equiv \sum_{j} \phi(j) \varrho(j)\right)$, where $c_{\mathscr{N}}>0$, for all $\mathscr{N} \in \mathscr{F}$, and each $\mathscr{N} \in \mathscr{F}$ has the following properties :

a) all densities $\varrho$ in $\mathcal{N}$ are neutral, i.e. $Q(\varrho)=0$, except possibly one, $\varrho_{c}$, which is charged.

There exist constants $M>1$ and $\alpha, 3 / 2<\alpha<2$, independent of $\mathcal{N}$ such that

$$
\operatorname{dist}\left(\varrho_{1}, \varrho_{2}\right) \geqq M\left[\min \left(d\left(\varrho_{1}\right), d\left(\varrho_{2}\right)\right)\right]^{\alpha},
$$

for all $\varrho_{1}, \varrho_{2}$ in $\mathscr{N}, \varrho_{1} \neq \varrho_{2}$. Moreover, if $\mathscr{N}$ contains a charge density $\varrho_{c}$ with $Q\left(\varrho_{c}\right) \neq 0$

for all $\varrho$ in $\mathscr{N}, \varrho \neq \varrho_{c}$.

$$
\operatorname{dist}\left(\varrho, \varrho_{c}\right) \geqq M d(\varrho)^{\alpha},
$$

c) If $\varrho_{1} \subset \varrho \in \mathscr{N}$ is such that

then $\varrho_{1}$ is charged.

$$
\operatorname{dist}\left(\varrho_{1}, \varrho-\varrho_{1}\right) \geqq 2 M d\left(\varrho_{1}\right)^{\alpha}
$$

Finally, the constants $K(\varrho)$ on the right side of (2.3) satisfy

$$
0<K(\varrho)=z^{A_{0}(\varrho)} e^{S(\varrho)}, \text { with } \quad S(\varrho) \leqq C \cdot A(\varrho),
$$

for some $\varrho$ - and $\mathscr{N}$ - independent constant $C$, where

with

$$
A(\varrho)=\sum_{k=0}^{n(\varrho)} A_{k}(\varrho),
$$

for all $\varrho \neq \varrho_{c}$.

$$
n(\varrho) \text { the smallest integer } \geqq \ln _{2}\left(M d(\varrho)^{\alpha}\right) \text {, }
$$

Remarks. 1. $S(\varrho)$ can be thought of as the entropy of a multipole with charge density $\varrho$.

2. The constants $M$ and $\alpha$ above will be chosen later. We shall see that $\frac{3}{2}<\alpha<2$ is the admissible range of $\alpha$. Property b) of the ensembles $\mathscr{N} \in \mathscr{F}$, asserted in Theorem 2.1, ensures that the gases with ensembles $\mathscr{N}$ are sufficiently dilute to enable us to extract the selfenergy factor $\exp [-\operatorname{const} \beta E(\varrho)]$ of the neutral charge density $\varrho ;\left(\alpha>\frac{3}{2}\right.$ is a sufficient condition for this to be possible). See Sect. 4 and [5] for the case of dilute dipole gases. One then obtains an effective, or renormalized, activity

$$
z(\beta, \varrho)=K(\varrho) \cdot \exp [-\operatorname{const} \beta E(\varrho)]
$$

which is very small, for large $\beta$.

3. The key assertion of Theorem 2.1 is contained in (2.6)-(2.8). The number $K(\varrho)=z^{A_{0}(\varrho)} e^{S(\varrho)}$ is the "bare activity" of the multipole with charge density $\varrho$. The constant $C$ in $(2.6)$ satisfies $C \leqq 225 \cdot \ln 3$.

In order to motivate interpreting $S(\varrho)$ as an entropy we consider the example of a dipole of length $L$. In this case $\varrho(j)=\delta_{j k}-\delta_{j \ell}$, with $|k-\ell|=L, \delta_{j i}=1, i=j, \delta_{j i}=0$, 
otherwise. Then, since $A_{n}(\varrho)=2$, for $n \leqq \ln _{2} L-1$, and $A_{n}(\varrho)=1$, for $n(\varrho) \geqq n \geqq \ln _{2} L$, $A(\varrho)=(1+\alpha) \ln _{2} L+$ const, so that $S(\varrho) \leqq$ const $\ln _{2} L$.

While $K(\varrho)$ grows in $L$ like $e^{\text {const } \ln _{2} L}$ this is offset by the factor $\exp [-$ const $\beta E(\varrho)]$, where $E(\varrho) \approx(\ln L) / 2 \pi$ is the self-energy of the dipole, provided $\beta$ is large enough.

4. It turns out that the magnitude of the coefficients $c_{\mathscr{N}}$ is unimportant. Only their sign, i.e. $c_{\mathscr{N}}>0$, is crucial. If we define

$$
\lambda_{\mathscr{N}}=c_{\mathscr{N}} \frac{\left\langle\prod_{\varrho \in \mathcal{N}}(1+K(\varrho) \cos \phi(\varrho))\right\rangle_{\beta}}{\left\langle\prod_{j \in \Lambda}(1+z \cos \phi(j))\right\rangle_{\beta}},
$$

where $\langle\cdot\rangle_{\beta}$ denotes the expectation in the Gaussian measure $d \mu_{\beta}$, with mean 0 and covariance $\beta C$, we clearly have

$$
\langle\cdot\rangle(\beta, z)=\sum_{\mathscr{N} \in \mathscr{F}} \lambda_{\mathscr{N}}\langle\cdot\rangle_{\mathscr{N}}(\beta)
$$

with $0<\lambda_{\mathscr{N}}, \sum_{\mathscr{N} \in \mathscr{F}} \lambda_{\mathscr{N}}=1$.

Here $\langle\cdot\rangle_{\mathscr{N}}(\beta)$ is the state with Gibbs measure

$$
Z_{\mathscr{N}}^{-1} \prod_{\varrho \in \mathscr{N}}(1+K(\varrho) \cos \phi(\varrho)) d \mu_{\beta}(\phi) .
$$

Thus, Theorem 2.1 provides us with a representation of the state of the Coulomb gas as a convex combination of states describing dilute gases of neutral multipoles ("dipole gases").

5. As noted in Sect. 1, the factor $\left(1+K\left(\varrho_{c}\right) \cos \phi\left(\varrho_{c}\right)\right), \varrho_{c} \in \mathcal{N}, Q\left(\varrho_{c}\right) \neq 0$, can be replaced by 1 in (2.10) if $d \mu_{\beta}(\phi)$ is the infinite volume, zero-mass Gaussian measure, because the self-energy, $E\left(\varrho_{c}\right)$, of $\varrho_{c}$ is infinite if $Q\left(\varrho_{c}\right) \neq 0$. This is not true if Dirichlet boundary conditions are imposed at $\partial \Lambda$, in which case the expansion established in this section must be modified (see Appendix D).

6. The proof of Theorem 2.1 consists of an inductive construction of the ensembles $\mathscr{N}$.

\subsection{The Basic Lemma}

Lemma 2.2. Let $\mathscr{E}=\mathscr{E}_{n}$ be an n-ensemble. Then there is a family $\left\{\mathscr{E}_{\gamma}\right\}$ of $(n+1)$ ensembles, labelled by a parameter $\gamma$, such that $\mathscr{E} \rightarrow \mathscr{E} \gamma$, for all $\gamma$, and

$$
\prod_{\varrho \in \mathscr{E}}(1+K(\varrho) \cos \phi(\varrho))=\sum_{\gamma} c_{\gamma} \prod_{\varrho^{\prime} \in \mathscr{E}_{\gamma}}\left(1+K^{\prime}\left(\varrho^{\prime}\right) \cos \phi\left(\varrho^{\prime}\right)\right) .
$$

For all $\gamma, c_{\gamma}>0$, and for each $\varrho^{\prime} \in \mathscr{E}_{\gamma}$

a) $\varrho^{\prime}=\sum_{\varrho \in \mathscr{E}} \varepsilon\left(\varrho^{\prime}, \varrho\right)$, i.e. $\varrho^{\prime}$ is compatible with $\mathscr{E}$;

b) if there is a charge density $\varrho_{1}^{\prime} \subset \varrho^{\prime}$ which is compatible with $\mathscr{E}$ then

c)

$$
\begin{gathered}
\operatorname{dist}\left(\varrho_{1}^{\prime}, \varrho^{\prime}-\varrho_{1}^{\prime}\right) \leqq 2^{n+1} . \\
0<K^{\prime}\left(\varrho^{\prime}\right) \leqq e^{C_{1} A_{n}\left(\varrho^{\prime}\right)} \prod_{\varrho \in \mathscr{E}} K(\varrho)^{\left|\varepsilon\left(\varrho^{\prime}, \varrho\right)\right|} .
\end{gathered}
$$

The constant $C_{1}$ is independent of $\varrho^{\prime}$ and $n$. 
Proof. The lemma follows from iterated application of the identity

$$
\begin{aligned}
(1+ & \left.K_{\alpha} \cos \phi\left(\varrho_{\alpha}\right)\right)\left(1+K_{\beta} \cos \phi\left(\varrho_{\beta}\right)\right) \\
= & 1 / 3\left(1+3 K_{\alpha} \cos \phi\left(\varrho_{\alpha}\right)\right)+1 / 3\left(1+3 K_{\beta} \cos \phi\left(\varrho_{\beta}\right)\right) \\
& +1 / 6\left(1+3 K_{\alpha} K_{\beta} \cos \phi\left(\varrho_{\alpha}-\varrho_{\beta}\right)\right) \\
& +1 / 6\left(1+3 K_{\alpha} K_{\beta} \cos \phi\left(\varrho_{\alpha}+\varrho_{\beta}\right)\right) .
\end{aligned}
$$

Note, all densities on the right side of (2.13) are compatible with $\left\{\varrho_{\alpha}, \varrho_{\beta}\right\}$ and all coefficients are positive. Identity (2.13) is only applied to a pair $\left\{\varrho_{\alpha}, \varrho_{\beta}\right\} \subset \mathscr{E}$ if

$$
\operatorname{dist}\left(\varrho_{\alpha}, \varrho_{\beta}\right) \leqq 2^{n+1} .
$$

We start by applying (2.13) to any two factors on the left side of (2.11) corresponding to two charge densities $\varrho_{\alpha}, \varrho_{\beta}$ in $\mathscr{E}$ for which (2.14) holds. (If there are no such factors the lemma holds trivially.) The right side of identity (2.13) is then inserted on the left side of (2.11), replacing the two factors labelled by $\varrho_{\alpha}, \varrho_{\beta}$ by a sum of four terms, and by expanding we obtain a sum of four products. If one of the resulting products contains two factors labelled by densities $\varrho_{\alpha}^{\prime}, \varrho_{\beta}^{\prime}$ satisfying (2.14), we apply identity (2.13) again and expand the resulting expression into a sum of products. We repeat this operation until we obtain a sum over products indexed by ensembles $\mathscr{E}_{\gamma}$ with the property that, for arbitrary $\varrho_{1}, \varrho_{2}$ in $\mathscr{E}_{\gamma}, \operatorname{dist}\left(\varrho_{1}, \varrho_{2}\right)>2^{n+1}$.

As noted already, each application of (2.13) replaces charge densities which are compatible with $\mathscr{E}$ by other charge densities compatible with $\mathscr{E}$. Thus $\varrho^{\prime}$ $=\sum_{\varrho \in \mathscr{E}} \varepsilon\left(\varrho^{\prime}, \varrho\right) \varrho$, for all $\varrho^{\prime} \in \mathscr{E}_{\gamma}$ and all $\gamma$. Moreover, all coefficients $c_{\gamma}$ are clearly positive, [since by (2.13) each $c_{\gamma}=(1 / 3)^{n_{\gamma}}(1 / 6)^{m_{\gamma}}$, for some positive integers $n_{\gamma}$ and $m_{\gamma}$ ]. This completes the proof of (2.11) and a). Part b) of the lemma then follows directly from (2.14). Thus we are left with proving c).

At intermediate stages of our operations we have identities

$$
\prod_{\varrho \in \mathscr{E}}(1+K(\varrho) \cos \phi(\varrho))=\sum_{\mathscr{I}} c_{\mathscr{I}} \prod_{\varrho_{\alpha} \in \mathscr{I}}\left(1+K_{\alpha} \cos \phi\left(\varrho_{\alpha}\right)\right),
$$

with $\mathscr{E} \rightarrow \mathscr{I}$, (i.e. $\mathscr{E}$ is a parent of each $\mathscr{I}$ ), and $c_{\mathscr{T}}>0$, for all intermediate ensembles $\mathscr{I}$. In order to prove (2.12) we now consider some density $\varrho^{\prime} \in \mathscr{E}_{\gamma}$ and an intermediate ensemble $\mathscr{I}$ such that $\varrho^{\prime}$ is compatible with $\mathscr{I}$. (If $\varrho^{\prime}$ is not compatible with $\mathscr{I}$, further operations on the factors indexed by densities in $\mathscr{I}$ can never produce $\varrho^{\prime}$, as is immediate to see.) We must keep track of all applications of identity (2.13) necessary to produce the given $\varrho^{\prime}$, starting from $\mathscr{I}$, i.e. we must consider all possible applications of (2.13) to pairs $\left\{\varrho_{\alpha}, \varrho_{\beta}\right\} \subset \mathscr{I}$ for which either

i) $\pm \varrho_{\alpha} \subset \varrho^{\prime}, \varrho_{\beta} \cap \varrho^{\prime}=\emptyset$, or

ii) $\pm \varrho_{\beta} \subset \varrho^{\prime}, \varrho_{\alpha} \cap \varrho^{\prime}=\emptyset$, or

iii) $\pm \varrho_{\alpha} \subset \varrho^{\prime}, \pm \varrho_{\beta} \subset \varrho^{\prime}$.

In case i) the term on the right side of identity (2.13) is chosen in which $\varrho_{\beta}$ is eliminated. (Suppose not; then $\varrho_{\alpha}$ and $\varrho_{\beta}$ would have been combined to $\varrho_{\alpha} \pm \varrho_{\beta}$. At later stages, either $\varrho_{\alpha} \pm \varrho_{\beta}$ would have been eliminated, or it would have been kept, so that either $\varrho_{\alpha} \phi \varrho^{\prime}$, or $\pm \varrho_{\beta} \subset \varrho^{\prime}$.) The term in which $\varrho_{\beta}$ is eliminated is $\propto\left(1+3 K_{\alpha} \cos \phi\left(\varrho_{\alpha}\right)\right)$, so the coefficient, $3 K_{\alpha}$, of $\cos \phi\left(\varrho_{\alpha}\right)$ is independent of $K_{\beta}$ and $\varrho_{\beta}$. 
Since (2.14) has been imposed, $\varrho_{\beta}$ contains at least one density $\varrho \in \mathscr{E}$ with the property that $\operatorname{dist}\left(\varrho, \varrho^{\prime}\right) \leqq 2^{n+1}$. Thus, the total number of applications of identity (2.13) of type i) necessary to produce $\varrho^{\prime}$ is at most

$$
\operatorname{card}\left\{\varrho \in \mathscr{E}: \varrho \cap \varrho^{\prime}=\emptyset, \operatorname{dist}\left(\varrho, \varrho^{\prime}\right) \leqq 2^{n+1}\right\} .
$$

Case ii) is the same as case i) with $\alpha$ and $\beta$ interchanged.

In case iii) a term on the right side of identity (2.13) is chosen in which $\varrho_{\alpha}$ and $\varrho_{\beta}$ are combined to $\varrho_{\alpha}+\varrho_{\beta}$ or $\varrho_{\alpha}-\varrho_{\beta}$. The coefficient of $\cos \phi\left(\varrho_{\alpha} \pm \varrho_{\beta}\right)$ is $3 K_{\alpha} K_{\beta}$. Given $\varrho^{\prime}$, there are precisely card $\left\{\varrho \in \mathscr{E}: \varrho \subset \varrho^{\prime}\right\}-1$ applications of $(2.13)$ in which a term of type iii) is kept which are needed to form $\varrho^{\prime}$, independently of the order in which the constituents, $\varrho \in \mathscr{E}$, of $\varrho^{\prime}$ got paired. (The proof of this is an easy combinatorial exercise.)

From the discussion of cases i)-iii) above, we now conclude that

$$
K^{\prime}\left(\varrho^{\prime}\right) \leqq 3^{n_{\tilde{E}}\left(\varrho^{\prime}\right)} \prod_{\varrho \in \mathscr{E}} K(\varrho)^{\left|\varepsilon\left(\varrho^{\prime}, \varrho\right)\right|},
$$

where $n_{\mathscr{E}}\left(\varrho^{\prime}\right)=\operatorname{card}\left\{\varrho \in \mathscr{E}: \operatorname{dist}\left(\varrho, \varrho^{\prime}\right) \leqq 2^{n+1}\right\}$. Next, we make use of the fact that $\mathscr{E}$ was assumed to be an $n$-ensemble, i.e. for any two densities $\varrho_{1}, \varrho_{2}$ in $\mathscr{E}, \varrho_{1} \neq \varrho_{2}$, $\operatorname{dist}\left(\varrho_{1}, \varrho_{2}\right)>2^{n}$. From this property it follows immediately that a $2^{n} \times 2^{n}$ square cannot intersect more than three different charge densities in $\mathscr{E}$. Let $\bar{A}_{n}(\varrho)$ be the minimal number of $2^{n} \times 2^{n}$ squares needed to cover $\left\{j \in \Lambda\right.$ :dist $\left.(j, \operatorname{supp} \varrho) \leqq 2^{n+1}\right\}$. It is easy to check that $\bar{A}_{n}(\varrho) \leqq 25 A_{n}(\varrho)$. Thus

$$
n_{\mathscr{E}}\left(\varrho^{\prime}\right) \leqq C_{2} A_{n}\left(\varrho^{\prime}\right) \text {, }
$$

for some constant $C_{2} \leqq 75$.

From this and inequality (2.15) we obtain part c) of the lemma, with $C_{1}=C_{2} \ln 3$.

\subsection{Proof of Theorem 2.1}

The theorem follows by an induction in distance scales $2^{n}, n=0,1,2, \ldots$. Each induction step is carried out by applying Lemma 2.2 to appropriately chosen $n$-ensembles, $\mathscr{E}_{n}$. The initial ensemble is given by $\mathscr{E}_{0}=\left\{\varrho^{x}\right\}_{x \in \Lambda}, \varrho^{x}(j) \equiv \delta_{x j}$. Clearly, $\mathscr{E}_{0}$ is a $n=-1$ ensemble. By Lemma 2.2, (2.11) we have

$$
\prod_{j \in A}(1+z \cos \phi(j))=\sum_{\gamma} c_{\gamma} \prod_{\varrho \in \mathscr{E} 1, \gamma}(1+K(\varrho) \cos \phi(\varrho))
$$

where each $\mathscr{E}_{1, \gamma}$ is a 1 -ensemble. Next, we apply (2.11) again to each term in the sum on the right side of (2.16), with $\mathscr{E}=\mathscr{E}_{1, \gamma}$, for each $\gamma$. We end up with a sum over 2-ensembles. For $n \geqq n_{0}$, with $1<n_{0} \leqq \ln _{2} M$, we must however choose the ensemble $\mathscr{E}$ on the left side of identity (2.11) to be a sub-ensemble of some $\mathscr{E}_{n, \gamma}$ obtained in previous applications of (2.11), in order to avoid generating unnecessarily large charge densities and combinatorial factors, $K(\varrho)$. Roughly speaking, we shall apply the lemma to sub-ensembles which do not satisfy the assertions of Theorem 2.1. More precisely, those sub-ensembles, $\mathscr{E}_{n}=Q_{n}-\mathscr{N}_{n}$, are chosen inductively as follows : 
Let $Q_{0}=\mathscr{E}_{0}=\left\{\varrho^{x}\right\}_{x \in A}, \mathscr{N}_{0}=\emptyset$. Let $Q_{n}$ be one of the ensembles obtained after the $n^{\text {th }}$ induction step. The induction hypotheses for $Q_{n}$ are as follows:

$$
Q_{n}=\mathscr{E}_{n} \cup \mathscr{N}_{n}, \quad \mathscr{E}_{n} \cap \mathcal{N}_{n}=\emptyset
$$

$\mathscr{E}_{n}$ is an $n$-ensemble, and $\mathcal{N}_{n}$ is a maximal sub-ensemble of $Q_{n}$ defined by

a) $\mathscr{N}_{n} \supseteqq \mathscr{N}_{n-1}$, for some $\mathscr{N}_{n-1}$; a charge density $\varrho \in Q_{n}$ belongs to $\mathcal{N}_{n}$ iff

B) $\varrho$ is neutral, i.e. $Q(\varrho)=0$;

$\gamma)$ if $\varrho_{1}$ is some other, neutral charge density in $Q_{n}$,

$$
\operatorname{dist}\left(\varrho_{1}, \varrho\right) \geqq M\left[\min \left(d\left(\varrho_{1}\right), d(\varrho)\right)\right]^{\alpha} ;
$$

反)

$$
\operatorname{dist}\left(\varrho_{1}, \varrho\right) \geqq M d(\varrho)^{\alpha},
$$

for all $\varrho_{1} \in Q_{n}-\mathscr{N}_{n}$.

It is not hard to see that $\mathscr{N}_{m}=\emptyset$, for $m<n_{0}$. In order to carry out the induction step, we apply identity (2.11), Lemma 2.2 , with $\mathscr{E}=\mathscr{E}_{n}$ :

$$
\prod_{\varrho \in \mathscr{E}_{n}}(1+K(\varrho) \cos \phi(\varrho))=\sum_{\gamma} c_{\gamma} \prod_{\varrho \in \mathscr{E}_{n+1, \gamma}}\left(1+K^{\prime}\left(\varrho^{\prime}\right) \cos \phi\left(\varrho^{\prime}\right)\right) \text {. }
$$

By Lemma 2.2, a) $\mathscr{E}_{n} \rightarrow \mathscr{E}_{n+1, \gamma}$, for all $\gamma$, and each $\mathscr{E}_{n+1, \gamma}$ is an $(n+1)$-ensemble. We set $Q_{n+1} \equiv Q_{n+1, \gamma} \equiv \mathscr{E}_{n+1, \gamma} \cup \mathscr{N}_{n}$. Since $\mathscr{N}_{n} \cap \mathscr{E}_{n}=\emptyset$, and $\mathscr{E}_{n} \rightarrow \mathscr{E}_{n+1, \gamma}, \mathscr{N}_{n} \cap \mathscr{E}_{n+1, \gamma}=\emptyset$, for all $\gamma$. We now choose $\mathscr{N}_{n+1} \equiv \mathscr{N}_{n+1, \gamma}$ to consist of $\mathscr{N}_{n}$ and of a maximal subset, $\mathscr{I}_{n+1, \gamma}$, of $\mathscr{E}_{n+1, \gamma}$ in such a way that $\mathscr{I}_{n+1, \gamma} \cup \mathscr{N}_{n}$ satisfies $\alpha$ )- $\delta$ ), above (with $n$ replaced by $n+1)$. Clearly

$$
\mathscr{E}_{n+1} \equiv \mathscr{E}_{n+1, \gamma}-\mathscr{I}_{n+1, \gamma}
$$

is an $(n+1)$-ensemble, since $\mathscr{E}_{n+1} \subseteq \mathscr{E}_{n+1, \gamma}$, and $\mathscr{E}_{n+1, \gamma}$ is an $(n+1)$-ensemble, by Lemma 2.2. This completes the induction step.

Remark. One may define $\mathscr{N}_{n}$ constructively as follows:

$\left.\alpha^{\prime}\right) \mathscr{N}_{n} \supseteqq \mathscr{N}_{n-1}$, for some $\mathscr{N}_{n-1}$;

$\left.\beta^{\prime}\right)$ for each $\varrho \in Q_{n}-\mathscr{N}_{n-1}$, let $B(\varrho)$ be the set of sites within distance $<M d(\varrho)^{\alpha}$ of supp $\varrho$. Let $\mathscr{B}_{n}{ }_{n}$ be the family of $\varrho \in Q_{n}-\mathscr{N}_{n-1}$ for which $Q(\varrho)=0$ and $B(\varrho) \cap \operatorname{supp} \varrho_{1}=\emptyset$, for all $\varrho_{1} \in Q_{n}-\mathscr{N}_{n-1}, \varrho_{1} \neq \varrho$. Let $\mathscr{E}_{n}(k) \equiv Q_{n}-\left(\mathscr{N}_{n-1} \cup \mathscr{B}_{n}{ }_{n} \cup \ldots \cup \mathscr{B}_{n}^{k}\right)$ and define $\mathscr{B}_{n}^{k+1}$ to be the family of all those $\varrho \in \mathscr{E}_{n}^{(k)}$ for which $Q(\varrho)=0$ and $B(\varrho) \cap \operatorname{supp} \varrho_{1}=\emptyset$, for all $\varrho_{1} \in \mathscr{E}_{n}^{(k)}, \varrho_{1} \neq \varrho, k=1,2,3, \ldots$ We define

$$
\mathscr{N}_{n} \equiv \mathscr{N}_{n-1} \cup\left(\bigcup_{1}^{\infty} \mathscr{B}_{n}^{k}\right)
$$

For bounded $\Lambda, \mathscr{E}_{n}$ clearly converges, as $n \rightarrow \infty$, either to the empty set, or to an ensemble consisting of a single, charged element, so that Lemma 2.2 cannot be applied anymore.

Thus, by induction in $n$, we obtain identity (2.3) of Theorem 2.1, and by (2.17) and (2.18), [respectively $\left.\left.\alpha^{\prime}\right), \beta^{\prime}\right)$ ] each ensemble, $\mathscr{N}$, on the right side of (2.3) satisfies parts a) and b) of Theorem 2.1. In order to prove part c) of Theorem 2.1, let $\varrho$ be some charge density in one of the ensembles, $\mathscr{N}$, and suppose $\varrho_{1} \subset \varrho$ satisfies

$$
\operatorname{dist}\left(\varrho_{1}, \varrho-\varrho_{1}\right) \geqq R_{1} \equiv 2 M d\left(\varrho_{1}\right)^{\alpha} .
$$


In the course of building up $\varrho$ out of some collection of densities, $\left\{\varrho_{\gamma}\right\}$, with which $\varrho$ is compatible, it must have happened that some $\varrho_{\mu} \subseteq \varrho_{1}$ got paired with some $\pm \varrho_{v}$, $\varrho_{\nu} \cong \varrho-\varrho_{1}$ to form $\varrho_{\mu}+\varrho_{\nu}$. Since $\operatorname{dist}\left(\varrho_{\mu}, \varrho_{\nu}\right) \geqq R_{1}$, this happened on scale $2^{k}$ of the inductive construction, with $2^{k+1} \geqq R_{1}$. Since $R_{1} \equiv 2 M d\left(\varrho_{1}\right)^{\alpha} \geqq 2 M d\left(\varrho_{\mu}\right)^{\alpha}, \varrho_{\mu}$ is charged. For, if $\varrho_{\mu}$ is neutral, $\varrho_{\mu} \in \mathscr{N}_{k}$, as $2^{k} \geqq(1 / 2) R_{1} \geqq M d\left(\varrho_{\mu}\right)^{\alpha}$.

Suppose now that $\varrho_{\mu} \varsubsetneqq \varrho_{1}$. Then at a later induction step some $\varrho^{\prime} \supseteqq \varrho_{\mu}+\varrho_{\nu}$ got combined with some non-empty $\varrho_{\lambda} \subseteq \varrho_{1}-\varrho_{\mu}$. But

and

$$
\operatorname{dist}\left(\varrho^{\prime}, \varrho_{\lambda}\right) \leqq \operatorname{dist}\left(\varrho_{\mu}, \varrho_{\lambda}\right) \leqq d\left(\varrho_{1}\right)
$$

$$
d\left(\varrho_{1}\right)=\left(R_{1} / 2 M\right)^{\alpha^{-1}}<\left(M^{-1} 2^{k}\right)^{\alpha^{-1}}<2^{k},
$$

for $\alpha>1, M>1$. However, the expansion on scales $<2^{k}$ was already terminated at this point. Thus $\varrho_{\mu}=\varrho_{1}$, and therefore $\varrho_{1}$ is charged. This completes the proof of part c) of Theorem 2.1, (2.5).

Now we turn to the proof of (2.6)-(2.8). We choose some $\mathcal{N}$. Let $\varrho$ be some neutral density in $\mathscr{N}$, and let $n(\varrho)$ satisfy $2^{n(\varrho)} \geqq M d(\varrho)^{\alpha}$, as required in (2.8). Let $m>n(\varrho)$, and suppose $\varrho$ was produced during the induction step on scale $2^{m}$. Since, for arbitrary constituents $\varrho_{\mu}, \varrho_{\nu}$ of $\varrho$, $\operatorname{dist}\left(\varrho_{\mu}, \varrho_{\nu}\right) \leqq \mathrm{d}(\varrho)<2^{\alpha^{-1} m}<2^{m}$, for $M>1, \alpha>1$, $\varrho$ must have been paired with some $\varrho^{\prime \prime} \neq \varrho$ at distance at least $2^{m}$ from $\varrho$, and $\varrho^{\prime \prime}$ got eliminated, i.e. the first term on the right side of identity (2.13) was chosen, (with $\varrho_{\alpha}$ $=\varrho, \varrho_{\beta}=\varrho^{\prime \prime}$; see cases i), ii) in the proof of Lemma 2.2). However, since $\varrho$ is neutral, and $2^{m}>2^{n(\varrho)} \geqq M d(\varrho)^{\alpha}$ this would violate the rules for choosing $\mathscr{N}_{n}$, namely $\mathscr{N}_{n(\varrho)}$ would not have been chosen to be a maximal subset of $Q_{n(\varrho)}$. Thus, for some $m \leqq n(\varrho), \varrho \in \mathscr{N}_{m}$. By Lemma 2.2, (2.12)

$$
K(\varrho) \leqq e^{C_{1} A_{m}(\varrho)} \prod_{\varrho_{\gamma}} K\left(\varrho_{\gamma}\right)^{\left|\varepsilon\left(\varrho, \varrho_{\gamma}\right)\right|},
$$

where $\varrho=\sum \varepsilon\left(\varrho, \varrho_{\gamma}\right) \varrho_{\gamma}$, and all densities $\varrho_{\gamma}$ belong to some $(m-1)$-ensemble. Applying (2.12) again, we obtain

$$
K(\varrho) \leqq \exp \left\{C_{1}\left[A_{m}(\varrho)+\sum_{ \pm \varrho_{\gamma} \subset \varrho} A_{m-1}\left(\varrho_{\gamma}\right)\right]\right\} \prod_{ \pm \varrho_{\sigma} \subset \varrho} K\left(\varrho_{\sigma}\right)
$$

for densities $\varrho_{\sigma}$ in some $(m-2)$-ensemble.

Next, we make use of the inequality: If $\operatorname{dist}\left(\varrho_{\gamma}, \varrho_{\gamma^{\prime}}\right)>2^{k}$, for all $\gamma \neq \gamma^{\prime}$ then

$$
\sum_{\varrho_{\gamma} \subset \varrho} A_{k}\left(\varrho_{\gamma}\right) \leqq C_{3} A_{k}(\varrho),
$$

for some constant $C_{3} \leqq 3$. (The proof is straightforward; see also end of proof of Lemma 2.2.) By combining (2.21) and (2.22) and a recursion, (2.6) and (2.7) follow, with $C \leqq C_{1} C_{3} \leqq 225 \ln 3$; (the upper bound on $C$ is vastly larger than what one could presumably obtain by more detailed, combinatorial arguments). This completes the proof of Theorem 2.1 .

\subsection{The Fractional Charge Observable}

Next, we make a minor modification in the above arguments to include, in the inductive construction of the ensembles, $\mathcal{N}$, an observable

$$
A(\phi)=\cos \phi\left(\xi \varrho_{0}\right),
$$


where $\varrho_{0}$ is some charge density of compact support, and $\xi \in(0,1)$. These modifications are not needed for the proof of Theorems A and C. As an example we mention

$$
\varrho_{0}=\delta_{j 0}-\delta_{j x}, \quad|x|=L,
$$

so that $\xi_{Q_{0}}, \xi \neq 0,1$, is the charge density of a dipole consisting of two fractional charges, $\pm \xi$, separated by a distance $L$.

We wish to derive an expansion for $\cos \phi\left(\xi \varrho_{0}\right) \prod_{j \in A}(1+z \cos \phi(j))$ for which the analogue of Theorem 2.1 holds. First, we note the identity

$$
\begin{aligned}
& \cos \phi\left(\xi \varrho_{0}\right) \prod_{j \in \operatorname{supp} \varrho_{0}}(1+z \cos \phi(j)) \\
& =\sum_{\alpha=1}^{n_{0}} c_{\alpha}\left(\cos \phi\left(\xi \varrho_{0}\right)+z_{\alpha} \cos \phi\left(\xi \varrho_{0}+\varrho_{\alpha}\right)\right),
\end{aligned}
$$

with $c_{\alpha}>0$, for all $\alpha$, and $\sum_{\alpha=1}^{n_{0}} c_{\alpha}=1 ; \varrho_{\alpha}(j)=0$, \pm 1 , for $j \in \operatorname{supp} \varrho_{0}$, and $\varrho_{\alpha}(j)=0$, for $j \notin \operatorname{supp} \varrho_{0} ; 0<z_{\alpha}<\infty$, with $z_{\alpha} \propto z^{A_{0}\left(\varrho_{\alpha}\right)}$.

Identity (2.24) follows by induction from the identities

$$
\begin{aligned}
\cos \phi & \left(\xi \varrho_{0}\right)\left(1+K_{1} \cos \phi\left(\varrho_{1}\right)\right) \\
= & 1 / 2\left(\cos \phi\left(\xi \varrho_{0}\right)+K_{1} \cos \phi\left(\xi \varrho_{0}+\varrho_{1}\right)\right) \\
& +1 / 2\left(\cos \phi\left(\xi \varrho_{0}\right)+K_{1} \cos \phi\left(\xi \varrho_{0}-\varrho_{1}\right)\right),
\end{aligned}
$$

and

$$
\begin{aligned}
(\cos \phi & \left.\left(\xi \varrho_{0}\right)+K_{1} \cos \phi\left(\varrho_{1}\right)\right)\left(1+K_{2} \cos \phi\left(\varrho_{2}\right)\right) \\
= & 1 / 3\left(\cos \phi\left(\xi \varrho_{0}\right)+3 K_{1} \cos \phi\left(\varrho_{1}\right)\right) \\
& +1 / 6\left(\cos \phi\left(\xi \varrho_{0}\right)+3 K_{2} \cos \phi\left(\xi \varrho_{0}+\varrho_{2}\right)\right) \\
& +1 / 6\left(\cos \phi\left(\xi \varrho_{0}\right)+3 K_{2} \cos \phi\left(\xi \varrho_{0}-\varrho_{2}\right)\right) \\
& +1 / 6\left(\cos \phi\left(\xi \varrho_{0}\right)+3 K_{1} K_{2} \cos \phi\left(\varrho_{1}+\varrho_{2}\right)\right) \\
& +1 / 6\left(\cos \phi\left(\xi \varrho_{0}\right)+3 K_{1} K_{2} \cos \phi\left(\varrho_{1}-\varrho_{2}\right)\right),
\end{aligned}
$$

with $\varrho_{1}=\xi \varrho_{0}+\varrho_{1}^{\prime}$, in (2.26). Identities (2.13) and (2.26) are our basic tools to expand each term in the sum

$$
\sum_{\alpha=1}^{n_{0}} c_{\alpha}\left(\cos \phi\left(\xi \varrho_{0}\right)+z_{\alpha} \cos \phi\left(\xi \varrho_{0}+\varrho_{\alpha}\right)\right) \prod_{j \in \Lambda \backslash \text { supp } \varrho_{0}}(1+z \cos \phi(j))
$$

into a sum of terms, as in Theorem 2.1, (2.3).

In order to estimate the terms produced by that expansion, (Sects. 3 and 4) we will need the following condition on $\varrho_{0}$.

Condition (2.28). If $\varrho_{0}$ can be decomposed as

$$
\varrho_{0}=\varrho^{\prime}+\left(\varrho_{0}-\varrho^{\prime}\right), \text { with } \varrho^{\prime} \neq \emptyset \neq \varrho_{0}-\varrho^{\prime},
$$

and $\varrho^{\prime}$ satisfies $\operatorname{dist}\left(\varrho^{\prime}, \varrho_{0}-\varrho^{\prime}\right) \geqq 2 M d\left(\varrho^{\prime}\right)^{\alpha}$, then $Q\left(\xi \varrho^{\prime}\right) \in\left[m+\xi^{\prime}, m+1-\xi^{\prime}\right]$, for some integer $m, 0<\xi^{\prime} \leqq 1 / 2$, i.e. $Q\left(\xi \varrho^{\prime}\right)$ is fractional. 
In the example of the dipole, $\varrho^{\prime}=\delta_{x j}, Q\left(\xi \varrho^{\prime}\right)=\xi$, so Condition (2.28) is fulfilled if $0<\xi<1$, i.e. the charges in the dipole are fractional. We now prove

Theorem 2.3. There exists a family, $\mathscr{F}_{\Lambda}\left(\varrho_{0}\right)$, of ensembles, $\mathscr{N}$, such that

$$
\begin{aligned}
& \cos \phi\left(\xi \varrho_{0}\right) \prod_{j \in \Lambda}(1+z \cos \phi(j)) \\
& \quad=\sum_{\mathscr{N} \in \mathscr{F}_{\Lambda}\left(\varrho_{0}\right)} c_{\mathscr{N}}\left(\cos \phi\left(\xi \varrho_{0}\right)+K\left(\varrho^{\mathcal{N}}\right) \cos \phi\left(\xi \varrho_{0}+\varrho^{\mathscr{N}}\right)\right) \cdot \prod_{\varrho \in \mathcal{N}}(1+K(\varrho) \cos \phi(\varrho)),
\end{aligned}
$$

where each $\mathscr{N}$ satisfies properties a)-c) stated in Theorem 2.1, $\mathscr{N} \cup\left\{\xi \varrho_{0}\right\}$ and $\mathscr{N} \cup\left\{\xi \varrho_{0}+\varrho^{\mathcal{N}}\right\}$ satisfy a) and b), and if Condition (2.28) is imposed on $\varrho_{0}$ then c) holds, too, in particular, if $\varrho_{1} \subset \xi \varrho_{0}+\varrho^{\mathscr{N}}$ is such that $\operatorname{dist}\left(\varrho_{1}, \xi \varrho_{0}+\varrho^{\mathscr{N}}-\varrho_{1}\right)$ $\geqq 2 M d\left(\varrho_{1}\right)^{\alpha}$ then $\varrho_{1}$ is charged, with $Q\left(\varrho_{1}\right) \in\left[m+\xi^{\prime}, m+1-\xi^{\prime}\right]$, for some integer $m$. The coefficients $K(\varrho), \varrho \in \mathscr{N}$, satisfy (2.6)-(2.8), and

$$
K\left(\varrho^{\mathcal{N}}\right) \leqq k^{A_{0}\left(\varrho_{0}\right)} z^{A_{0}\left(\varrho^{\mathscr{N}}\right)} e^{C \cdot A\left(s\left(\varrho_{0}, \varrho^{\mathscr{N}}\right)\right)}
$$

where $k$ is some constant, $s\left(\varrho_{0}, \varrho^{\mathcal{N}}\right) \equiv \operatorname{supp} \varrho_{0} \cup \operatorname{supp} \varrho^{\mathcal{N}}$, and $C$ and $A$ are as in (2.6)-(2.8). Moreover, the coefficients $c_{\mathscr{N}}, K\left(\varrho^{\mathcal{N}}\right), K(\varrho), \varrho \in \mathscr{N}$, are independent of $\xi$.

Remark. It is not hard to also prove a variant of Theorem 2.3 with $\cos \phi\left(\xi \varrho_{0}\right)$ replaced by $\prod_{j=1}^{n} \cos \phi\left(\xi \varrho_{j}\right)$, where each $\varrho_{j}$ satisfies Condition (2.28). The proof of this is an easy generalization of the one given below.

Proof of Theorem 2.3. We closely follow the proof of Theorem 2.1. In order to do the induction step we need a slight generalization of Lemma 2.2: We consider $n$-ensembles of the form

$$
\mathscr{E}=\tilde{\mathscr{E}} \cup\{\tilde{\varrho}\},
$$

corresponding to a product

$$
\left(\cos \phi\left(\xi \varrho_{0}\right)+K(\tilde{\varrho}) \cos \phi\left(\xi \varrho_{0}+\tilde{\varrho}\right)\right) \prod_{\varrho \in \tilde{\sigma}}(1+K(\varrho) \cos \phi(\varrho)) .
$$

The definition of an $n$-ensemble is as before, but it is required that

$$
\operatorname{dist}\left(s\left(\varrho_{0}, \tilde{\varrho}\right), \varrho\right)>2^{n}, \quad \text { for all } \varrho \in \tilde{\mathscr{E}}
$$

with

$$
s\left(\varrho_{0}, \tilde{\varrho}\right)=\operatorname{supp} \varrho_{0} \cup \operatorname{supp} \tilde{\varrho} .
$$

In the pairing step of the proof of Lemma 2.2, see (2.14), we treat the factor $\cos \phi\left(\xi \varrho_{0}\right)+K(\tilde{\varrho}) \cos \phi\left(\xi \varrho_{0}+\varrho\right)$ as if it were $=1+K(\varrho) \cos \phi(\varrho)$, where $\hat{\varrho}$ is a fake charge density with supp $\hat{\varrho}=s\left(\varrho_{0}, \tilde{\varrho}\right), d(\hat{\varrho})=\operatorname{diam}\left(s\left(\varrho_{0}, \tilde{\varrho}\right)\right), Q(\hat{\varrho})=Q(\tilde{\varrho})$ and $K(\hat{\varrho})$ $=K(\tilde{\varrho})$. Thus, the factor $\left(\cos \phi\left(\xi \varrho_{0}\right)+K(\tilde{\varrho}) \cos \phi\left(\xi \varrho_{0}+\tilde{\varrho}\right)\right)$ may be paired with a factor $(1+K(\varrho) \cos \phi(\varrho))$ iff

$$
\operatorname{dist}(\hat{\varrho}, \varrho) \leqq 2^{n+1} \text {. }
$$

If this condition is verified, the product

$$
\left(\cos \phi\left(\xi \varrho_{0}\right)+K(\tilde{\varrho}) \cos \phi\left(\xi \varrho_{0}+\varrho\right)\right)(1+K(\varrho) \cos \phi(\varrho))
$$


is replaced by the right side of identity (2.26), which is inserted in (2.31) and expanded, etc. The combinatorial aspects of identity (2.26), in particular the multiplication of the coefficients $K_{1}, K_{2}$ and $K_{1} K_{2}$ by factors of 3, are identical to the ones of identity (2.13). Thus, the identity

$$
\begin{aligned}
& \left(\cos \phi\left(\xi \varrho_{0}\right)+K(\varrho) \cos \phi\left(\xi \varrho_{0}+\varrho\right)\right) \prod_{\varrho \in \tilde{\tilde{E}}}(1+K(\varrho) \cos \phi(\varrho)) \\
& \quad=\sum_{\gamma} c_{\gamma}\left(\cos \phi\left(\xi \varrho_{0}\right)+K^{\prime}\left(\varrho^{\prime}\right) \cos \phi\left(\xi \varrho_{0}+\tilde{\varrho}^{\prime}\right)\right) \cdot \prod_{\varrho^{\prime} \in \tilde{E}_{\gamma}}\left(1+K^{\prime}\left(\varrho^{\prime}\right) \cos \phi\left(\varrho^{\prime}\right)\right)
\end{aligned}
$$

replaces (2.11), and parts a)-c) of Lemma 2.2 hold, with $\mathscr{E}_{\gamma}$ replaced by $\tilde{\mathscr{E}}_{\gamma} \cup\left\{\tilde{\varrho}^{\prime}\right\}$. In particular, $\tilde{\mathscr{E}}_{\gamma}$ is an $(n+1)$-ensemble, and

for all $\varrho^{\prime} \in \tilde{\mathscr{E}}_{\gamma}$, and all $\gamma$.

$$
\operatorname{dist}\left(s\left(\varrho_{0}, \tilde{\varrho}^{\prime}\right), \varrho^{\prime}\right)>2^{n+1},
$$

With the above modifications, the proof of Theorem 2.3 is virtually identical to the one of Theorem 2.1, in particular, the above generalization of Lemma 2.2 permits us to carry out the induction steps. The initial ensembles are

$$
\mathscr{E}_{0}^{\mathscr{O}(\alpha)}=\left\{\varrho_{x}\right\}_{x \in \Lambda \backslash \text { supp } \varrho_{0}} \cup\left\{\varrho_{\alpha}\right\},
$$

where $\varrho_{\alpha}$ is one of the charge densities appearing on the right side of (2.24). Inequality (2.30) is proven in the same way as (2.6)-(2.8) if one uses the simple fact that $z_{\alpha} \leqq k^{A_{0}\left(\varrho_{0}\right)} z^{A_{0}\left(\varrho_{\alpha}\right)}$; see (2.24). The last point to be checked is that part c) of Theorem 2.1 holds in the present situation, provided Condition (2.28) is imposed: If $\varrho \in \mathscr{N}$ the proof of c) is as before. If $\varrho=\xi \varrho_{0}$ Condition (2.28) just says that c) holds. Hence we must only consider the case $\varrho=\xi \varrho_{0}+\varrho^{\mathscr{N}}$. Let $\varrho_{1} \subset \varrho$ be such that $\operatorname{dist}\left(\varrho_{1}, \varrho-\varrho_{1}\right) \geqq 2 M d\left(\varrho_{1}\right)^{\alpha}$. If $\varrho_{1} \cap \operatorname{supp} \varrho_{0} \neq \emptyset$ then $Q\left(\varrho_{1}\right) \neq 0$, since, by Condition

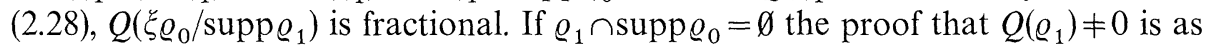
before.

\section{Corollary 2.4.}

$\prod_{j \in \Lambda}(1+z \cos \phi(j))=\sum_{\mathscr{N} \in \mathscr{F}_{\Lambda}\left(\varrho_{0}\right)} c_{\mathcal{N}}\left(1+K\left(\varrho^{\mathcal{N}}\right) \cos \phi\left(\varrho^{\mathscr{N}}\right)\right) \cdot \prod_{\varrho \in \mathcal{N}}(1+K(\varrho) \cos \phi(\varrho))$,

where $\mathscr{F}_{\Lambda}\left(\varrho_{0}\right), c_{\mathscr{N}}, K\left(\varrho^{\mathscr{N}}\right)$ and $K(\varrho)$ are as in Theorem 2.3 .

Proof. Follows from Theorem 2.3, in particular the fact that $\mathscr{F}_{\Lambda}\left(\varrho_{0}\right), c_{\mathscr{N}}, K\left(\varrho^{\mathscr{N}}\right)$, and $K(\varrho)$ are independent of $\xi$, by letting $\xi$ tend to 0 .

\section{Charged Constituents and Bounds on the Effective Activity of Charge Densities}

In this section we estimate the total number of isolated, charged constituents of a given charge density $\varrho$ in one of the ensembles $\mathcal{N}$ constructed in Theorems $2.1-2.3$ in terms of its entropy. In order to accomplish this, we use (2.5), part c) of Theorems 2.1 and 2.3. We show that sufficiently many constituents $\varrho_{1}$ of $\varrho$ are far from $\varrho-\varrho_{1}$, in the sense of inequality (2.5), and hence are charged, so as to permit us to renormalize the bare activity $K(\varrho)$ by electrostatic inequalities, see Sect. 4 , in such a way that if $\beta$ is large enough the renormalized activity is bounded above by 1 . 
More precisely, we shall show in Sect. 4 that the renormalized activity, $z(\beta, \varrho)$, of some $\varrho \in \mathscr{N}$ is bounded by

$$
\begin{aligned}
z(\beta, \varrho) & \leqq K(\varrho) \exp \left[-\beta E_{1 \mathrm{co}}(\varrho, \mathscr{N})\right] \\
& \leqq \exp [(c-\beta d) A(\varrho)]
\end{aligned}
$$

for some positive, finite constants, $c, d$. Here $E_{\text {loc }}(\varrho, \mathscr{N})$ is a (local) portion of the electrostatic self-energy of $\varrho$ in the ensemble $\mathscr{N}$. Clearly, inequality (3.1) requires proving a lower bound on $E_{\text {loc }}(\varrho, \mathscr{N})$ in terms of $A(\varrho)$. We state this lower bound below and then prove a combinatorial lemma needed to establish-it. We now need some definitions:

Let $\mathscr{S}_{k}(\varrho)$ be a minimal collection of $2^{k} \times 2^{k}$ squares covering the support of $\varrho$. By minimal we mean that $\mathscr{S}_{k}(\varrho)$ is chosen such that its cardinality, $\left|\mathscr{S}_{k}(\varrho)\right|$, is minimal, i.e.

$$
\left|\mathscr{S}_{k}(\varrho)\right|=A_{k}(\varrho),
$$

(the area of supp $\varrho$ on scale $2^{k}$ ).

Now, we define $\mathscr{S}_{k}^{\prime}(\varrho)$ to be the sub-collection of those squares $s^{\prime}$ in $\mathscr{S}_{k}(\varrho)$ which are far separated from other squares in $\mathscr{S}_{k}(\varrho)$, in the sense that

$$
\operatorname{dist}\left(s^{\prime}, s\right) \geqq 2 M 2^{\alpha k} \equiv 2^{\alpha k+b},
$$

for all $s \in \mathscr{S}_{k}(\varrho), s \neq s^{\prime}$. [If $\mathscr{S}_{k}(\varrho)$ consists of only one square and $Q(\varrho)=0$ we set $\mathscr{S}_{k}^{\prime}(\varrho)=\emptyset$. $]$ Here we have set $2 M \equiv 2^{b}$, for some $b>1$. We define $\mathscr{S}_{k}^{\prime \prime}(\varrho)=\mathscr{S}_{k}(\varrho) \backslash \mathscr{S}_{k}^{\prime}(\varrho)$ and set $\mathscr{S}_{0}(\varrho)=\mathscr{S}_{0}^{\prime}(\varrho)$, so that $\left|\mathscr{S}_{0}^{\prime}(\varrho)\right|=A_{0}(\varrho)$. We note that, by $(2.5)$, respectively Theorem $2.3, \mathrm{c}), s^{\prime} \cap \varrho$ is charged, for each $s^{\prime} \in \mathscr{S}_{k}^{\prime}(\varrho), k=0,1,2, \ldots$ Let

$$
A^{\prime}(\varrho)=\sum_{k=1}^{\infty}\left|\mathscr{S}_{k}^{\prime}(\varrho)\right|+A_{0}(\varrho)
$$

Note that, for $k>\ln _{2} d(\varrho), \mathscr{S}_{k}(\varrho)$ consists of a single square covering all of supp $\varrho$. Hence $\mathscr{S}_{k}^{\prime}(\varrho)=\emptyset,\left|\mathscr{S}_{k}^{\prime}(\varrho)\right|=0$, so that the sum on the right side of (3.4) terminates at some $k=k(\varrho) \leqq \ln _{2} d(\varrho)$ [unless $\varrho$ is charged, in which case $k(\varrho)=\infty,\left|\mathscr{S}_{k}^{\prime}(\varrho)\right|=1$, for all $\left.k>\ln _{2} d(\varrho)\right]$. In Sect. 4 we prove that each constituent of a charge density $\varrho \in \mathscr{N}$ covered by some square $s^{\prime} \in \mathscr{S}_{k}^{\prime}(\varrho), k=0,1,2, \ldots$, contributes at least a positive constant $D_{1}$, independent of $\varrho$ and $\mathscr{N}$, to the electrostatic self-energy $E_{\text {loc }}(\varrho, \mathscr{N})$. Thus

$$
E_{\mathrm{loc}}(\varrho, \mathscr{N}) \geqq D_{1} A^{\prime}(\varrho)
$$

The main result of this section is

Lemma 3.1.

$$
A(\varrho) \leqq D_{2} A^{\prime}(\varrho),
$$

for some finite constant $D_{2}$ independent of $\varrho$ and $\mathscr{N}$.

Remark. By combining inequalities (3.5) and (3.6) we conclude

$$
E_{\text {loc }}(\varrho, \mathscr{N}) \geqq\left(D_{1} / D_{2}\right) A(\varrho),
$$


and, using Theorem 2.1,(2.6)-(2.8), we obtain

$$
\begin{aligned}
z(\beta, \varrho) \leqq & K(\varrho) e^{-\beta E_{1 \mathrm{oc}}(\varrho)} \\
\leqq & \exp \left[\left(C+\ln z-\beta D_{1} / D_{2}\right) A_{0}(\varrho)\right] \\
& \cdot \exp \left[\left(C-\beta D_{1} / D_{2}\right)\left(A(\varrho)-A_{0}(\varrho)\right)\right] .
\end{aligned}
$$

For $z \leqq$ const $e^{\varepsilon \beta}, \varepsilon<D_{1} / D_{2}$, this yields (3.1). A similar estimate follows from Theorem 2.3, (2.30).

Proof of Lemma 3.1. We define a function $\gamma$ by

$$
\gamma(k)=\left[\alpha^{-1}(k-b-2)\right]
$$

where $[x]$ denotes the integer part of $x$.

We now fix some $k$, with $2^{\gamma(k)}<d(\varrho)$, so that $\mathscr{S}_{\gamma(k)}(\varrho)$ contains at least two squares. Let $s_{1}$ be some square in $\mathscr{S}_{\gamma(k)}^{\prime \prime}(\varrho)$. [It is assumed, here, that $\mathscr{S}_{\gamma(k)}^{\prime \prime}(\varrho) \neq \emptyset$. Hence $\left|\mathscr{S}_{\gamma(k)}^{\prime \prime}(\varrho)\right| \geqq 2$.] Then, by the definition of $\mathscr{S}_{\gamma(k)}^{\prime \prime}(\varrho)$, there exists some square $s_{2} \in \mathscr{S}_{\gamma(k)}^{\prime \prime}(\varrho)$ such that

$$
\operatorname{dist}\left(s_{1}, s_{2}\right)<2 M 2^{\alpha \gamma(k)} \leqq 2^{b} 2^{k-b-2}=2^{k-2} .
$$

Since $d\left(s_{1}\right)=d\left(s_{2}\right)=\sqrt{2} 2^{\gamma(k)}$,

$$
d\left(s_{1}\right)+d\left(s_{2}\right)+\operatorname{dist}\left(s_{1}, s_{2}\right) \leqq 2^{\gamma(k)+2}+2^{k-2}<2^{k-1},
$$

provided $0 \leqq \gamma(k) \leqq k-4$ which holds for $b>1, \alpha>1$. Thus, $s_{1}$ and $s_{2}$ can be covered by a single square $s \in \mathscr{S}_{k}(\varrho)$. Next, let $s_{1}, \ldots, s_{m}, m \geqq 3$, be squares in $\mathscr{S}_{\gamma(k)}^{\prime \prime}(\varrho)$ such that

$$
\operatorname{dist}\left(s_{\ell}, s_{\ell+1}\right)<2 M 2^{\alpha \gamma(k)}, \quad \ell=1, \ldots, m-1 .
$$

Then, since

$$
d\left(s_{\ell-1}\right)+d\left(s_{\ell}\right)+d\left(s_{\ell+1}\right)+2\left(2 M 2^{\alpha \gamma(k)}\right)<2^{\gamma(k)+3}+2^{k-1}<2^{k},
$$

$s_{\ell-1}, s_{\ell}$ and $s_{\ell+1}$ can be covered by a single $2^{k} \times 2^{k}$ square, for any $\ell=2,3, \ldots, m-1$. Therefore $\left[\frac{\mathrm{m}}{2}\right] \leqq \frac{\mathrm{m}}{2} 2^{k} \times 2^{k}$ squares suffice to cover $s_{1}, \ldots, s_{m}$. for any $m=2,3, \ldots$. From this we easily derive the inequality

$$
\begin{aligned}
A_{k}(\varrho) & =\left|\mathscr{S}_{k}(\varrho)\right| \leqq \frac{1}{2}\left|\mathscr{S}_{\gamma(k)}^{\prime \prime}(\varrho)\right|+\left|\mathscr{S}_{\gamma(k)}^{\prime}(\varrho)\right| \\
& \leqq \frac{1}{2} A_{\gamma(k)}(\varrho)+\left|\mathscr{S}_{\gamma(k)}^{\prime}(\varrho)\right|,
\end{aligned}
$$

provided $\mathscr{S}_{\gamma(k)}(\varrho)$ contains at least two squares. We recall that

$$
A(\varrho)=\sum_{n=0}^{n(\varrho)} A_{n}(\varrho),
$$

with

$$
n(\varrho) \leqq\left[\ln _{2}\left(M d(\varrho)^{\alpha}\right)\right]+1
$$

see Theorem 2.1. We therefore need to prove (3.8) only for $k \leqq n(\varrho)$. For such values of $k, 2^{\gamma(k)}<d(\varrho)$, (provided $\alpha<2$ ). Therefore $\left|\mathscr{S}_{\gamma(k)}(\varrho)\right| \geqq 2$, and the above proof of (3.8) applies. [For $k>n(\varrho),(3.8)$ would, however, be false.] 
Let $\delta \equiv b-2$. Clearly, inequality (3.8) can only be applied if

$$
\gamma(k)=\left[\alpha^{-1}(k-\delta)\right] \geqq 0, \quad \text { i.e. } \quad k \geqq \delta .
$$

For each $k$, we now iterate inequality (3.8) $\ell=\ell(k)$ times, where $\ell(k)$ is the maximal number for which

$$
\gamma^{\ell(k)}(k) \geqq 0, \quad \text { i.e. } \quad \gamma^{\ell(k)-1}(k) \geqq \delta .
$$

Here $\gamma^{m}$ denotes the $m$-fold composition of $\gamma$ with itself. This yields

$$
\begin{aligned}
A_{k}(\varrho) & \leqq \sum_{m=0}^{\ell-1} 2^{-m}\left|\mathscr{S}_{\gamma^{m+1}(k)}^{\prime}(\varrho)\right|+2^{-\ell} A_{\gamma^{\ell}(k)}(\varrho) \\
& \leqq \sum_{m=0}^{\ell-1} 2^{-m}\left|\mathscr{S}_{\gamma^{m+1}(k)}^{\prime}(\varrho)\right|+2^{-\ell} A_{0}(\varrho)
\end{aligned}
$$

with $\ell=\ell(k)$ given by (3.9). We have used that

$$
A_{0}(\varrho) \geqq A_{j}(\varrho), \text { for all } j \geqq 0 .
$$

We now estimate $\ell(k)$. For this purpose we extend the definition of $\gamma$ to the whole interval $[\delta, \infty)$, by setting $\gamma(x)=\left[\alpha^{-1}(x-\delta)\right]$, for arbitrary $x \geqq \delta$. Obviously, $\gamma$ is monotone increasing, and

$$
\gamma(x)>\alpha^{-1}(x-\delta)-1
$$

These two properties yield

$$
\gamma^{2}(x)=\gamma(\gamma(x)) \geqq \gamma\left(\alpha^{-1}(x-\delta)-1\right)>\alpha^{-2} x-\alpha^{-2} \delta-\alpha^{-1} \delta-\alpha^{-1}-1,
$$

and, by induction,

$$
\gamma^{m}(x)>\alpha^{-m} x-\delta\left(\sum_{j=1}^{m} \alpha^{-j}\right)-\sum_{j=0}^{m-1} \alpha^{-j} .
$$

If we let $m \rightarrow \infty$ in the last two terms on the right side of (3.12) we obtain

$$
\gamma^{m}(x)>\alpha^{-m} x-(\alpha-1)^{-1}(\alpha+\delta) .
$$

For $k \geqq(\alpha-1)^{-1}(\alpha+\delta)$, we define $\ell_{0}(k)$ to be the maximal integer for which

$$
\alpha^{-\ell_{0}(k)} k-(\alpha-1)^{-1}(\alpha+\delta) \geqq 0 \text {. }
$$

Then $\gamma^{\ell_{0}(k)}(k)>0$ and therefore $\ell(k) \geqq \ell_{0}(k)$.

By taking logarithms in (3.13) we thus obtain

$$
\ell(k) \geqq \begin{cases}0, & 0 \leqq k<(\alpha-1)^{-1}(\alpha+\delta) \\ {\left[\left(1 / \ln _{2} \alpha\right) \ln _{2}\left(k / k_{0}\right)\right],} & \text { otherwise, }\end{cases}
$$

where $k_{0}=(\alpha-1)^{-1}(\alpha+\delta)$.

Next, we estimate the cardinality, $\left|N_{m, j}\right|$, of the sets

$$
N_{m, j} \equiv\left\{k: \gamma^{m}(k)=j\right\},
$$

for given $m$ and $j$. By the definition of $\gamma$

$$
a^{-1}(x-\delta)-1 \leqq \gamma(x) \leqq \alpha^{-1}(x-\delta) .
$$


Since $\gamma$ is monotone increasing,

$$
\begin{aligned}
\alpha^{-2} x-\alpha^{-2} \delta-\alpha^{-1} \delta-\alpha^{-1}-1 & \leqq \gamma\left(\alpha^{-1}(x-\delta)-1\right) \leqq \gamma(\gamma(x)) \leqq \gamma\left(\alpha^{-1}(x-\delta)\right) \\
& \leqq \alpha^{-2} x-\alpha^{-2} \delta-\alpha^{-1} \delta
\end{aligned}
$$

and, by induction,

$$
\alpha^{-m} x-\delta\left(\sum_{\ell=1}^{m} \alpha^{-\ell}\right)-\sum_{\ell=0}^{m-1} \alpha^{-\ell} \leqq \gamma^{m}(x) \leqq \alpha^{-m} x-\delta\left(\sum_{\ell=1}^{m} \alpha^{-\ell}\right) .
$$

Next, let $k_{-}$be the minimal and $k_{+}$the maximal integer in $N_{j, m}$. Then

Thus

$$
\alpha^{-m} k_{+}-\delta\left(\sum_{1}^{m} \alpha^{-\ell}\right)-\sum_{0}^{m-1} \alpha^{-\ell} \leqq \gamma^{m}\left(k_{+}\right)=j=\gamma^{m}\left(k_{-}\right) \leqq \alpha^{-m} k_{-}-\delta\left(\sum_{1}^{m} \alpha^{-\ell}\right) .
$$

$$
\left|N_{m, j}\right|=k_{+}-k_{-}+1<\alpha^{m}\left(\frac{2 \alpha}{\alpha-1}\right), \quad \text { for } \quad \alpha>1
$$

Using (3.10) we have

$$
\begin{aligned}
A(\varrho) & =\sum_{k=0}^{n(\varrho)} A_{k}(\varrho) \\
& \leqq \sum_{k=0}^{n(\varrho)}\left\{2^{-\ell(k)} A_{0}(\varrho)+\sum_{m=0}^{\ell(k)-1} 2^{-m}\left|\mathscr{S}_{\gamma^{m+1}(k)}^{\prime}(\varrho)\right|\right\} \\
& \leqq E A_{0}(\varrho)+F \sum_{j=0}^{k(\varrho)}\left|\mathscr{S}_{j}^{\prime}(\varrho)\right|
\end{aligned}
$$

where

$$
E \leqq \sum_{k=0}^{\infty} 2^{-\ell(k)}
$$

By (3.14) and (3.18)

with

$$
\left.\begin{array}{c}
E \leqq k_{0}+\sum_{k=k_{0}+1}^{\infty}\left(k_{0} / k\right)^{1 / \ln _{2} \alpha}, \\
k_{0}=(\alpha-1)^{-1}(\alpha+\delta) .
\end{array}\right\}
$$

Therefore $E$ is finite, provided

$$
1<\alpha<2, \text { i.e. } 1 / \ln _{2} \alpha>1 .
$$

Now we turn to our estimate of $F$. We use

$$
\begin{aligned}
& \sum_{k=0}^{n(\varrho)} \sum_{m=0}^{\ell(k)-1} 2^{-m}\left|\mathscr{S}_{\gamma^{m+1}(k)}^{\prime}(\varrho)\right| \\
& \quad \sum_{j=0}^{k(\varrho)}\left(\sum_{k=0}^{n(\varrho)} \sum_{m=0}^{\ell(k)-1} 2^{-m} \delta_{\gamma^{m+1}(k), j}\right)\left|\mathscr{S}_{j}^{\prime}(\varrho)\right| \\
& \quad \sum_{j=0}^{k(\varrho)}\left(\sum_{m, k=0}^{\infty} 2^{-m} \delta_{\gamma^{m+1}(k), j}\right)\left|\mathscr{S}_{j}^{\prime}(\varrho)\right| \\
& =\sum_{j=0}^{k(\varrho)}\left(\sum_{m=0}^{\infty} 2^{-m}\left|N_{j, m}\right|\right)\left|\mathscr{S}_{j}^{\prime}(\varrho)\right| \\
& \leqq 4 \alpha(\alpha-1)^{-1}(1-\alpha / 2)^{-1} \sum_{j=0}^{k(\varrho)}\left|\mathscr{S}_{j}^{\prime}(\varrho)\right|
\end{aligned}
$$


where we have used the definition of $N_{j, m}$, inequality (3.16) and condition (3.20). Hence

$$
F \leqq 8 \alpha\left(3 \alpha-\alpha^{2}-2\right)^{-1} \text {. }
$$

Lemma 3.1 now follows from (3.17), (3.19), and (3.21), by setting $D_{2}=\max (E, F)$.

Remark. Inequality (3.17) is a somewhat finer version of Lemma 3.1 which can be used to slightly improve the upper bound (3.7) on $z(\beta, \varrho)$.

\section{Electrostatics and the Renormalization of Multipole Densities and Activities}

One of the main issues of this section is to interpret and prove inequalities (3.1) and (3.5), i.e.

$$
\left.\begin{array}{l}
z(\beta, \varrho) \leqq K(\varrho) \exp \left[-\beta E_{1 \mathrm{loc}}(\varrho, \mathscr{N})\right], \\
E_{\text {loc }}(\varrho, \mathscr{N}) \geqq D_{1} A^{\prime}(\varrho),
\end{array}\right\}
$$

for some positive constant $D_{1}$. Here $z(\beta, \varrho)$ is the renormalized, or effective activity of a (neutral) multipole with charge density $\varrho$, and $E_{10 \mathrm{c}}(\varrho, \mathscr{N})$ is a "local" portion of the electrostatic self-energy of $\varrho$. Moreover, $\mathscr{N}$ is a multipole ensemble satisfying the properties stated in Theorems 2.1 and 2.3. Finally $A^{\prime}(\varrho)$ counts the number of charged constituents of $\varrho$ on all possible scales; see (3.4). In Sect. 3 we have shown that (4.1) implies

$$
z(\beta, \varrho) \leqq \exp [(c-\beta d) A(\varrho)],
$$

for some finite, positive constants $c$ and $d$, provided $z \leqq$ const $e^{\varepsilon \beta}$.

It is an immediate consequence of the definition of $A(\varrho)$ that

hence

$$
\begin{gathered}
A(\varrho) \geqq\left(n(\varrho)-\left[\ln _{2} d(\varrho)\right]\right)+2\left[\ln _{2} d(\varrho)\right] \\
\geqq(\alpha+1)\left[\ln _{2} d(\varrho)\right]>3 \ln d(\varrho),
\end{gathered}
$$

$$
z(\beta, \varrho) \leqq \exp [3(c-\beta d) \ln d(\varrho)],
$$

so that $z(\beta, \varrho)<1$, for $\beta$ large enough; $(\alpha(\varrho) \geqq 2$, for all neutral $\varrho$ !).

\subsection{Main Result}

Let $\mathscr{N}^{\prime}$ be an ensemble satisfying properties a)-c) of Theorem 2.1. Let $\tilde{\varrho}=0, \xi \varrho_{0}$, $\xi \varrho_{0}+\varrho^{\mathcal{N}^{\prime}}$, where $\xi \varrho_{0}$ satisfies Condition (2.28), (i.e. the constituents of $\xi \varrho_{0}$ are fractionally charged), and $\mathscr{N}^{\prime}, \varrho_{0}, \varrho^{\mathcal{N}^{\prime}}$ satisfy the properties listed in Theorem 2.3 .

The main result of this section is

Theorem 4.1. Let $\mathscr{N}^{\prime}$ and $\varrho$ be as above, and $\mathscr{N} \equiv \mathscr{N}^{\prime} \cup\{\tilde{\varrho}\}$. For each $\varrho \in \mathscr{N}$, let $\sigma(\varrho)$ be some real number. Then

$$
\begin{aligned}
\int & \prod_{\varrho \in \mathscr{N}}[1+K(\varrho) \cos (\phi(\varrho)+\sigma(\varrho))] d \mu_{\beta}(\phi) \\
& =\int \prod_{\varrho \in \mathscr{N}}[1+z(\beta, \varrho) \cos (\phi(\bar{\varrho})+\sigma(\varrho))] d \mu_{\beta}(\phi),
\end{aligned}
$$


where

$$
z(\beta, \varrho)=K(\varrho) \exp \left[-\beta E_{1 \mathrm{loc}}(\varrho, \mathcal{N})\right]
$$

and $E_{\text {loc }}(\varrho, \mathscr{N})$ is some function of $\varrho$ satisfying

$$
E_{\text {loc }}(\varrho, \mathscr{N}) \geqq D_{1} A^{\prime}(\varrho) .
$$

Moreover, $\varrho$ is a charge density which depends linearly on $\varrho$ and satisfies

a) $Q(\bar{\varrho})=Q(\varrho)$, and

b) $d(\bar{\varrho}) \leqq 2 d(\varrho)$ (unless $\varrho$ is charged in which case $d(\bar{\varrho})=\infty, E_{1 \mathrm{loc}}(\varrho, \mathscr{N})=\infty$ ).

Remarks. 1. The functional $E_{\text {loc }}(\varrho, \mathscr{N})$ is a "local" portion of the electrostatic selfenergy of $\varrho$ in a sea of multipoles indexed by $\mathscr{N} \backslash\{\varrho\}$. The renormalized charge density $\varrho$ and $E_{\text {loc }}(\varrho, \mathscr{N})$ will be implicitly defined in the proof of Theorem 4.1.

2. The basic idea behind Theorem 4.1 is as follows: The renormalization of the charge densities $\varrho$ and activities is done inductively in the scale of $\varrho$. Let

$$
\mathscr{N}_{\leqq k}=\left\{\varrho: \varrho \in \mathcal{N}, d(\varrho) \leqq 2^{k}\right\} .
$$

Suppose that the induction has reached scale $2^{k}$, i.e.

$$
\begin{aligned}
\int \prod_{\varrho \in \mathscr{N}} & {[1+K(\varrho) \cos (\phi(\varrho)+\sigma(\varrho))] d \mu_{\beta}(\phi) } \\
= & \int \prod_{\varrho \in \mathscr{N} \leqq k}[1+z(\beta, \varrho) \cos (\phi(\varrho)+\sigma(\varrho))] \\
& \cdot \prod_{\varrho \in \mathscr{N} \backslash \mathscr{N} \leqq k}\left[1+K(\varrho) \cos (\phi(\varrho)+\sigma(\varrho)) d \mu_{\beta}(\phi) .\right.
\end{aligned}
$$

Pick some $\varrho^{\prime}$ with $2^{k}<d\left(\varrho^{\prime}\right) \leqq 2^{k+1}$. One can, in principle, renormalize $1+K\left(\varrho^{\prime}\right) \cos \left(\phi\left(\varrho^{\prime}\right)+\sigma\left(\varrho^{\prime}\right)\right)$ by integrating over all variables $\phi(j)$, with $j$ belonging to some bounded array, $\Omega\left(\varrho^{\prime}, \mathcal{N}\right)$, of lattice sites centered around supp $\varrho^{\prime}$ and $\operatorname{diam}\left(\Omega\left(\varrho^{\prime}, \mathscr{N}\right)\right) \leqq 2 d\left(\varrho^{\prime}\right)$, in such a way that the factor

$$
\Pi^{\prime}[1+z(\beta, \varrho) \cos (\phi(\bar{\varrho})+\sigma(\varrho))],
$$

where $\Pi^{\prime}$ extends over all those $\varrho \in \mathscr{N}_{\leqq k}$ for which $\operatorname{dist}\left(\varrho, \varrho^{\prime}\right) \leqq d\left(\varrho^{\prime}\right)$, is not affected. Note that if $\varrho^{\prime \prime}$ satisfies $d\left(\varrho^{\prime \prime}\right)>2^{k}$ then

$$
\operatorname{dist}\left(\varrho^{\prime \prime}, \varrho^{\prime}\right) \geqq M 2^{\alpha k} \gg 2^{k+2} \geqq 2 d\left(\varrho^{\prime}\right), \quad \text { for } \quad M \geqq 4,
$$

so that factors $\left[1+K\left(\varrho^{\prime \prime}\right) \cos \left(\phi\left(\varrho^{\prime \prime}\right)+\sigma\left(\varrho^{\prime \prime}\right)\right)\right]$ corresponding to unrenormalized multipole densities $\varrho^{\prime \prime} \neq \varrho^{\prime}$ on scales $>2^{k}$ are not affected by the renormalization of $\varrho^{\prime}$.

Mathematically, the renormalization by integration described above corresponds to taking a conditional expectation. One might view this procedure as a "block spin transformation": the "spin" $\phi(\varrho)$ is replaced by a "spin" $\phi(\varrho))$, where $\varrho$ is a new charge density - in general not integer-valued - with supp $\varrho \subset \partial \Omega(\varrho, \mathscr{N})$.

However, it appears that explicit integration over the variables $\phi(j), j \in \Omega(\varrho, \mathscr{N})$, is an inconvenient way of carrying out the renormalization. Instead, we extract the main contribution to the integrals described above by using electrostatic inequalities, in the form of complex translations $[14,5]$. 


\subsection{Electrostatics for a Single Charge}

For the proof of Theorem 4.1 we need the following lemma.

Lemma 4.2. If $G(\phi)$ is a functional independent of $\phi\left(j_{0}\right)$ then

$$
\int e^{i q \phi\left(j_{0}\right)} G(\phi) d \mu_{\beta}(\phi)=e^{-\beta q^{2} / 8} \int e^{i q \bar{\phi}\left(j_{0}\right)} G(\phi) d \mu_{\beta}(\phi),
$$

where $\bar{\phi}\left(j_{0}\right)=1 / 4 \sum_{\left|k-j_{0}\right|=1} \phi(k)$.

Proof. The proof follows by explicit integration of the $\phi\left(j_{0}\right)$ variable:

$$
\begin{aligned}
& \int e^{i q \phi\left(j_{0}\right)} \exp \left[-\sum_{\left|k-j_{0}\right|=1}\left(\phi\left(j_{0}\right)-\phi(k)\right)^{2} / 2 \beta\right] d \phi\left(j_{0}\right) \\
& =e^{-\beta q^{2} / 8} e^{i q \bar{\phi}\left(j_{0}\right)} \int \exp \left[-\sum_{\left|k-j_{0}\right|=1}\left(\phi\left(j_{0}\right)-\phi(k)\right)^{2} / 2 \beta\right] d \phi\left(j_{0}\right) .
\end{aligned}
$$

For the purpose of applying our methods to models other than the Coulomb gas, e.g. the solid-on-solid model, or the $\mathbb{Z}_{n}$-models, we need a slight generalization of Lemma 4.2 which we now describe. We consider functions $I_{\beta}(\phi)$, (replacing the Gaussian $e^{\left.-(1 / 2 \beta) \phi^{2}\right)}$, which satisfy the following

Condition (4.10). i) $I_{\beta}(\phi)$ is an even, positive integrable function of $\phi \in \mathbb{R}$.

ii) $I_{\beta}(z)$ is analytic in $z$ in a strip $\sum_{\varepsilon}=\{\zeta:|\operatorname{Im} \zeta|<\varepsilon\}$ around the real axis, for some large constant $\varepsilon$. (When $\varepsilon=\infty, I_{\beta}$ is entire.)

iii) $I_{\beta}$ satisfies the inequality

$$
\left|I_{\beta}(\phi+i a) / I_{\beta}(\phi)\right| \leqq e^{c(\beta) a^{2}},
$$

for all a with $|a|<\varepsilon$, all $\phi \in \mathbb{R}$, where $c(\beta)$ is some $\phi$ - and a-independent constant which tends to 0 , as $\beta \rightarrow \infty ;(c(\beta)$ may depend on the choice of $\varepsilon)$.

Note that by i) and ii)

$$
\overline{I_{\beta}(\phi+i a)}=I_{\beta}(\phi-i a) .
$$

Remark. Clearly, the Gaussian

$$
I_{\beta}(\phi)=\exp \left[-(1 / 2 \beta) \phi^{2}\right]
$$

satisfies Condition (4.10), with $\varepsilon=\infty$ and $c(\beta)=1 / 2 \beta$.

We define

$$
i_{\beta}(a ; \phi) \equiv I_{\beta}(\phi+i a) I_{\beta}(\phi)^{-1} e^{c(\beta) a^{2}} .
$$

By Condition (4.10), iii), $\left\|i_{\beta}(a ; \cdot)\right\|_{\infty} \leqq 1$. Let $d \mu_{I_{\beta}}$ be any measure satisfying Dobrushin-Lanford-Ruelle (DLR) equations [16] with Hamiltonian

$$
\beta H_{\Lambda}=-\sum_{\langle i, j\rangle \subset A} \ln I_{\beta}(\phi(i)-\phi(j)),
$$


and a priori measure $\prod_{k \in A} d \phi(k)$, e.g.

$$
d \mu_{I_{\beta}}(\phi)=N_{\Lambda}^{-1} \prod_{\substack{|i-j|=1 \\ i \in \Lambda}} I_{\beta}(\phi(i)-\phi(j)) \prod_{k \in \Lambda} d \phi(k)
$$

where $N_{\Lambda}$ is the obvious normalization factor, and $\phi(j)=0$, for all $j \notin \Lambda$.

Lemma 4.3. Let $I_{\beta}$ satisfy Condition (4.10), let $d \mu_{I_{\beta}}$ and $i_{\beta}$ be as above, $j_{0} \in \Lambda$ and $G(\phi)$ independent of $\phi\left(j_{0}\right)$. Then, for all a with $|a|<\varepsilon$

$$
\int e^{i q \phi\left(j_{0}\right)} G(\phi) d \mu_{I_{\beta}}(\phi)=e^{-a q+4 c(\beta) a^{2}} \int e^{i q \phi\left(j_{0}\right)}\left[\prod_{\left|k-j_{0}\right|=1} i_{\beta}\left(a ; \phi\left(j_{0}\right)-\phi(k)\right)\right] G(\phi) d \mu_{I_{\beta}}(\phi) .
$$

Proof. Since $d \mu_{I_{\beta}}$ satisfies the DLR equations corresponding to (4.13), it suffices to consider the integral

$$
\mathscr{I} \equiv \int e^{i q \phi\left(j_{0}\right)} \prod_{\left|k-j_{0}\right|=1} I_{\beta}\left(\phi\left(j_{0}\right)-\phi(k)\right) d \phi\left(j_{0}\right) .
$$

By Condition (4.10), we may shift the contour of integration: $\phi\left(j_{0}\right) \mapsto \phi\left(j_{0}\right)+i a$, with $|a|<\varepsilon$. This yields

$$
\begin{aligned}
\mathscr{I}= & e^{-q a} \int e^{i q \phi\left(j_{0}\right)} \prod_{\left|k-j_{0}\right|=1} I_{\beta}\left(\phi\left(j_{0}\right)-\phi(k)+i a\right) d \phi\left(j_{0}\right) \\
= & e^{-q a} e^{4 c(\beta) a^{2}} \int e^{i q \phi\left(j_{0}\right)} \prod_{\left|k-j_{0}\right|=1}\left\{i_{\beta}\left(a ; \phi\left(j_{0}\right)-\phi(k)\right)\right. \\
& \left.\cdot I_{\beta}\left(\phi\left(j_{0}\right)-\phi(k)\right)\right\} d \phi\left(j_{0}\right) .
\end{aligned}
$$

[Since $\left|i_{\beta}(a ; \phi)\right| \leqq 1$, the integral on the right side of (4.15) converges absolutely, by Condition (4.10), i).]

Remarks. 1) If $c(\beta)$ is independent of $\varepsilon$, the factor

$$
e^{-q a+4 c(\beta) a^{2}}
$$

is minimized by setting $a=q / 8 c(\beta)$, provided $|q / 8 c(\beta)|<\varepsilon$, in which case the value at the minimum is given by $\exp \left[-q^{2} / 16 c(\beta)\right]$.

If $|q / 8 c(\beta)| \geqq \varepsilon$ we set $a=\varepsilon \operatorname{sign} q$. If the constant $c(\beta)$ depends on the choice of $\varepsilon$, $c(\beta)=c(\beta, \varepsilon)$ - as the case may be, e.g. in the solid-on-solid (s-o-s) and the $\mathbb{Z}_{n}$-models - the minimum is obtained by minimizing $-q a+4 c(\beta,|a|) a^{2}$. In all cases we define

$$
E(\beta, q) \equiv \max _{a}\left\{q a-4 c(\beta) a^{2}\right\},
$$

and $a(\beta, q)$ to be the maximizing choice for $a$.

2) The above proof is based on the fact that integrals of analytic functions of finitely many variables, $\phi(j)$, can be estimated by means of complex translations of the $\phi(j)$ 's, [14]. This is the principle which we shall apply to renormalize multipole densities and activities and establish Theorem 4.1. (When $I_{\beta}$ is the Gaussian, the complex translation method is essentially equivalent to using electrostatic inequalities to estimate the integrals.) 
Next, we decompose the support of a charge density $\varrho \in \mathscr{N}$ into two disjoint subsets $\Omega_{1}, \Omega_{2}$ such that no two sites in $\Omega_{1}$ are nearest neighbors. Let $\varrho_{\ell}(j)=\varrho(j)$, for $j \in \Omega_{\ell}, \varrho_{\ell}(j)=0$, otherwise, $\ell=1,2$. Clearly $\varrho_{1}+\varrho_{2}=\varrho$. We define

$$
R_{0} \varrho(j) \equiv \varrho_{2}(j)+1 / 4 \sum_{|k-j|=1} \varrho_{1}(k) .
$$

By iterated application of Lemma 4.2 we have

$$
\int \prod_{\varrho \in \mathcal{N}}(1+K(\varrho) \cos \phi(\varrho)) d \mu_{\beta}(\phi)=\int \prod_{\varrho \in \mathscr{N}}\left(1+K(\varrho) e^{-\beta E_{0}\left(\varrho_{1}\right)} \cos \phi\left(R_{0} \varrho\right)\right) d \mu_{\beta}(\phi),
$$

where $E_{0}\left(\varrho_{1}\right)=1 / 8 \sum \varrho_{1}(j)^{2}$. By an appropriate choice of $\Omega_{1}$ we have

$$
E_{0}\left(\varrho_{1}\right) \geqq \frac{1}{2} E_{0}(\varrho)
$$

In the proof of (4.18), (4.19) we have used the fact that for $M>1 \operatorname{dist}\left(\varrho, \varrho^{\prime}\right) \geqq 3$, for all $\varrho \neq \varrho^{\prime}$ in $\mathcal{N}$, so that $\operatorname{supp}\left(R_{0} \varrho\right) \cap \operatorname{supp}\left(R_{0} \varrho^{\prime}\right)=\emptyset$, for any choice of $\Omega_{\ell}(\varrho), \Omega_{\ell}\left(\varrho^{\prime}\right)$, $\ell=1,2$. Thus $\Omega_{\ell}(\varrho), \ell=1,2$, can be chosen to be independent of $\varrho^{\prime} \in \mathcal{N}, \varrho^{\prime} \neq \varrho$, and such that (4.19) holds, for all $\varrho \in \mathscr{N}$. Identity (4.18) then follows by iteration of (4.9).

We may think of $K(\varrho) e^{-\beta E_{0}\left(\varrho_{1}\right)}, R_{0} \varrho$, respectively, as the renormalization of the bare activity and charge distribution on a scale of $2^{\circ}$ (whence the symbol " $R_{0}$ "). Note that $Q\left(R_{0} \varrho\right)=Q(\varrho)$, for all $\varrho$.

Let $I_{\beta}$ be some function satisfying Condition (4.10). Let $E(\beta, q)$ and $a(\beta, q)$ be given by (4.16), and

$$
E\left(\beta, \varrho_{1}\right)=\sum E\left(\beta, \varrho_{1}(j)\right) .
$$

By a suitable choice of $\Omega_{1}$,

We define

$$
E\left(\beta, \varrho_{1}\right) \geqq \frac{1}{2} E(\beta, \varrho) .
$$

and

$$
e_{\beta}(\phi, \varrho) \equiv e^{i \phi(\varrho)} \prod_{k \in \Omega_{1}}\left\{\prod_{|j-k|=1} i_{\beta}\left(a\left(\beta, \varrho_{1}(k)\right) ; \phi(k)-\phi(j)\right)\right\}
$$

$$
c_{\beta}(\phi, \varrho)=\operatorname{Re} e_{\beta}(\phi, \varrho), \quad s_{\beta}(\phi, \varrho)=\operatorname{Im} e_{\beta}(\phi, \varrho) .
$$

By the same reasoning as above we deduce from Lemma 4.3 that

$$
\int \prod_{\varrho \in \mathcal{N}}(1+K(\varrho) \cos \phi(\varrho)) d \mu_{I_{\beta}}(\phi)=\int \prod_{\varrho \in \mathcal{N}}\left(1+K(\varrho) e^{-E\left(\beta, \varrho_{1}\right)} c_{\beta}(\phi, \varrho)\right) d \mu_{I_{\beta}}(\phi),
$$

and we have used that

$$
c_{\beta}(\phi, \varrho)=1 / 2\left(e_{\beta}(\phi, \varrho)+e_{\beta}(\phi,-\varrho)\right),
$$

an immediate consequence of (4.11). Let $\varrho$ be an arbitrary charge density of compact support. Then

$$
e_{\beta}(\phi+b, \varrho)=e^{i r Q(\varrho)} e_{\beta}(\phi, \varrho),
$$

for any function $b$ on $\mathbb{Z}^{2}$ taking the constant value $r$ on $\{j: \operatorname{dist}(j$, supp $\varrho) \leqq 1\}$. 


\subsection{The Basic Lemma Electrostatics for General Charge Densities}

Next, we generalize Lemmas 4.2 and 4.3 in a way suitable for extracting the selfenergy of charged constituents of some charge density $\varrho \in \mathscr{N}$ on scales $2^{k}$, $k=1,2,3, \ldots$ We introduce some additional notation. For any function $\varrho$ of compact support on $\mathbb{Z}^{2}$, let $D(\varrho)$ be all those sites of $\mathbb{Z}^{2}$ contained in a disc in $\mathbb{R}^{2}$ of radius $2 d(\varrho)$ centered at a point $x(\varrho)$ such that supp $\varrho \subset D(\varrho)$, and

$$
\operatorname{dist}(\operatorname{supp} \varrho, \partial D(\varrho))=d(\varrho) \text {; }
$$

[here $\partial D(\varrho)$ is the outer boundary of $D(\varrho)$ ]. Now let us fix a particular charge density $\varrho^{*} \in \mathscr{N}$, with $Q\left(\varrho^{*}\right)=0$. We define

$$
\mathscr{N}^{*}=\left\{\varrho: \varrho \in \mathscr{N}, Q(\varrho)=0, d(\varrho) \leqq 2 d\left(\varrho^{*}\right), \varrho \neq \varrho^{*}\right\} .
$$

[If $\mathcal{N}$ contains a charged element, $\varrho_{c}, \varrho_{c} \notin \mathscr{N}^{*}$, for any choice of $\varrho^{*} \in \mathscr{N}$. The factor $K\left(\varrho_{c}\right) \cos \left(\phi\left(\varrho_{c}\right)+\sigma\left(\varrho_{c}\right)\right)$ can be renormalized to 0 , after all factors indexed by $\varrho \in \mathscr{N} \backslash\left\{\varrho_{c}\right\}$ have been renormalized.] We consider a mapping, $G$, from $\mathscr{N}^{*}$ into functions of the variables $\{\phi(j)\}$,

with the properties

$$
\varrho \in \mathscr{N}^{*} \mapsto G(\phi, \varrho),
$$

i) $G(\phi, \varrho)$ only depends on the variables $\{\phi(j): j \in D(\varrho)\}$,

ii) $G(\phi+b, \varrho)=G(\phi, \varrho)$,

for any function $b$ on $\mathbb{Z}^{2}$ taking an arbitrary, constant value, $r$, on $D(\varrho)$.

We should think of $G(\phi, \varrho)$ as the renormalized version, or "block spin transform" of $1+K(\varrho) \cos (\phi(\varrho)+\sigma(\varrho)), \varrho \in \mathscr{N}^{*}$. In the Gaussian case, i.e. $I_{\beta}(\phi)$ $=\exp \left[-(1 / 2 \beta) \phi^{2}\right], d \mu_{I_{\beta}}=d \mu_{\beta}, G$ has the form

$$
G(\phi, \varrho)=1+z(\beta, \varrho) \cos (\phi(\varrho)+\sigma(\varrho)), \text { or }=1+K(\varrho) \cos (\phi(\varrho)+\sigma(\varrho)),
$$

where $\varrho$ is some linear function of $\varrho$, and

$$
z(\beta, \varrho)=K(\varrho) e^{-\beta E_{10 \mathrm{c}(\varrho, \mathcal{N})}} .
$$

[The motivation for the generality of our presentation lies in the circumstance that in many interesting models, e.g. in the s-o-s and the $\mathbb{Z}_{n}$-models, $I_{\beta}$ is not Gaussian and, as a consequence, the renormalized version of $1+K(\varrho) \cos (\phi(\varrho)+\sigma(\varrho))$ is not of the form (4.29).] We now define

$$
F_{\mathscr{N}^{*}}(\phi)=\prod_{\varrho \in \mathcal{I}^{*}} G(\phi, \varrho) \prod_{\varrho^{*} \neq \varrho \in \mathscr{N} \backslash \mathcal{N}^{*}}[1+K(\varrho) \cos (\phi(\varrho)+\sigma(\varrho))] .
$$

Let $\Omega_{1}$, be the set of all sites contained in a disc of radius $R_{1}$ in $\mathbb{R}^{2}$ centered at some point $x_{1}$, with

$$
\Omega_{1} \subset D\left(\varrho^{*}\right), \text { and } \operatorname{supp} \varrho^{*} \cap \Omega_{1} \neq \emptyset .
$$

Let $\Omega_{2}$ be some other subset of $D\left(\varrho^{*}\right)$, disjoint from $\Omega_{1}$, such that

$$
\operatorname{dist}\left(x_{1}, \partial \Omega_{2}\right) \geqq R R_{1}, \quad R \geqq \sqrt{2} .
$$

Let $C_{i}\left(\phi, \varrho^{*}\right)$ be some bounded function only depending on $\left\{\phi(j): j \in \Omega_{i}\right\}, i=1,2$. We further assume that $C_{1}\left(\phi, \varrho^{*}\right)$ has the properties

$$
C_{1}\left(\phi+b, \varrho^{*}\right)=e^{i q r} C_{1}\left(\phi, \varrho^{*}\right),
$$


for any function $b$ taking the constant value $r$ on $\Omega_{1}$, where $q=\sum_{j \in \Omega_{1}} \varrho^{*}(j)$, and

$$
\overline{C_{1}\left(\phi, \varrho^{*}\right)}=C_{1}\left(\phi,-\varrho^{*}\right) .
$$

Example. In the Gaussian case,

$$
C_{1}\left(\phi, \varrho^{*}\right)=e^{i \phi(f)} e^{i \sigma\left(\varrho^{*}\right)},
$$
where $f$ and $\sigma\left(\varrho^{*}\right)$ are linear functions of $\varrho^{*}, \operatorname{supp} f \subseteq \Omega_{1}$, and $\sum_{j \in \Omega_{1}} f(j)=\sum_{j \in \Omega_{1}} \varrho^{*}(j)$
$=q$.

Lemma 4.4. Let $\mathcal{N}$ be as in Theorem 4.1, and let $\alpha$ and $M$ be the constants introduced in Sect. 2, Theorem 2.1,(2.4) and (2.5). Let $\mathscr{N}^{*}, \varrho^{*}, F_{\mathcal{N}^{*}}, C_{1}$, and $C_{2}$ be as specified above. Let $\varepsilon$ be the width of the strip on which $I_{\beta}$ satisfies Condition (4.10). Then, for $\alpha>3 / 2$ and $M$ sufficiently large.

$$
\int C_{1}\left(\phi, \varrho^{*}\right) C_{2}\left(\phi, \varrho^{*}\right) F_{\mathcal{N}^{*}} d \mu_{I_{\beta}}(\phi)=e\left(C_{1}, \beta\right) \int\left(R C_{1}\right)\left(\phi, \varrho^{*}\right) C_{2}\left(\phi, \varrho^{*}\right) F_{\mathcal{N}^{*}}(\phi) d \mu_{I_{\beta}}(\phi),
$$

where

a) $e$ is a numerical factor satisfying

$$
0 \leqq e\left(C_{1}, \beta\right) \leqq \min _{\gamma: \gamma \cdot k\left(R_{1}\right) \leqq \varepsilon}\left\{\exp \left[-\ln R\left(\gamma q-c(\beta) \gamma^{2} d(M)\right)\right]\right\}
$$

for some constants $d(M)$ and $k\left(R_{1}\right)$, with $d(M) \rightarrow 2 \pi+1$, as $M \rightarrow \infty, k\left(R_{1}\right)=\min (\ln R$, $K \cdot R_{1}^{(1 / \alpha)-1}$ ), where $K$ only depends on $M$ and $R$, and

b) $R C_{1}$ is a function, defined in (4.40) below, only depending on the variables $\left\{\phi(j): j \in R \Omega_{1}\right\}$, with $R \Omega_{1} \equiv\left\{j: \operatorname{dist}\left(j, x_{1}\right) \leqq R R_{1}+1\right\}$, with the properties

$$
\begin{gathered}
\left\|\left(R C_{1}\right)\left(\cdot, \varrho^{*}\right)\right\|_{\infty} \leqq\left\|C_{1}\left(\cdot, \varrho^{*}\right)\right\|_{\infty}, \\
\left(R C_{1}\right)\left(\phi+b, \varrho^{*}\right)=e^{i q r}\left(R C_{1}\right)\left(\phi, \varrho^{*}\right),
\end{gathered}
$$

for any function $b$ taking the constant value $r$ on $R \Omega_{1}$, and

$$
\overline{\left(R C_{1}\right)\left(\phi, Q^{*}\right)}=\left(R C_{1}\right)\left(\phi,-Q^{*}\right) \text {. }
$$

Remark. $R C_{1}$ should be thought of as the renormalization of $C_{1}$. The lemma will enable us to renormalize $1+K\left(\varrho^{*}\right) \cos \left(\phi\left(\varrho^{*}\right)+\sigma\left(\varrho^{*}\right)\right)$ inductively, the induction extending over all scales of $2^{k}, k=2,3, \ldots$.

More precisely, in the proof of Theorem 4.1, Lemma 4.4 is needed to carry out the induction step, $2^{k} \rightarrow 2^{k+1}$. The purpose of the renormalization transformations is to replace $1+K\left(\varrho^{*}\right) \cos \left(\phi\left(\varrho^{*}\right)+\sigma\left(\varrho^{*}\right)\right)$ by a positive function of $\phi$, close to the identity.

Proof of Lemma 4.4. For notational simplicity we suppose that $\Omega_{1}$ is centered at the origin, i.e., $x_{1}=0$. Let $b(j)$ be a real-valued function on $\mathbb{R}^{2}$ defined by

$$
b(j)= \begin{cases}\ln R, & |j| \leqq R_{1} \\ \ln \left[R R_{1}|j|^{-1}\right], & R_{1} \leqq|j| \leqq R R_{1} \\ 0, & |j| \leqq R R_{1} .\end{cases}
$$

We make the following change of variables:

$$
\phi(j) \mapsto \phi(j)+i a(j),
$$

where $a$ is a real-valued function defined in terms of $b$ as follows: 
Let $C_{i}$ be the connected components of $\bigcup_{\varrho \in \mathcal{N}^{*}} D(\varrho)$. We express each $C_{i}$ as a union of $D(\varrho), \varrho \in S_{i} \subset \mathscr{N}^{*}$. For each $S_{i}$ we shall show that there is a unique $\varrho_{i} \in S_{i}$ of maximal diameter. We now define

$$
a(j)= \begin{cases}\gamma b(j), & \text { if } j \notin \bigcup_{\varrho \in \mathcal{N}^{*}} D(\varrho) \\ \gamma b\left(x_{i}\right), & \text { if } j \in C_{i},\end{cases}
$$

where $x_{i}$ is a point of $C_{i}$ chosen so that if $C_{i}$ meets $|k| \leqq R_{1}, a(j) \equiv \gamma \ln R$ and if $C_{i}$ meets $|k| \geqq R R_{1} a(j) \equiv 0$, for all $j \in C_{i}$. Otherwise we set $x_{i}=x\left(\varrho_{i}\right)$, the center of $D\left(\varrho_{i}\right)$. In Appendix E we establish two properties of $C_{i}$

a) $\operatorname{diam} C_{i} \leqq \frac{5}{2} d\left(\varrho_{i}\right)$,

b) $\left|\partial C_{i}\right| \leqq 10 d\left(\varrho_{i}\right)$.

The uniqueness of $\varrho_{i}$ follows immediately from a) and (2.4). Note that since $R_{1}$ $\leqq d\left(\varrho^{*}\right),(2.4)$ together with a) imply that $\operatorname{diam} C_{i} \ll R_{1}$ hence for $R \geqq \sqrt{2}, C_{i}$ cannot meet both $|k| \leqq R_{1}$ and $|k| \geqq R R_{1}$ hence $a(j)$ is well defined.

The constant $\gamma$ is chosen such that

$$
\max _{\substack{|j-k|=1 \\ R_{1} \leqq|j| \leqq R R_{1}}}|a(j)-a(k)| \leqq \varepsilon,
$$

where $\varepsilon$ is the width of the strip of analyticity of the function $I_{\beta}$. Thus, by Condition (4.10), the change of variables (4.36) is permitted. It follows from Theorem $2.1, b),(2.4)$ and the properties a) and b) that

$$
\gamma^{-1} \max _{\substack{|j-k|=1 \\ R_{1} \leqq|j| \leqq R R_{1}}}|a(j)-a(k)| \leqq \min \left(\ln R, K R_{1}^{(1 / \alpha)-1}\right),
$$

with $K=\max \left(1, \operatorname{const}(R / M)^{1 / \alpha}\right)$, so that $\gamma \leqq \varepsilon k\left(R_{1}\right)^{-1}$ suffices.

Note that $F_{\mathcal{N}^{*}}$ is unaffected by the change of variables. This is seen as follows: By property (4.28) of $G(\phi, \varrho), \varrho \in \mathscr{N}^{*}$, and definition (4.37)

$$
G(\phi+i a, \varrho)=G(\phi, \varrho) .
$$

If $\varrho \notin \mathscr{N}^{*}, d(\varrho)>2 d\left(\varrho^{*}\right)$. Thus

$$
\operatorname{dist}\left(\varrho, \varrho^{*}\right) \geqq M d\left(\varrho^{*}\right)^{\alpha}>R R_{1}
$$

provided $M$ is large enough and $\alpha>1$. [Note that $R R_{1} \leqq \operatorname{diam}\left(D\left(\varrho^{*}\right)\right)-d\left(\varrho^{*}\right)$ $\leqq 3 d\left(\varrho^{*}\right)$, so $M>3$ suffices.] Hence $a(j)=0$, for all $j \in \operatorname{supp} \varrho$, all $\varrho \in \mathscr{N} \backslash \mathscr{N}^{*}, \varrho \neq \varrho^{*}$. Thus, only $C_{1}\left(\phi, \varrho^{*}\right)$ and $d \mu_{I_{\beta}}(\phi)$ are affected by the change of variables (4.36);

$$
d \mu_{I_{\beta}}(\phi+i a)=\left\{\prod_{\substack{k-j|=1 \\ j| \leqq R R_{1}}} i_{\beta}(a(j)-a(k) ; \phi(j)-\phi(k)) \exp \left[c(\beta)(a(j)-a(k))^{2}\right]\right\} d \mu_{I_{\beta}}(\phi),
$$

as follows from (4.12) and (4.13). By (4.32) and the definition of $a,(4.35),(4.37)$,

$$
C_{1}\left(\phi+i a, \varrho^{*}\right)=e^{-\gamma(\ln R) q} C_{1}\left(\phi, \varrho^{*}\right) .
$$

We set

$$
\left(R C_{1}\right)\left(\phi, \varrho^{*}\right)=\left\{\prod_{\substack{k-j|=1\\| j \mid \leqq R R_{1}}} i_{\beta}(a(j)-a(k) ; \phi(j)-\phi(k))\right) C_{1}\left(\phi, \varrho^{*}\right)
$$


and

$$
e\left(C_{1}, \beta\right)=e^{-\gamma(\ln R) q} \exp \left[c(\beta)\|\nabla a\|_{2}^{2}\right] .
$$

Since, by (4.12), $\left\|i_{\beta}(a ; \cdot)\right\|_{\infty} \leqq 1$,

$$
\left\|\left(R C_{1}\right)\left(\cdot, \varrho^{*}\right)\right\|_{\infty} \leqq\left\|C_{1}\left(\cdot, \varrho^{*}\right)\right\|_{\infty} .
$$

Next, if $b$ is a function which is constant on $R \Omega_{1}$ then

$$
\prod_{\substack{k-j|=1 \\ j| \leqq R R_{1}}} i_{\beta}(a(j)-a(k) ; \phi(j)-\phi(k)+b(j)-b(k))=\prod_{\substack{k-j|=1\\| j \mid \leqq R R_{1}}} i_{\beta}(a(j)-a(k) ; \phi(j)-\phi(k)),
$$

so that

$$
\left(R C_{1}\right)\left(\phi+b, \varrho^{*}\right)=e^{i q r}\left(R C_{1}\right)\left(\phi, \varrho^{*}\right),
$$

where $r$ is the value of $b$ on $R \Omega_{1}$. Finally,

$$
\begin{aligned}
\overline{\left(R C_{1}\right)\left(\phi, \varrho^{*}\right)} & =\prod_{|k-j|=1} \overline{i_{\beta}(a(j)-a(k) ; \phi(j)-\phi(k))} \overline{C_{1}\left(\phi, \varrho^{*}\right)} \\
& =\prod_{|k-j|=1} i_{\beta}(-a(j)+a(k), \phi(j)-\phi(k)) C_{1}\left(\phi,-\varrho^{*}\right) \\
& =\left(R C_{1}\right)\left(\phi,-\varrho^{*}\right),
\end{aligned}
$$

if $-a$ is chosen as a translation function when $\varrho^{*}$ is replaced by $-\varrho^{*}$ which is what will be required henceforth.

It remains to prove estimate (4.34) on $e\left(C_{1}, \beta\right)$ which is really the essential part of Lemma 4.4

By definition (4.37)

$$
\sum_{j}(\nabla a)^{2}(j)=\gamma^{2} \sum_{\substack{j \notin \cup D(\varrho) \\ \varrho \in \mathcal{N}^{*}}}(\nabla b)^{2}(j)+\sum_{i} B_{i},
$$

where

$$
B_{i}=\gamma^{2} \sum_{j \in \partial C_{i}}\left(b\left(x_{i}\right)-b(j)\right)^{2} .
$$

The first term on the right side of (4.42) is bounded above by

$$
\gamma^{2} \sum_{j}(\nabla b)^{2}(j) \leqq \gamma^{2}(2 \pi+1) \ln R,
$$

as can be seen by comparing the finite difference gradient with the continuum gradient, i.e. by estimating $\sum_{j}(\nabla b)^{2}(j)$ in terms of $\int_{R_{1} \leqq|x| \leqq R R_{1}}(\nabla b)^{2}(x) d^{2} x$.

By (4.43) and b), $B_{i}$ is bounded above by

$$
\begin{aligned}
B_{i} & \leqq \gamma^{2} 10 d\left(\varrho_{i}\right) \max _{j \in \partial C_{i}}\left[b\left(x_{i}\right)-b(j)\right]^{2} \\
& \leqq \gamma^{2} 20 d\left(\varrho_{i}\right)\left[\ln \left|x_{i}\right|-\ln \left(\left|x_{i}\right|-3 d\left(\varrho_{i}\right)\right)\right]^{2} \\
& \leqq 180 \gamma^{2} d\left(\varrho_{i}\right)^{3}\left[\left|x_{i}\right|-3 d\left(\varrho_{i}\right)\right]^{-2} .
\end{aligned}
$$

Note that $B_{i}=0$, unless

$$
R_{1}-3 d\left(\varrho_{i}\right) \leqq\left|x_{i}\right| \leqq R R_{1}+3 d\left(\varrho_{i}\right)
$$


otherwise $C_{i}$ lies entirely outside the annulus $R_{1} \leqq|j| \leqq R R_{1}$ hence $b\left(x_{i}\right)=b(j)$, $j \in \partial C_{i}$.

In order to bound the sum of the $B_{i}$ we first show that $d\left(\varrho_{i}\right)$ is small compared to $R_{1}$. By (2.4)

$$
M d^{\alpha}<\operatorname{dist}\left(\varrho_{i}, \varrho^{*}\right) \text {, with } d=\min \left[d\left(\varrho_{i}\right), d\left(\varrho^{*}\right)\right] .
$$

By a simple, geometric argument and (4.46)

$$
\operatorname{dist}\left(\varrho_{i}, \varrho^{*}\right) \leqq R_{1}+\left|x_{i}\right|+d\left(\varrho_{i}\right) \leqq 2\left|x_{i}\right|+4 d\left(\varrho_{i}\right) .
$$

Using the upper bound in (4.46) we thus get

$$
\begin{aligned}
M d^{\alpha} & <2\left|x_{i}\right|+4 d\left(\varrho_{i}\right) \leqq 2 R R_{1}+8 d\left(\varrho_{i}\right) \\
& \leqq 2 R R_{1}+16 d\left(\varrho^{*}\right) \leqq 24 d\left(\varrho^{*}\right),
\end{aligned}
$$

since $d(\varrho) \leqq 2 d\left(\varrho^{*}\right)$, for all $\varrho \in \mathscr{N}^{*}$, and

$$
R R_{1}<\operatorname{diam} D\left(\varrho^{*}\right) \leqq 4 d\left(\varrho^{*}\right) .
$$

Thus, for $\alpha \geqq 1$ and $M$ large enough, $d=d\left(\varrho_{i}\right)$. We then get from (4.47)

$$
\frac{M-4}{2} d\left(\varrho_{i}\right)^{\alpha}<\left|x_{i}\right|
$$

and

$$
\frac{M-8}{2} d\left(\varrho_{i}\right)^{\alpha}<R R_{1} .
$$

From (4.48) and (4.46) we conclude that for $M$ large enough, $\alpha>1$, and for all $\varrho_{i}$ for which $B_{i} \neq 0$,

$$
(1-\delta) R_{1} \leqq\left|x_{i}\right| \leqq R R_{1}(1+\delta),
$$

for some $\delta=\delta(M, \alpha)$ which tends to 0 , as $M \rightarrow \infty$. Moreover, (4.48) yields

$$
\left|x_{i}\right|-2 d\left(\varrho_{i}\right) \geqq \frac{1}{\sqrt{2}}\left|x_{i}\right|,
$$

for $M$ large enough.

Next, let $\mathscr{N}_{k}^{*}$ be the class of all those $\varrho_{i} \in \mathscr{N}^{*}$ satisfying (4.50) and whose diameters lie in the interval

$$
2^{k} \leqq d\left(\varrho_{i}\right)<2^{k+1} .
$$

Note that, by inequality (4.49),

$$
2^{k}<\left(\frac{2 R R_{1}}{M-8}\right)^{1 / \alpha}
$$

We now insert inequality (4.51) into the upper bound (4.45) for $B_{i}$. This yields

$$
\begin{aligned}
\sum_{\varrho_{1} \in \mathcal{N}_{k}^{*}} B_{i} & \leqq \operatorname{const} \gamma^{2} \sum_{\varrho_{i} \in \mathcal{N}_{k}^{*}} d\left(\varrho_{i}\right)^{3}\left|x_{i}\right|^{-2} \\
& \leqq \operatorname{const} \gamma^{2} 2^{3(k+1)} \sum_{\varrho_{i} \in \mathcal{N}_{k}^{*}}\left|x_{i}\right|^{-2} .
\end{aligned}
$$


The first inequality in (4.47) gives

$$
\left|x_{i}\right| \geqq \frac{M-4}{2} d\left(\varrho_{i}\right)^{\alpha} \geqq c_{k}, \quad \text { with } \quad c_{k} \equiv \frac{M-4}{2} 2^{\alpha k}, \text { for all } \varrho_{i} \in \mathcal{N}_{k}^{*} .
$$

Applying part b) of Theorem 2.1, (2.4), once more we see that

$$
\begin{aligned}
\left|x\left(\varrho_{1}\right)-x\left(\varrho_{2}\right)\right| & \geqq \operatorname{dist}\left(\varrho_{1}, \varrho_{2}\right)-d\left(\varrho_{1}\right)-d\left(\varrho_{2}\right) \\
& \geqq M 2^{\alpha k}-2^{k+2} \\
& >(M-4) 2^{\alpha k}, \text { for } \quad \alpha>1,
\end{aligned}
$$

for arbitrary $\varrho_{1}$ and $\varrho_{2}$ in $\mathscr{N}_{k}^{*}$ with $\varrho_{1} \neq \varrho_{2}$. This inequality and a simple, geometric consideration show that the number of elements $\varrho_{i} \in \mathscr{N}_{k}^{*}$ for which

$$
r c_{k} \leqq\left|x_{i}\right| \leqq(r+1) c_{k}
$$

is bounded by $K_{1} r$, for some constant $K_{1}$ independent of $\varrho_{i}$, $\alpha$, and $M$, provided $M$ is sufficiently large. By (4.54) and (4.50) the range of values of $r$ is given by

$$
m_{1} \leqq r \leqq m_{2},
$$

where

$$
\begin{aligned}
& m_{1}=c_{k}^{-1} \max \left((1-\delta) R_{1}, c_{k}\right) \\
& m_{2}=c_{k}^{-1} \max \left((1+\delta) R R_{1}, c_{k}\right) .
\end{aligned}
$$

Recalling the definition (4.54) of $c_{k}$, we therefore obtain

$$
\begin{aligned}
\sum_{\varrho_{i} \in \mathscr{N}_{k}^{*}}\left|x_{i}\right|^{-2} & \leqq K_{1} c_{k}^{-2} \sum_{r=m_{1}}^{m_{2}} r^{-1} \\
& \leqq\left(K_{2} / M^{2}\right) 2^{-2 \alpha k} \ln R .
\end{aligned}
$$

Combining this inequality with (4.53) we get

$$
\sum_{\varrho_{i} \in \mathscr{N}_{k}^{*}} B_{i} \leqq\left(K_{3} / M^{2}\right) \gamma^{2} \ln R 2^{(3-2 \alpha) k} .
$$

Hence, for $\alpha>3 / 2$ and $M$ sufficiently large

$$
\begin{aligned}
\sum_{\varrho_{i} \in \mathcal{N}^{*}} B_{i} & \leqq\left(K_{3} / M^{2}\right) \gamma^{2} \ln R \sum_{k=0}^{\infty} 2^{(3-2 \alpha) k} \\
& \leqq K(M) \gamma^{2} \ln R
\end{aligned}
$$

for some finite constant $K(M)$ which tends to 0 , as $M \rightarrow \infty$. If we now combine expression (4.41) for $e\left(C_{1}, \beta\right)$ with estimates (4.42), (4.44), and (4.56) we obtain inequality (4.34) of Lemma 4.4, under the condition that $\alpha>3 / 2$. This completes the proof.

\subsection{Proof of Theorem 4.1}

The proof is based on an induction in the size of the diameters of the multipole densities $\varrho \in \mathscr{N}$. We define the diameter of the charged multipole to be infinite. [This is permitted by Theorem $2.1, \mathrm{c})$.] 
Let

$$
\mathcal{N}_{\leqq p}=\left\{\varrho \in \mathscr{N}: d(\varrho) \leqq 2^{p}\right\} .
$$

The induction hypothesis is

$$
\begin{aligned}
& \int \prod_{\varrho \in \mathcal{N}}[1+K(\varrho) \cos (\phi(\varrho)+\sigma(\varrho))] d \mu_{\beta}(\phi) \\
& =\int \prod_{\varrho \in \mathscr{N} \leqq p}[1+z(\beta, \varrho) \cos (\phi(\bar{\varrho})+\sigma(\varrho))] \\
& \cdot \prod_{\varrho \in \mathcal{N} \backslash \mathscr{N}_{\leqq p}}[1+K(\varrho) \cos (\phi(\varrho)+\sigma(\varrho))] d \mu_{\beta}(\phi),
\end{aligned}
$$

where $z(\beta, \varrho)$ satisfies (4.6) and $\varrho$ has properties a) and b) stated in Theorem 4.1.

The step $p=1 \rightarrow p=2$ follows from Lemma 4.2. Now let $\varrho^{*} \in \mathcal{N}$ be such that $2^{p}$ $<d\left(\varrho^{*}\right) \leqq 2^{p+1}$. (If there is no such element we proceed to the next scale.) In order to establish Theorem 4.1, we need only show

$$
K(\varrho) \int e^{i \phi\left(\varrho^{*}\right)} F_{\mathscr{N}^{*}}(\phi) d \mu_{\beta}(\phi)=z(\varrho, \beta) \int e^{i \phi\left(\varrho^{*}\right)} F_{\mathcal{N}^{*}}(\phi) d \mu_{\beta}(\phi),
$$

where $F_{\mathcal{N}^{*}}$ is given by $(4.30)$, with $I_{\beta}(\phi)=\exp \left[-(1 / 2 \beta) \phi^{2}\right]$, and $E_{\mathrm{loc}}\left(\varrho^{*}, \mathcal{N}\right)$ $\geqq D_{1} A^{\prime}(\varrho)$.

The proof of (4.57) again proceeds by induction. First, we apply (4.18) and (4.19). It then suffices to show

$$
E_{\mathrm{loc}}\left(\varrho^{*}, \mathscr{N}\right)-\frac{1}{2} E_{0}\left(\varrho^{*}\right) \geqq D \sum_{k=1}^{\infty}\left|\mathscr{S}_{k}^{\prime}\left(\varrho^{*}\right)\right| .
$$

Let $k_{0}$ be the smallest integer such that $\mathscr{S}_{k_{0}}^{\prime}\left(\varrho^{*}\right)$ is non-empty. Let $s \in \mathscr{S}_{k_{0}}^{\prime}\left(\varrho^{*}\right)$. Recall, $\varrho^{*} / s$ is charged and isolated in the sense of (3.3). We suppose, to simplify notation, that the square is centered at the origin. Let $\bar{s}=2 s \in \mathscr{S}_{k_{0}+1}\left(\varrho^{*}\right)$. [Often $\bar{s}$ is also in $\mathscr{S}_{k_{0}+1}^{\prime}\left(\varrho^{*}\right)$, but this need not always be so.] We define

and

$$
f=R_{0}\left(\varrho^{*} / s\right)=R_{0}\left(\varrho^{*} / \bar{s}\right)
$$

In (4.32), (4.33), set

$$
g=R_{0}\left(\varrho^{*}\right)-f
$$

$$
C_{1}\left(\phi, \varrho^{*}\right)=e^{i \phi(f)}, \quad \text { and } \quad C_{2}\left(\phi, \varrho^{*}\right)=e^{i \phi(g)} .
$$

Circumscribe $s$ by a disc $\Omega_{1}$ of radius $R_{1}=\sqrt{2}\left(2^{k_{0}}-1\right)$. In (4.31), (4.31') and Lemma 4.4 , set $R=\sqrt{2}$. When $I_{\beta}(\phi)=\exp \left[-(1 / 2 \beta) \phi^{2}\right], \varepsilon=\infty$ and $c((\beta)=1 / 2 \beta$, in Condition (4.10). Furthermore $d(M)=2(\pi+1)$, for $M$ large enough, in inequality (4.34), Lemma 4.4. Hence

$$
e\left(C_{1}, \beta\right)=e^{-(\ln 2)(\beta / 4(\pi+1)) q^{2}},
$$

with $|q| \geqq \min \left(\xi^{\prime}, 1-\xi^{\prime}\right)$, when $\varrho^{*}=\bar{\varrho}$; see Condition $(2.28)$, and $|q| \geqq 1$, for all $\varrho^{*} \neq \tilde{\varrho}$.

When $C_{1}\left(\phi, \varrho^{*}\right)=e^{i \phi(f)}$ is renormalized, it is replaced by $\left(R C_{1}\right)\left(\phi, \varrho^{*}\right)$, defined in (4.40), which in the Gaussian case is given by

$$
\left(R C_{1}\right)\left(\phi, \varrho^{*}\right)=e^{i \phi(\bar{f})},
$$

for some function $\bar{f}$ depending linearly on $f$, with $\operatorname{supp} \bar{f} \subseteq \bar{s} \in \mathscr{S}_{k_{0}+1}\left(\varrho^{*}\right)$. 
This procedure clearly assigns every $s \in \mathscr{S}_{k_{0}}^{\prime}\left(\varrho^{*}\right)$ a factor $e^{-\beta D q^{2}}$, with $D=(\ln 2) / 4(\pi+1)$, and

$$
|q| \geqq \begin{cases}\min \left(\xi^{\prime}, 1-\xi^{\prime}\right), & \varrho^{*}=\tilde{\varrho} \\ 1, & \varrho^{*} \neq \tilde{\varrho}\end{cases}
$$

and if $\bar{s} \in \mathscr{S}_{k_{0}+1}^{\prime}\left(\varrho^{*}\right)$, the procedure can be iterated, since supp $\bar{f} \cong \bar{S}$.

Thus (4.57) follows by induction, and the proof of the theorem is complete.

The following Fig. 1 shows the geometric situation.

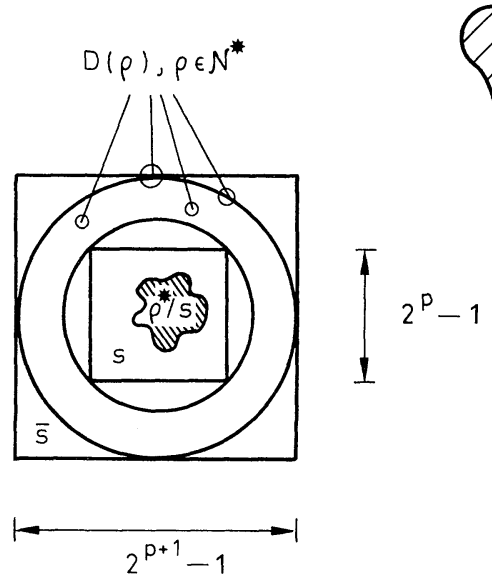

Fig. 1
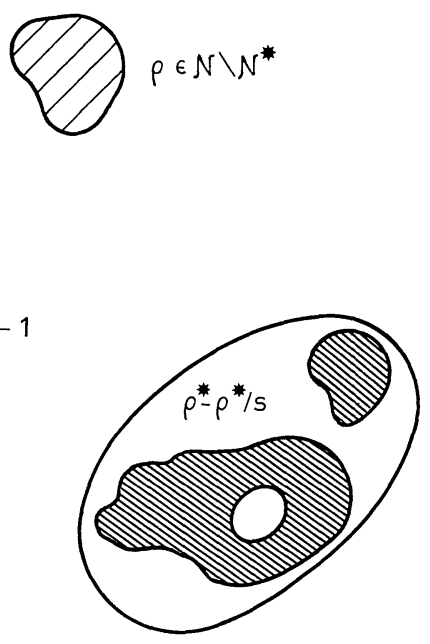

\section{Kosterlitz-Thouless Transition in the Two-Dimensional Coulomb Gas and Villain Model}

In this section we first complete our proofs of Theorems A and B (Sect. 1.3) for the hard core Coulomb gas. We then show how to extend our methods to general twodimensional Coulomb gases with a priori charge distribution $d \lambda(q)$ satisfying Condition $\lambda$, Sect. 1.2. As corollaries we obtain proofs of Theorems B-D (Sect. 1.4) for the Villain model and the dual discrete Gaussian model. The general ideas underlying our proofs are the ones described in Sect. 1.5, all principal tools have been constructed in Sects. 2-4.

\subsection{The Hard Core Coulomb Gas}

As in Sects. 2-4, we start by considering the simplest system exhibiting a Kosterlitz-Thouless transition, the two-dimensional hard core Coulomb (lattice) gas. For this gas

$$
\lambda(0)=1, \quad \lambda( \pm 1)=z / 2, \quad \lambda(q)=0, \quad \text { otherwise },
$$

and

$$
\hat{\lambda}(\phi)=(1+z \cos \phi) .
$$


Let

$$
\langle\cdot\rangle_{\Lambda}(\beta, z) \equiv\langle\cdot\rangle_{\Lambda}^{\phi}(\beta, \lambda)
$$

be the Gibbs state of the hard core gas in a finite volume $\Lambda$, with free (or periodic) b.c. at $\partial \Lambda$, in the sine-Gordon representation.

The first main result is

Theorem 5.1. Let $f$ be a real valued function such that

$$
\sum f(j)=0
$$

For each $\delta>0$ there is a $\beta_{0}$ independent of $\Lambda$ such that for all $\beta>\beta_{0}$ and $0 \leqq z$ $\leqq e^{(\beta / 17)+\alpha}$

$$
\left\langle e^{\varepsilon \phi(f)}\right\rangle_{\Lambda}(\beta, z) \geqq \exp \left[-\frac{\varepsilon^{2} \beta}{2}(1-\delta)\left\langle f, \Delta^{-1} f\right\rangle\right] .
$$

Remark. To prove Theorem A we first note that the right and left side of (5.4) are analytic functions in $\varepsilon$ and using the $\phi \rightarrow-\phi$ symmetry of $\langle\cdot\rangle(\beta, z)$ we obtain

$$
1+\frac{\varepsilon^{2}}{2}\left\langle\phi(f)^{2}\right\rangle(\beta, z)+O\left(\varepsilon^{4}\right) \geqq 1-\frac{\varepsilon^{2}}{2}(1-\delta) \beta\left\langle f, \Delta^{-1} f\right\rangle+O\left(\varepsilon^{4}\right) .
$$

Subtracting 1 from both sides, dividing by $\varepsilon^{2}$ and letting $\varepsilon \rightarrow 0$ one obtains Theorem A.

In order to prove (5.4), we consider

$$
\begin{aligned}
Z_{\Lambda}(\varepsilon f) & \equiv Z_{\Lambda}\left\langle e^{\varepsilon \phi(f)}\right\rangle_{\Lambda}(\beta, z) \\
& =\int e^{\varepsilon \phi(f)} \prod_{j \in \Lambda}(1+z \cos \phi(j)) d \mu_{\beta}(\phi) .
\end{aligned}
$$

We define

$$
\sigma_{\beta}(j)=\varepsilon \beta \sum_{\ell} C(j-\ell) f(\ell)
$$

where $C$ is the Green's function of $\Delta ; \sigma_{\beta}$ is well defined since $\hat{f}(0)=0$ and supp $f \cong \Lambda$. In (5.6) we now change variables,

$$
\phi(j) \rightarrow \phi(j)+\sigma_{\beta}(j), \text { for all } j .
$$

Under this change of variables,

$$
\begin{gathered}
e^{\varepsilon \phi(f)} \rightarrow e^{\varepsilon \phi(f)} e^{\varepsilon^{2} \beta\left\langle f,(-\Delta)^{-1} f\right\rangle}, \\
d \mu_{\beta}(\phi) \rightarrow e^{(1 / 2 \beta)\left\langle\sigma_{\beta}, \Delta \sigma_{\beta}\right\rangle} e^{(1 / \beta)\left\langle\phi, \Delta \sigma_{\beta}\right\rangle} d \mu_{\beta}(\phi), \\
\cos \phi(j) \rightarrow \cos \left(\phi(j)+\sigma_{\beta}(j)\right),
\end{gathered}
$$

where by (5.7)

$$
(1 / \beta)\left\langle\phi, \Delta \sigma_{\beta}\right\rangle=-\varepsilon \phi(f),
$$

and

$$
(1 / 2 \beta)\left\langle\sigma_{\beta}, \Delta \sigma_{\beta}\right\rangle=-\varepsilon^{2} \beta / 2\left\langle f,(-\Delta)^{-1} f\right\rangle .
$$


Thus

$$
\begin{aligned}
Z_{\Lambda}(\varepsilon f)= & e^{\left(\varepsilon^{2} \beta / 2\right)\left\langle f,(-\Delta)^{-1} f\right\rangle} \\
& \cdot \int \prod_{j \in \Lambda}\left[1+z \cos \left(\phi(j)+\sigma_{\beta}(j)\right)\right] d \mu_{\beta}(\phi) .
\end{aligned}
$$

One sees that the "observable", $e^{\varepsilon \phi(f)}$, can be eliminated by the change of variables (5.8).

Next, we apply Theorem 2.1, [replacing $1+z \cos \phi(j)$ by $1+z \cos \phi^{\prime}(j)$, with $\phi^{\prime}(j)$ $\left.=\phi(j)+\sigma_{\beta}(j)\right]$, to obtain

$$
\begin{aligned}
Z_{A}(\varepsilon f)= & e^{\left(\varepsilon^{2} \beta / 2\right)\left\langle f,(-\Delta)^{-1} f\right\rangle} \\
& \cdot\left\{\sum_{\mathscr{N} \in \mathscr{F}_{A}} c_{\mathscr{N}} \int \prod_{\varrho \in \mathscr{N}}\left[1+K(\varrho) \cos \left(\phi(\varrho)+\sigma_{\beta}(\varrho)\right)\right] d \mu_{\beta}(\phi)\right\},
\end{aligned}
$$

see Sect. 2.1, (2.3)-(2.8). To all terms on the right side of (5.13) we then apply Theorem 4.1, i.e.

$$
\begin{aligned}
Z_{\mathscr{N}}\left(\sigma_{\beta}\right) & \equiv \int \prod_{\varrho \in \mathscr{N}}\left[1+K(\varrho) \cos \left(\phi(\varrho)+\sigma_{\beta}(\varrho)\right)\right] d \mu_{\beta}(\phi) \\
& =\int \prod_{\varrho \in \mathscr{N}}\left[1+z(\beta, \varrho) \cos \left(\phi(\varrho)+\sigma_{\beta}(\varrho)\right)\right] d \mu_{\beta}(\phi),
\end{aligned}
$$

with

$$
z(\beta, \varrho) \leqq \exp [-(c \beta-b) \ln d(\varrho)],
$$

for some constants $c>0$ and $b<\infty$, provided $\beta>b / c$ [see (4.4), (4.5)].

We now choose $\beta$ so large that $z(\beta, \varrho)<1$, for all $\varrho \in \mathcal{N}$. Then

$$
\begin{aligned}
0 & <1+z(\beta, \varrho) \cos \left(\phi(\bar{\varrho})+\sigma_{\beta}(\varrho)\right) \\
& =\exp \ln \left[1+z(\beta, \varrho) \cos \left(\phi(\varrho)+\sigma_{\beta}(\varrho)\right)\right] .
\end{aligned}
$$

We define

$$
X=z(\beta, \varrho) \cos \phi(\bar{\varrho})
$$

and

$$
Y=z(\beta, \varrho)\left\{\cos \phi(\bar{\varrho})\left(\cos \sigma_{\beta}(\varrho)-1\right)-\sin \phi(\varrho) \sin \sigma_{\beta}(\varrho)\right\} .
$$

By a trigonometric identity, (5.16) and Taylor's theorem with remainder,

$$
\begin{aligned}
& 1+z(\beta, \varrho) \cos \left(\phi(\bar{\varrho})+\sigma_{\beta}(\varrho)\right)=\exp \ln [1+X+Y] \\
& \quad=(1+X) \exp \left[Y /(1+X)-(1 / 2) Y^{2} /(1+X+\theta Y)^{2}\right],
\end{aligned}
$$

for some $\theta \in(0,1)$. Let $z_{1}(\varrho)=z(\beta, \varrho)(1-z(\beta, \varrho))^{-1}$, and $[x]_{2 \pi}$ the value of $x \bmod 2 \pi$ between $-\pi$ and $\pi$.

Then

$$
z(\beta, \varrho) \cos \phi(\varrho)\left(\cos \sigma_{\beta}(\varrho)-1\right)(1+X)^{-1} \geqq-\left(z_{1}(\varrho) / 2\right)\left[\sigma_{\beta}(\varrho)\right]_{2 \pi}^{2} .
$$

We set

$$
S(\varrho ; \phi) \equiv z(\beta, \varrho) \sin \phi(\bar{\varrho}) \sin \sigma_{\beta}(\varrho)(1+X)^{-1},
$$


and note that, for $z(\beta, \varrho)<1, S(\varrho ; \phi)$ is bounded and is an odd function of $\phi$. Finally, if $\beta$ is so large that $z(\beta, \varrho)<1 / 4$, for all $\varrho \in \mathscr{N}$,

$$
Y^{2} /(1+X+\theta Y)^{2}<z_{2}(\varrho)\left[\sigma_{\beta}(\varrho)\right]_{2 \pi}^{2},
$$

where

$$
z_{2}(\varrho) \equiv(1+\pi) z(\beta, \varrho)^{2}(1-4 z(\beta, \varrho))^{-2}
$$

We now insert (5.17)-(5.20) into (5.14). This yields the lower bound

$$
\begin{aligned}
Z_{\mathscr{N}}\left(\sigma_{\beta}\right) \geqq & \exp \left\{-(1 / 2) \sum_{\varrho \in \mathcal{N}}\left(z_{1}(\varrho)+z_{2}(\varrho)\right)\left[\sigma_{\beta}(\varrho)\right]_{2 \pi}^{2}\right\} \\
& \cdot \int \prod_{\varrho \in \mathscr{N}} e^{S(\varrho ; \phi)}[1+z(\beta, \varrho) \cos \phi(\bar{\varrho})] d \mu_{\beta}(\phi) \\
\geqq & \exp \left\{-(1 / 2) \sum_{\varrho \in \mathcal{N}}\left(z_{1}(\varrho)+z_{2}(\varrho)\right)\left[\sigma_{\beta}(\varrho)\right]_{2 \pi}^{2}\right\} Z_{\mathscr{N}}
\end{aligned}
$$

with $Z_{\mathscr{N}} \equiv Z_{\mathscr{N}}(0)$. Recalling that $S(\varrho ; \phi)$ is odd in $\phi$, one sees that the second inequality in (5.21) follows from Jensen's inequality and the evenness in $\phi$ of the measure $\prod_{\varrho \in \mathcal{N}}[1+z(\beta, \varrho) \cos \phi(\bar{\varrho})] d \mu_{\beta}(\phi)$ which by $(5.15)$ is positive for $\beta$ sufficiently large.

In order to prove a lower bound on the functional $Z_{A}(\varepsilon f)$, it now suffices to exhibit a lower bound on

$$
\exp \left\{-(1 / 2) \sum_{\varrho \in \mathscr{N}}\left(z_{1}(\varrho)+z_{2}(\varrho)\right)\left[\sigma_{\beta}(\varrho)\right]_{2 \pi}^{2}\right\}
$$

which is independent of the ensemble $\mathscr{N}$, i.e. uniform in $\mathscr{N} \in \mathscr{F}_{A}$.

This is done as follows: Since all charge densities $\varrho \in \mathscr{N}$ are neutral,

$$
\sigma_{\beta}(\varrho)=\sum_{\mu}\left\{\sigma_{\beta}\left(p_{\mu}\right)-\sigma_{\beta}\left(n_{\mu}\right)\right\},
$$

where $p_{\mu} \in\{j: \varrho(j)=1\}, n_{\mu} \in\{j: \varrho(j)=-1\}$, and there are $A_{0}(\varrho) / 2$ terms in the sum on the right side of (5.22). Clearly

$$
\sigma_{\beta}\left(p_{\mu}\right)-\sigma_{\beta}\left(n_{\mu}\right)=\sum_{m=0}^{\ell-1}\left\{\sigma_{\beta}\left(j_{m}\right)-\sigma_{\beta}\left(j_{m+1}\right)\right\},
$$

where $j_{0}=p_{\mu}, j_{\ell}=n_{\mu}$, for some $\ell$, and $\left|j_{m}-j_{m+1}\right|=1$, for all $m=1, \ldots, \ell-1$. Since $p_{\mu}$ and $n_{\mu}$ belong to supp $\varrho, \ell \leqq 2 d(\varrho)$. We thus get

$$
\begin{aligned}
&\left|\sigma_{\beta}\left(p_{\mu}\right)-\sigma_{\beta}\left(n_{\mu}\right)\right| \leqq \max _{j \in D(\varrho)}\left|\left(\nabla \sigma_{\beta}\right)(j)\right| \cdot 2 d(\varrho) \\
&=\left|\left(\nabla \sigma_{\beta}\right)\left(j_{\varrho}\right)\right| \cdot 2 d(\varrho),
\end{aligned}
$$

for some $j_{\varrho} \in D(\varrho)$. (Here $\nabla$ is the finite difference gradient.) Thus

$$
\left|\sigma_{\beta}(\varrho)\right| \leqq A_{0}(\varrho) d(\varrho)\left|\left(\nabla \sigma_{\beta}\right)\left(j_{\varrho}\right)\right| .
$$


By the definitions of $z_{1}$ and $z_{2}$, we see that for $\beta$ sufficiently large there is a $\kappa$ such that

$$
\begin{aligned}
z_{1}(\varrho)+z_{2}(\varrho) & \leqq \operatorname{const} z(\beta, \varrho) \\
& <\exp \left[-\frac{\beta}{\kappa} \cdot \ln (d(\varrho))\right]
\end{aligned}
$$

for all $\varrho \in \mathscr{N}$ and all $\mathscr{N} \in \mathscr{F}$. Thus for $\beta \gg 1$

$$
\begin{aligned}
{\left[z_{1}(\varrho)+z_{2}(\varrho)\right]\left[\sigma_{\beta}(\varrho)\right]_{2 \pi}^{2} } & \leqq \operatorname{const} z(\beta, \varrho)\left[A_{0}(\varrho) d(\varrho)\right]^{2} \cdot\left|\left(\nabla \sigma_{\beta}\right)\left(j_{\varrho}\right)\right|^{2} \\
& \leqq \frac{\delta}{2 \beta}\left|\left(\nabla \sigma_{\beta}\right)\left(j_{\varrho}\right)\right|^{2} d(\varrho)^{-1}
\end{aligned}
$$

Note that

$$
\sum_{\varrho \in \mathcal{N}}\left|\left(\nabla \sigma_{\beta}\right)\left(j_{\varrho}\right)\right|^{2} d(\varrho)^{-1} \leqq \sum_{k \geqq 1} 2^{-k} \sum_{\substack{\varrho \in \mathcal{N} \\ 2^{k} \leqq d(\varrho) \leqq 2^{k+1}}}\left|\nabla \sigma_{\beta}\left(j_{\varrho}\right)\right|^{2} \leqq\left\|\nabla \sigma_{\beta}\right\|^{2} .
$$

In the last sum we have used the fact that $j_{\varrho}$ are distinct by (2.4). We therefore obtain

$$
\exp \left\{-(1 / 2) \sum_{\varrho \in \mathcal{N}}\left(z_{1}(\varrho)+z_{2}(\varrho)\right)\left[\sigma_{\beta}(\varrho)\right]_{2 \pi}^{2}\right\} \geqq e^{-(\delta / 2 \beta)\left\|\nabla \sigma_{\beta}\right\|_{2}^{2}} .
$$

By definition of $\sigma_{\beta}$, see (5.7),

$$
\left\|\nabla \sigma_{\beta}\right\|_{2}^{2}=\varepsilon^{2} \beta^{2}\left\langle f,(-\Delta)^{-1} f\right\rangle .
$$

Now, Eqs. (5.6), (5.13), and (5.14) yield

$$
Z_{\Lambda}(\varepsilon f)=e^{\left(\varepsilon^{2} \beta / 2\right)\left\langle f,(-\Delta)^{-1} f\right\rangle} \sum_{\mathscr{N} \in \mathscr{F}_{A}} c_{\mathscr{N}} Z_{\mathscr{N}}\left(\sigma_{\beta}\right),
$$

so that, using (5.21), (5.24), and (5.25), we get

But, $\sum_{\mathscr{N} \in \mathscr{F}_{A}} c_{\mathscr{N}} Z_{\mathscr{N}^{\prime}}=Z_{\Lambda}$, so that

$$
Z_{\Lambda}(\varepsilon f) \geqq \exp \left[\left(\varepsilon^{2} / 2\right)(1-\delta) \beta\left\langle f,(-\Delta)^{-1} f\right\rangle\right] \sum_{\mathscr{N} \in \mathscr{F}_{\Lambda}} c_{\mathscr{N}} Z_{\mathscr{N}} .
$$

$$
\left\langle e^{\varepsilon \phi(f)}\right\rangle_{\Lambda}(\beta, z)=Z_{\Lambda}^{-1} Z_{\Lambda}(\varepsilon f) \geqq e^{\left(\varepsilon^{2} / 2\right)(1-\delta) \beta\left\langle f,(-\Delta)^{-1} f\right\rangle}
$$

which completes the proof.

Remarks. If we set $f(j)=\delta_{j 0}-\delta_{j x}$ we get from Theorem 5.1

$$
\begin{aligned}
\left\langle(\phi(0)-\phi(x))^{2}\right\rangle_{\Lambda}(\beta, z) & \geqq 2(1-\delta) \beta[C(0,0)-C(0, x)] \\
& \approx(1-\delta) \beta \pi^{-1} \ln |x|,
\end{aligned}
$$

for large $|x|$, for all $\beta>\beta(\delta, z)$. This proves Theorem A, Sect. 1.3.

By polarization, inequality (5.4), Theorem 5.1, extends to complex-valued functions, $f$. Thus, in each translation-invariant thermodynamic limit, we obtain from (5.4) by Fourier transformation

$$
\begin{aligned}
\left\langle|\hat{\phi}(k)|^{2}\right\rangle(\beta, z) & \geqq(1-\delta) \beta \hat{C}(k) \\
& \approx(1-\delta) \beta k^{-2}, \text { for } k \neq 0 \text { small, }
\end{aligned}
$$


for all $\beta>\beta(\delta, z)$. As explained in Sect. 1.3, this implies that the Stillinger-Lovett sum rule is violated for sufficiently large $\beta$.

Next, we turn to our proof of Theorem B, Sect. 1.3. We propose to show that the fractional charge two-point correlation function $G_{\xi}(0, x)$, with $0<\xi<1$, satisfies the upper bound

$$
G_{\xi}(0, x) \leqq \operatorname{const}(1+|x|)^{-\delta(\beta, \xi)},
$$

for some constant $\delta(\beta, \xi)$ which is positive for all $\beta>\beta(\xi, z)$, and $\beta(\xi, z)$ is a finite constant, provided $\xi \in(0,1)$ and $z \leqq$ const $e^{\varepsilon \beta}$, with $\varepsilon<1 / 16$. The analogous lower bound (1.24) has been proven in [5, Sect. 3], and holds for all $\beta$ and $z \geqq 0$.

From (1.22), Sect. 1.3, we recall that, in a finite region $\Lambda$,

$$
\begin{aligned}
G_{\xi}(0, x) & =\left\langle e^{i \xi(\phi(0)-\phi(x))}\right\rangle_{\Lambda}(\beta, z) \\
& =\langle\cos [\xi(\phi(0)-\phi(x))]\rangle_{\Lambda}(\beta, z),
\end{aligned}
$$

by the $\phi \rightarrow-\phi$ symmetry of $\langle-\rangle_{A}(\beta, z)$.

By Theorem 2.3, Sect. 2.5,

$$
\begin{aligned}
G_{\xi}(0, x)= & Z_{A}^{-1} \sum_{\mathscr{N}} c_{\mathscr{N}} \int\left(\cos \phi\left(\xi \varrho_{0}\right)+K\left(\varrho^{\mathcal{N}}\right) \cos \phi\left(\xi \varrho_{0}+\varrho^{\mathcal{N}}\right)\right) \\
& \cdot \prod_{\varrho \in \mathscr{N}}(1+K(\varrho) \cos \phi(\varrho)) d \mu_{\beta}(\phi)
\end{aligned}
$$

where $\varrho_{0}(j)=\delta_{j 0}-\delta_{j x}$. It is obvious that $\varrho_{0}$ satisfies Condition (2.28), Sect. 2.5. Thus, for $0<\xi<1$, we can apply Theorem 4.1, see also (4.57), and conclude

$$
\begin{aligned}
G_{\xi}(0, x)= & Z_{A}^{-1} \sum_{\mathcal{N}} c_{\mathscr{N}} \int\left(z\left(\beta, \xi \varrho_{0}\right) \cos \phi\left(\xi \bar{\varrho}_{0}\right)+z\left(\beta, \xi \varrho_{0}+\varrho^{\mathscr{N}}\right)\right. \\
& \left.\cdot \cos \phi\left(\overline{\xi \varrho_{0}+\varrho^{\mathcal{N}}}\right)\right) \prod_{\varrho \in \mathcal{N}}(1+z(\beta, \varrho) \cos \phi(\bar{\varrho})) d \mu_{\beta}(\phi) .
\end{aligned}
$$

By (4.2) and (4.4)

$$
z(\beta, \varrho) \leqq \exp [-(c \beta-b) \ln d(\varrho)]
$$

if $z \leqq$ const $e^{\varepsilon \beta}$, with $\varepsilon<1 / 16$, and $\beta>b / c$, for some constants $c>0$ and $b<\infty$ (depending on $z$ ). Furthermore,

$$
0<z\left(\beta, \xi \varrho_{0}\right) \leqq \exp \left[-c^{\prime} \xi^{2} \beta \ln d\left(\varrho_{0}\right)\right],
$$

for some positive constant $c^{\prime}<1 / 2 \pi$.

Let $\eta=\min (\xi, 1-\xi)$, and let $c$ and $b$ be the same constants as in (5.31). The bounds on $z\left(\beta, \xi \varrho_{0}+\varrho^{\mathscr{N}}\right)$ are

$$
0<z\left(\beta, \xi \varrho_{0}+\varrho^{\mathscr{N}}\right) \leqq \exp \left[-\left(c \eta^{2} \beta-b\right) \ln d\left(\xi \varrho_{0}+\varrho^{\mathscr{N}}\right)\right]
$$

for $\beta>b / c \eta^{2}$.

The proof of (5.32) is really a simpler variant of the one of Lemma 4.4. The bound (5.33), proven in Theorem 4.1, see (4.58), is quite crude. In the form given here, it requires $\beta>0\left(\eta^{-2}\right)$ which is a somewhat awkward condition. It can be improved by going through the renormalization of $\cos \phi\left(\xi \varrho_{0}+\varrho^{\mathcal{N}}\right)$ more carefully; see Lemma 4.4. However, our methods only prove that

$$
z\left(\beta, \xi \varrho_{0}+\varrho^{\mathscr{N}}\right) \leqq \exp \left[-c^{\prime \prime} \ln d\left(\xi \varrho_{0}+\varrho^{\mathscr{N}}\right)\right],
$$

for some constant $c^{\prime \prime}>0$, if $\beta>0\left(\eta^{-2}\right)$. 
By (5.31),

$$
z(\beta, \varrho)<1, \text { for all } \varrho \in \mathscr{N} \text { and all } \mathscr{N}
$$

if $\beta>b / c$, so that

$$
\prod_{\varrho \in \mathscr{N}}(1+z(\beta, \varrho) \cos \phi(\bar{\varrho})) d \mu_{\beta}(\phi)
$$

is a positive measure. Since $z\left(\beta, \xi \varrho_{0}\right)$ and $z\left(\beta, \xi \varrho_{0}+\varrho^{\mathcal{N}}\right)$ are positive, for all $\beta, \xi$ and $z>0$, we therefore obtain from (5.30) and from the trivial inequality $|z \cos \alpha| \leqq z$, for $z>0$,

$$
\begin{aligned}
G_{\xi}(0, x) \leqq & Z_{\Lambda}^{-1} \sum_{\mathscr{N}} c_{\mathscr{N}}\left[z\left(\beta, \xi \varrho_{0}\right)+z\left(\beta, \xi \varrho_{0}+\varrho^{\mathscr{N}}\right)\right] \\
& \cdot \int \prod_{\varrho \in \mathscr{N}}(1+z(\beta, \varrho) \cos \phi(\varrho)) d \mu_{\beta}(\phi) \\
\leqq & \max _{\mathscr{N}}\left[z\left(\beta, \xi \varrho_{0}\right)+z\left(\beta, \xi \varrho_{0}+\varrho^{\mathscr{N}}\right)\right] \\
& \cdot Z_{\Lambda}^{-1} \sum_{\mathscr{N}} c_{\mathscr{N}} \int \prod_{\varrho \in \mathscr{N}}(1+z(\beta, \varrho) \cos \phi(\varrho)) d \mu_{\beta}(\phi),
\end{aligned}
$$

provided $\beta>b / c$. By Corollary 2.4, Sect. 2.5, and Theorem 4.1

$$
\begin{aligned}
Z_{A} & =\sum_{\mathcal{N}} c_{\mathcal{N}} \int\left(1+K\left(\varrho^{\mathscr{N}}\right) \cos \phi\left(\varrho^{\mathscr{N}}\right)\right) \prod_{\varrho \in \mathscr{N}}(1+K(\varrho) \cos \phi(\varrho)) d \mu_{\beta}(\phi) \\
& =\sum_{\mathscr{N}} c_{\mathcal{N}} \int\left(1+z\left(\beta, \varrho^{\mathscr{N}}\right) \cos \phi\left(\overline{\varrho^{\mathcal{N}}}\right) \prod_{\varrho \in \mathscr{N}}(1+z(\beta, \varrho) \cos \phi(\bar{\varrho})) d \mu_{\beta}(\phi),\right.
\end{aligned}
$$

where the renormalized charge densities $\bar{\varrho}$, and the renormalized activities, $z(\beta, \varrho)$, are identical to the ones in Eqs. (5.30), for all $\varrho \in \mathscr{N}$. This last assertion follows from the simple fact that, by Theorem 2.3 and Corollary 2.4, the renormalization transformation of $\prod_{\varrho \in \mathcal{N}}(1+K(\varrho) \cos \phi(\varrho))$ may be chosen to be independent of $\xi$. Since the characteristic functional, (i.e. the Fourier transform) of $d \mu_{\beta}(\phi)$ is positive,

$$
\begin{aligned}
& \int\left(1+z\left(\beta, \varrho^{\mathscr{N}}\right) \cos \phi\left(\overline{\varrho^{\mathcal{N}}}\right)\right) \prod_{\varrho \in \mathscr{N}}(1+z(\beta, \varrho) \cos \phi(\bar{\varrho})) d \mu_{\beta}(\phi) \\
& \quad \geqq \int \prod_{\varrho \in \mathscr{N}}(1+z(\beta, \varrho) \cos \phi(\bar{\varrho})) d \mu_{\beta}(\phi),
\end{aligned}
$$

for all $\mathscr{N}$. Thus, using (5.34),

$$
\begin{aligned}
G_{\xi}(0, x) \leqq & \max _{\mathscr{N}}\left[z\left(\beta, \xi \varrho_{0}\right)+z\left(\beta, \xi \varrho_{0}+\varrho^{\mathscr{N}}\right)\right] \\
& \cdot Z_{\Lambda}^{-1} \sum_{\mathcal{N}} c_{\mathcal{N}} \int\left(1+z\left(\beta, \varrho^{\mathscr{N}}\right) \cos \phi\left(\overline{\varrho^{\mathscr{N}}}\right)\right) \\
& \cdot \prod_{\varrho \in \mathscr{N}}(1+z(\beta, \varrho) \cos \phi(\bar{\varrho})) d \mu_{\beta}(\phi) \\
= & \max _{\mathscr{N}}\left[z\left(\beta, \xi \varrho_{0}\right)+z\left(\beta, \xi \varrho_{0}+\varrho^{\mathscr{N}}\right)\right] .
\end{aligned}
$$

Finally, we note that

$$
d\left(\xi \varrho_{0}+\varrho^{\mathscr{N}}\right) \geqq d\left(\xi \varrho_{0}\right)=d\left(\varrho_{0}\right)=|x|,
$$


for $0<\xi<1$. It follows therefore from (5.32) and (5.33) that for $\beta>b / c \eta^{2}$

$$
G_{\xi}(0, x)<2 \exp \left[-\left(c \eta^{2} \beta-b\right) \ln |x|\right]
$$

which yields (5.27).

\subsection{General Coulomb Gases, Villain Model and Discrete Gaussian}

We now extend the arguments in Sect. 5.1 to general Coulomb gases with charge distributions $d \lambda(q)$ satisfying Condition $\lambda$, Sect. 1.2. As we already noticed in Sect. 1.4, the dual of the Villain model, identical to the discrete Gaussian, is included in that class. For this model we prove Theorems C, D stated in Sect. 1.4. Our first task is to extend Theorem 2.1 to the general class of Coulomb gases characterized by Condition $\lambda$, Sect. 1.2. Thus we consider a general a priori distribution $d \lambda(q)$ with $\operatorname{supp} \lambda \subseteq \mathbb{Z}$ and $\lambda(q)=\lambda(-q)$. It is not necessary to assume that $\lambda$ is a positive measure, but the growth condition

$$
|\lambda(q)| \leqq \text { const } e^{(\alpha+\varepsilon \beta) q^{2}}, \quad \varepsilon<1 / 16,
$$

is essential. Fourier transformation yields

$$
\hat{\lambda}(\phi)=1+\sum_{q=1}^{\infty} 2 \lambda(q) \cos (q \phi) .
$$

We now choose a sequence, $\left\{\zeta_{q}\right\}_{q=1}^{\infty}$, of positive numbers with

$$
\sum_{q=1}^{\infty} \zeta_{q}=1, \text { and }\left|\zeta_{q}\right|^{-1}<\mathrm{const} e^{\alpha^{\prime} q^{2}}
$$

for some finite, positive $\alpha^{\prime}$. (An explicit choice of $\left\{\zeta_{q}\right\}$ is made later.) We then get

$$
\hat{\lambda}(\phi)=\sum_{q=1}^{\infty} \zeta_{q}(1+z(q) \cos (q \phi))
$$

where $z(q)=2 \zeta_{q}^{-1} \lambda(q)$. Hence

$$
\prod_{j \in \Lambda} \hat{\lambda}(\phi(j)) \leqq \sum_{q_{\Lambda}} \zeta\left(q_{\Lambda}\right) \prod_{j \in \Lambda}\left[1+z\left(q_{j}\right) \cos \left(q_{j} \phi(j)\right)\right],
$$

with

$$
q_{\Lambda}=\left\{q_{j}\right\}_{j \in \Lambda}, \quad \zeta\left(q_{\Lambda}\right) \equiv \prod_{j \in \Lambda} \zeta_{q_{j}} .
$$

For each term on the right side of (5.39) the following result closely related to Theorem 2.1 holds.

Theorem 5.2. There exists a family $\mathscr{F}_{q_{A}}$, of ensembles, $\mathscr{N}$, such that

$$
\prod_{j \in \Lambda}\left[1+z\left(q_{j}\right) \cos \left(q_{j} \phi(j)\right)\right]=\sum_{\mathcal{N} \in \mathscr{F}_{q_{A}}} c_{\mathscr{N}}^{\prime} \prod_{\varrho \in \mathcal{N}}(1+K(\varrho) \cos \phi(\varrho)),
$$

where $c_{\mathscr{N}}^{\prime}>0$, and $\varrho$ is a charge density with the property that $\varrho(j)= \pm q_{j}$, for all $j \in \Lambda$, for all $\mathscr{N} \in \mathscr{F}_{q_{\Lambda}}$, and each $\mathcal{N} \in \mathscr{F}_{q_{\Lambda}}$ has properties a)-c) stated in Theorem 2.1, for all $q_{\Lambda}$. Moreover,

$$
K(\varrho)=\prod_{j \in \operatorname{supp} \varrho} z(|\varrho(j)|) e^{S(\varrho)},
$$

with $S(\varrho) \leqq C A(\varrho), A(\varrho) \equiv \sum_{k=0}^{n(\varrho)} A_{k}(\varrho)$, where $C, n(\varrho)$ and $A_{k}(\varrho)$ are as in Theorem 2.1 . 
Remark. The proof of Theorem 5.2 is identical to the one of Theorem 2.1, except that, in the last step of the recursion $(2.21),(2.22), z^{A_{0}(\varrho)}$ must be replaced by the factor $\prod_{j \in \text { supp } \varrho} z(|\varrho(j)|)$. From (5.39) and Theorem 5.2 we now obtain

$$
\begin{aligned}
\prod_{j \in \Lambda} \hat{\lambda}(\phi(j)) & =\sum_{q_{\Lambda}} \zeta\left(q_{\Lambda}\right) \sum_{\mathscr{N} \in \mathscr{F}_{q_{A}}} c_{\mathscr{N}}^{\prime} \prod_{\varrho \in \mathscr{N}}(1+K(\varrho) \cos \phi(\varrho)) \\
& =\sum_{\mathscr{N} \in \mathscr{F}_{A}} c_{\mathscr{N}} \prod_{\varrho \in \mathscr{N}}(1+K(\varrho) \cos \phi(\varrho))
\end{aligned}
$$

with

$$
\mathscr{F}_{\Lambda}=\bigcup_{q_{\Lambda}} \mathscr{F}_{q_{\Lambda}}, c_{\mathcal{N}}=\zeta\left(q_{\Lambda}\right) c_{\mathscr{N}}^{\prime}, \quad \text { for } \quad \mathcal{N} \in \mathscr{F}_{q_{\Lambda}} .
$$

It is clear that Theorem 2.3 and Corollary 2.4 extend to the more general situation considered here, as well.

Next, we rephrase the results of Sects. 3 and 4 for the models studied here. By Theorem 4.1 and its proof, see Sect. 4.4,

$$
\begin{aligned}
\int & \prod_{\varrho \in \mathcal{N}}[1+K(\varrho) \cos (\phi(\varrho)+\sigma(\varrho))] d \mu_{\beta}(\phi) \\
& =\int \prod_{\varrho \in \mathcal{N}}[1+z(\beta, \varrho) \cos (\phi(\bar{\varrho})+\sigma(\varrho))] d \mu_{\beta}(\phi),
\end{aligned}
$$

where

$$
z(\beta, \varrho)=K(\varrho) \exp \left[-\beta E_{\text {loc }}(\varrho, \mathscr{N})\right],
$$

and by inequality (4.58), Sect. 4.4,

$$
E_{\mathrm{loc}}(\varrho, \mathscr{N}) \geqq(1 / 2) E_{0}(\varrho)+D \sum_{k=1}^{\infty}\left|\mathscr{S}_{k}^{\prime}(\varrho)\right|
$$

with

$$
E_{0}(\varrho)=(1 / 8) \sum_{j} \varrho(j)^{2}
$$

[see (4.17)-(4.19), Sect. 4.2]. By Theorem 5.1,

$$
|K(\varrho)| \leqq \prod|z(|\varrho(j)|)| \exp [C A(\varrho)] .
$$

In Sect. 3 we have shown that

$$
A(\varrho) \leqq E A_{0}(\varrho)+F \sum_{k=0}^{\infty}\left|\mathscr{S}_{k}^{\prime}(\varrho)\right|,
$$

for some finite constants $E$ and $F$; see inequality (3.17). Thus

$$
\begin{aligned}
|z(\beta, \varrho)| \leqq & \prod_{j}|z(|\varrho(j)|)| \exp \left[-\left(c^{\prime} \beta-b^{\prime}\right) \varrho(j)^{2}\right] \\
& \cdot \exp [-(c \beta-b) A(\varrho)],
\end{aligned}
$$

for some constants $c^{\prime}>0, b^{\prime}<\infty, c>0$ and $b<\infty$, provided $\beta>\max \left(b^{\prime} / c^{\prime}, b / c\right)$. The constant $c^{\prime}<1 / 16$ can be chosen arbitrarily close to $1 / 16$. If

$$
|\lambda(q)| \leqq \text { const } \exp \left[(\alpha+\varepsilon \beta) q^{2}\right] \text {, with } \quad \varepsilon<1 / 16,
$$


we conclude that

$$
|z(|\varrho(j)|)| \exp \left[-\left(c^{\prime} \beta-b^{\prime}\right) \varrho(j)^{2}\right] \leqq \exp \left[-\left(c^{\prime \prime} \beta-b^{\prime \prime}\right) \varrho(j)^{2}\right],
$$

for some $c^{\prime \prime}>0$ and $b^{\prime \prime}$, by choosing for example $\zeta_{q}=$ const $e^{-\delta q^{2}}$ and optimizing in $\delta$.

We have now proven

Theorem 5.3. If $d \lambda$ satisfies (5.47), then there exists a finite constant $\beta(\lambda)$ such that for all $\beta \geqq \beta(\lambda)$

$$
|z(\beta, \varrho)| \leqq \exp \left[-\left(c^{\prime \prime} \beta-b^{\prime \prime}\right)\|\varrho\|_{2}^{2}\right] \exp [-(c \beta-b) A(\varrho)]
$$

where $\|\varrho\|_{2}^{2}=\sum_{j} \varrho(j)^{2}$; and $A(\varrho) \geqq$ const $\ln d(\varrho)$.

This result also applies to the situation described in Theorem 2.3 and Corollary 2.4 , (i.e. in the presence of fractional charges), and yields upper bounds on $z\left(\beta, \xi \varrho_{0}\right)$ and $z\left(\beta, \xi \varrho_{0}+\varrho^{\mathcal{N}}\right)$ : It suffices to replace $c$ by $c \eta^{2}, \eta=\min (\xi, 1-\xi)$; see (5.30)-(5.33). In conclusion, the proofs of Theorems $\mathrm{A}$ and $\mathrm{B}$ and the lower bound (5.5) on $\left\langle e^{\varepsilon \phi(f)}\right\rangle^{\phi}(\beta, \lambda)$ for the general Coulomb gases considered here are completely analogous to the case of the hard core gas, Sect. 5.1, if one uses Theorems 5.2 and 5.3. [The only estimate that requires some additional thought in the proof of (5.5) is (5.23) which we reconsider below.]

From (1.22) and (1.38) we see that Theorem D for the Villain model is a special case of Theorem B for a general Coulomb gas. We are thus left with proving Theorem $\mathrm{C}$ for the Villain model. Recall from Sect. 1.4 that

$$
\left\langle e^{i \xi\left(\theta_{0}-\theta_{x}\right)}\right\rangle(\beta)=\left\langle\hat{D}_{0 x}^{\xi}\right\rangle^{*}(\beta), \quad \xi \in \mathbb{Z},
$$

where

$$
\hat{D}_{0 x}^{\xi}=\exp \left[\beta^{-1} \xi \phi\left(\partial_{2} f^{x}\right)-(2 \beta)^{-1} \xi^{2}|x|\right]
$$

with

$$
f^{x}\left(j_{1}, j_{2}\right)= \begin{cases}1, & 1 \leqq j_{1} \leqq x, \quad j_{2}=0 \\ 0, & \text { otherwise, }\end{cases}
$$

and $\partial_{\alpha}, \alpha=1,2$, is the $\alpha^{\text {th }}$ component of the finite difference gradient [see (1.36) and (1.37) in Sect. 1.4]. In particular,

$$
\left\langle\mathbf{S}_{0} \cdot \mathbf{S}_{x}\right\rangle(\beta)=\left\langle e^{i\left(\theta_{0}-\theta_{x}\right)}\right\rangle(\beta)=\left\langle\hat{D}_{0 x}^{1}\right\rangle^{*}(\beta) .
$$

We propose to prove

Theorem 5.4. For the Villain model there exists some finite constant $\beta_{0}$ such that for $\beta>\beta_{0}$

$$
\left\langle\hat{D}_{0 x}^{\xi}\right\rangle^{*}(\beta) \geqq \text { const } \exp \left[-\left(\xi^{2} / 2 \pi \beta^{\prime}\right) \ln (1+|x|)\right], \quad \xi \in \mathbb{Z},
$$

for some positive $\beta^{\prime}=\beta^{\prime}(\beta) \rightarrow \infty$, as $\beta \rightarrow \infty$.

Remarks. 1. Theorem C, Sect. 1.4, is the special case of Theorem 5.4 in which $\xi=1$. 
2. If $\lambda$ is the a priori distribution of a general Coulomb gas satisfying the growth condition (5.36) then there exists some finite $\beta(\lambda)$ such that for all $\beta>\beta(\lambda)$

$$
\left\langle\hat{D}_{0 x}^{\xi}\right\rangle^{\phi}(\beta, \lambda) \geqq \text { const } \exp \left[-\left(\xi^{2} / 2 \beta^{\prime}\right) \ln (1+|x|)\right],
$$

for $\xi \in 2 \pi \mathbb{Z}$, for some $\beta^{\prime}=\beta^{\prime}(\beta, \lambda)>0$.

(The modified condition on $\xi, \xi \in 2 \pi \mathbb{Z}$, corresponds to the rescaling $\phi \rightarrow \phi^{\prime}$ $=2 \pi \phi$ which relates the dual of the Villain model to a Coulomb gas; see Sect. 1.4.) Clearly Theorem 5.4 is a special case of (5.51).

Proof of (5.51). By (5.40) and the definition of $\langle-\rangle_{A}^{\phi}(\beta, \lambda)$

$$
\begin{aligned}
\left\langle\hat{D}_{0 x}^{\zeta}\right\rangle_{\Lambda}^{\phi}(\beta, \lambda) & =Z_{A}^{-1} \int \hat{D}_{0 x}^{\xi}(\phi) \prod_{j \in A} \hat{\lambda}(\phi(j)) d \mu_{\beta}(\phi) \\
& =Z_{A}^{-1} \sum_{\mathscr{N} \in \mathscr{F}_{A}} c_{\mathscr{N}} \int \hat{D}_{0 x}^{\xi}(\phi) \prod_{\varrho \in \mathcal{N}}(1+K(\varrho) \cos \phi(\varrho)) d \mu_{\beta}(\phi) .
\end{aligned}
$$

In each term on the right side of (5.52) we now make the following real change of variables:

where

$$
\left.\begin{array}{c}
\phi(j) \rightarrow \phi(j)+\sigma(j), \\
\sigma(j) \equiv \xi\left(C * \partial_{2} f^{x}\right)(j)=\xi \sum_{k} C(j-k)\left(\partial_{2} f^{x}\right)(k),
\end{array}\right\}
$$

and $f^{x}$ has been defined in (5.49). Using identities (5.9)-(5.11) and the definition (5.49) of $\hat{D}_{0 x}^{\xi}$ we see that, after the change of variables (5.53), $\hat{D}_{0 x}^{\xi}$ is cancelled, and we get

$$
Z_{\Lambda}\left\langle\hat{D}_{0 x}^{\zeta}\right\rangle_{\Lambda}^{\phi}(\beta, \lambda)=e^{G(x)} \sum_{\mathcal{N}} c_{\mathscr{N}} \int \prod_{\varrho \in \mathcal{N}}[1+K(\varrho) \cos (\phi(\varrho)+\sigma(\varrho))] d \mu_{\beta}(\phi),
$$

where $e^{G(x)}$ represents the spin wave or Gaussian contribution to $\left\langle\hat{D}_{0 x}^{\xi}\right\rangle_{A}^{\phi}(\beta, \lambda)$ $\left(=\left\langle e^{i \xi\left(\theta_{0}-\theta_{x}\right)}\right\rangle_{A}(\beta)\right.$, in the case of the Villain model). More explicitly

$$
\begin{aligned}
G(x) & =(2 \beta)^{-1}\left\{\langle\sigma, \Delta \sigma\rangle-\xi^{2}|x|+2 \xi\left\langle\partial_{2} f^{x}, \sigma\right\rangle\right\} \\
& =(2 \beta)^{-1}\left\{-\langle\sigma, \Delta \sigma\rangle-\xi^{2}|x|\right\} .
\end{aligned}
$$

By definition of $f^{x}$ and $\sigma$,

$$
\begin{aligned}
-\langle\sigma, \Delta \sigma\rangle & =\xi^{2}\left\langle\partial_{2} f^{x}, C * \partial_{2} f^{x}\right\rangle \\
& =\xi^{2}\left\langle f^{x}, \partial_{2}^{*} \partial_{2} C * f^{x}\right\rangle \\
& =\xi^{2}\left\langle f^{x}, f^{x}\right\rangle-\xi^{2}\left\langle\partial_{1} f^{x}, C * \partial_{1} f^{x}\right\rangle \\
& =\xi^{2}|x|-2 \xi^{2}\{C(0)-C(x)\},
\end{aligned}
$$

where we have used that

$$
\left(\partial_{1} f^{x}\right)(j)=\delta_{j 0}-\delta_{j x}
$$

Thus

$$
G(x)=-\beta^{-1} \xi^{2}[C(0)-C(x)] \approx-\left(\xi^{2} / 2 \pi \beta\right) \ln |x|,
$$


for $|x|$ large. Next, we apply the renormalization transformation (5.41) and obtain

$$
\begin{aligned}
Z_{\mathcal{N}}(\sigma) & \equiv \int \prod_{\varrho \in \mathscr{N}}[1+K(\varrho) \cos (\phi(\varrho)+\sigma(\varrho))] d \mu_{\beta}(\phi) \\
& \equiv \int \prod_{\varrho \in \mathcal{N}}[1+z(\beta, \varrho) \cos (\phi(\varrho)+\sigma(\varrho))] d \mu_{\beta}(\phi)
\end{aligned}
$$

By Theorem 5.3, there exists some finite $\beta_{1}(\lambda)$ such that

$$
\max _{\varrho \in \mathcal{N}}|z(\beta, \varrho)|<1 / 4, \quad \text { for all } \mathscr{N} \in \mathscr{F}_{\Lambda},
$$

provided $\beta>\beta_{1}(\lambda)$. As in (5.21) we then obtain the lower bound

$$
Z_{\mathcal{N}}(\sigma) \geqq \exp \left\{-(1 / 2) \sum_{\varrho \in \mathcal{N}}\left(z_{1}(\varrho)+z_{2}(\varrho)\right)[\sigma(\varrho)]_{2 \pi}^{2}\right\} Z_{\mathscr{N}},
$$

for $\beta>\beta_{1}(\lambda)$, where

$$
z_{1}(\varrho)=|z(\beta, \varrho)|(1-|z(\beta, \varrho)|)^{-1}
$$

and

$$
z_{2}(\varrho)=(1+\pi)|z(\beta, \varrho)|^{2}(1-4|z(\beta, \varrho)|)^{-2} .
$$

The proof of (5.58) is identical to the one of (5.21), see (5.16)-(5.20). We are thus left with estimating $\sum_{\varrho \in \mathcal{N}}\left(z_{1}(\varrho)+z_{2}(\varrho)\right)[\sigma(\varrho)]_{2 \pi}^{2}$. For this purpose we rewrite $\sigma(\varrho)$, using the neutrality of $\varrho$, as in (5.22):

$$
\sigma(\varrho)=\sum_{\mu} \sigma\left(p_{\mu}\right)-\sigma\left(n_{\mu}\right)
$$

for some sites $p_{\mu}$ and $n_{\mu}$ in supp $\varrho$. There are $\frac{1}{2}\|\varrho\|_{1}=\frac{1}{2} \sum_{j}|\varrho(j)|$ terms in the sum on the right side of (5.59). We now claim that

$$
\begin{aligned}
\sigma\left(p_{\mu}\right)-\sigma\left(n_{\mu}\right)= & \sum_{j}\left\{\varepsilon_{1}(j)\left(\partial_{1} \sigma\right)(j)+\varepsilon_{2}(j)\left(\partial_{2}^{*} \sigma\right)(j)\right\} \\
= & \sum_{j}\left\{\varepsilon_{1}(j) \xi\left[\partial_{2}(C(j)-C(j-x))\right]\right. \\
& \left.+\varepsilon_{2}(j) \xi\left[f^{x}(j)+\partial_{1}\left(C\left(j^{\prime}\right)-C\left(j^{\prime}-x\right)\right)\right]\right\},
\end{aligned}
$$

where $j^{\prime}$ is the neighbor of $j$ in the positive 1 -direction, $\varepsilon_{\kappa}(\mathrm{j})=0, \pm 1$, $\operatorname{supp} \varepsilon_{\kappa} \subseteq D(\varrho)$, for $\kappa=1,2$, and

$$
\sum_{j}\left\{\left|\varepsilon_{1}(j)\right|+\left|\varepsilon_{2}(j)\right|\right\} \leqq 2 d(\varrho) .
$$

The second equation in (5.60) follows from the identities

$$
\left.\begin{array}{rl}
\xi^{-1} \partial_{1} \sigma & =\partial_{1} C * \partial_{2} f^{x}=\partial_{2} C * \partial_{1} f^{x}=\partial_{2}[C(j)-C(j-x)], \\
\xi^{-1} \partial_{2}^{*} \sigma(j) & =-\Delta C * f^{x}(j)-\partial_{1} \partial_{1}^{*} C * f^{x}(j) \\
& =f^{x}(j)+\partial_{1}\left[C\left(j^{\prime}\right)-C\left(j^{\prime}-x\right)\right],
\end{array}\right\}
$$

and we have used (5.55). 
Combining (5.60) and (5.61) yields

$$
\left|\left[\sigma\left(p_{\mu}\right)-\sigma\left(n_{\mu}\right)\right]_{2 \pi}\right|<2 d(\varrho) \xi \max _{j \in \text { supp }_{1} \varrho}|\nabla(C(\mathrm{j})-\mathrm{C}(\mathrm{j}-\mathrm{x}))| .
$$

We choose $j_{\varrho} \in \operatorname{supp}_{1} \varrho$ so that

$$
\left|\nabla\left(C\left(j_{\varrho}\right)-C\left(j_{\varrho}-x\right)\right)\right|=\max _{j \in \sup _{1} \varrho}|\nabla(C(j)-C(j-x))| .
$$

Thus

$$
\left|[\sigma(\varrho)]_{2 \pi}\right| \leqq\|\varrho\|_{1} d(\varrho) \xi\left|\nabla\left(C\left(j_{\varrho}\right)-C\left(j_{\varrho}-x\right)\right)\right| .
$$

By Theorem 5.3 and (5.63) there exists a finite $\beta(\lambda, \delta)$ such that for all $\beta>\beta(\lambda, \delta)$

$$
\begin{aligned}
\left(z_{1}(\varrho)+z_{2}(\varrho)\right)[\sigma(\varrho)]_{2 \pi}^{2} \leqq & \operatorname{const} \exp \left[-\left(c^{\prime \prime} \beta-b^{\prime \prime}\right)\|\varrho\|_{2}^{2}\right] \cdot\|\varrho\|_{1}^{2} \\
& \cdot \exp \left[-\left(c^{\prime \prime \prime} \beta-b^{\prime \prime \prime}\right) \ln d(\varrho)\right] d(\varrho)^{2} \xi^{2} \mid \nabla\left(C\left(j_{\varrho}\right)-\left.C\left(j_{\varrho}-x\right)\right|^{2}\right. \\
\leqq & \xi^{2}(\delta / \beta) \mid \nabla\left(C\left(j_{\varrho}\right)-\left.C\left(j_{\varrho}-x\right)\right|^{2},\right.
\end{aligned}
$$

for all $\varrho \in \mathscr{N}$ and all $\mathscr{N} \in \mathscr{F}_{\Lambda}$.

Thus

$$
\begin{aligned}
\sum_{\varrho \in \mathcal{N}}\left(z_{1}(\varrho)+z_{2}(\varrho)\right)[\sigma(\varrho)]_{2 \pi}^{2} & \leqq \xi^{2}(\delta / \beta)\|\nabla(C(\cdot)-C(\cdot-x))\|_{2}^{2} \\
& =\xi^{2}(\delta / \beta) 2[C(0)-C(x)] .
\end{aligned}
$$

By (5.58) we therefore have

$$
Z_{\mathscr{N}}(\sigma) \geqq \exp \left[-\beta^{-1} \xi^{2} \delta(C(0)-C(x))\right] Z_{\mathscr{N}}
$$

which, together with (5.54) and (5.56) completes the proof of (5.51), for $\delta<1$ and $\beta>\beta(\lambda, \delta)$. This completes our analysis of lattice Coulomb gases, the Villain model and the discrete Gaussian.

\section{Kosterlitz-Thouless Transition in the Two-Dimensional Plane Rotator}

In this section we complete our analysis of the Kosterlitz-Thouless transition in the two-dimensional plane rotator model. In particular we prove Theorem C, Sect. 1.4. Definitions and notation are as in Sect. 1.4. Our purpose is to prove that there exists some finite $\beta_{0}$ such that, for all $\beta>\beta_{0}$,

$$
\left\langle e^{i \xi\left(\theta_{0}-\theta_{x}\right)}\right\rangle(\beta)=\left\langle\hat{D}_{0 x}^{\xi}\right\rangle^{*}(\beta) \geqq \text { const } \exp \left[-\left(\xi^{2} / 2 \pi \beta^{\prime}\right) \ln (1+|x|)\right],
$$

$0 \neq \xi \in \mathbb{Z}$, for some $\beta^{\prime}=\beta^{\prime}(\beta)>0$ tending to $\infty$, as $\beta \rightarrow \infty$. It is known from a standard high temperature expansion that for sufficiently small $\beta$

$$
\left\langle e^{i \xi\left(\theta_{0}-\theta_{x}\right)}\right\rangle(\beta) \leqq \text { const } \exp [-m(\beta)|x|],
$$

for some $m(\beta)>0$.

Thus, the lower bound (6.1) establishes the existence of a transition in this model. For $\xi=1$

$$
\left\langle e^{i\left(\theta_{0}-\theta_{x}\right)}\right\rangle(\beta)=\left\langle\mathbf{S}_{0} \cdot \mathbf{S}_{x}\right\rangle(\beta),
$$


see (1.50) and (1.58), Sect. 1.4. Therefore Theorem C, Sect. 1.4, is really a special case of (6.1).

Proof of (6.1). Our proof is closely related to the one given for the Villain model in Sect. 5.2; see Theorem 5.4. The first step consists in passing to the dual model and rewriting it as a perturbation of a zero-mass measure by "irrelevant (for large $\beta$ ) operators", For this purpose we introduce a function $I_{\beta}(\phi)$ which is complex analytic and non-zero in the strip $|\operatorname{Im} \phi| \leqq \beta / 2$ and satisfies the following conditions [see also Condition (4.10), Sect. 4.2]:

a) For integer $n$

$$
I_{\beta}(n)=\frac{1}{2 \pi} \int_{0}^{2 \pi} e^{\beta \cos \theta} e^{\mathrm{in} \theta} d \theta
$$

(b) $I_{\beta}(\phi)$ is even and positive on the real axis and integrable.

(c)

$$
\left|I_{\beta}(\phi+i a) / I_{\beta}(\phi)\right| \leqq e^{g(a) / \beta}, \quad \phi \in \mathbb{R},
$$

where

(d)

$$
\begin{gathered}
0 \leqq g(a) \leqq\left\{\begin{array}{l}
\text { const } a^{2},|a| \leqq 1 \\
\text { const } e^{2 \pi|a|}, 1 \leqq|a| \leqq \beta / 2
\end{array}\right. \\
\left|\frac{d^{m}}{d \phi^{m}} \log \left(I_{\beta}(\phi+i a) / I_{\beta}(\phi)\right)\right| \leqq C \beta^{-1} e^{2 \pi|a|},
\end{gathered}
$$

for $m=1,2$, and $|a| \leqq \beta / 2$, uniformly in $\phi \in \mathbb{R}$.

The existence of such a function is proven in Appendix B.

Let $\Lambda$ be some finite, rectangular array of sites. Let $d \mu_{I_{\beta}}(\phi)$ be a measure with the following properties:

i)

$$
\int e^{i \phi(f)} d \mu_{I_{\beta}}(\phi)=0
$$

for all real functions $f$ on $\Lambda$ with $\sum_{j} f(j) \neq 0$.

ii) Let $\sigma$ be a complex function on $\Lambda$ with $\|\operatorname{Im} \sigma\|_{\infty} \leqq \beta / 2$. Then

$$
\frac{d \mu_{I_{\beta}}(\phi+\sigma)}{d \mu_{I_{\beta}}(\phi)}=\mathscr{J}_{\beta}(\nabla(\phi+\sigma)),
$$

where

$$
\mathscr{I}_{\beta}(\nabla \phi, \nabla \sigma)=\prod_{\substack{|i-j|=1 \\ i \in A}} \frac{I_{\beta}(\phi(i)-\phi(j)+\sigma(i)-\sigma(j))}{I_{\beta}(\phi(i)-\phi(j))} .
$$

Remark. A measure $d \mu_{I_{\beta}}(\phi)$ with properties i) and ii) can be obtained from the family of measures

$$
d \mu_{I_{\beta}, \Omega}(\phi)=N_{\Omega}^{-1} \prod_{\substack{|i-j|=1 \\ i \in \Omega}} I_{\beta}(\phi(i)-\phi(j)) \prod_{j \in \Omega} d \phi(j),
$$

with $\phi(j)=0$, for $j \notin \Omega$, in the limit $\Omega \rtimes \mathbb{Z}^{2}$, by means of a weak compactness argument. In this case we shall speak of free b.c. at $\partial \Lambda$. Alternatively, we may 
choose $d \mu_{I_{\beta}}(\phi)$ to be given by the measure $d \mu_{I_{\beta}, \Lambda}(\phi)$, but with periodic b.c. imposed at $\partial \Lambda$. The Gibbs state in the region $\Lambda$ (with free or periodic b.c. at $\partial \Lambda$ ) of the model dual to the plane rotator model is given by the measure

$$
Z_{\Lambda}^{-1} \prod_{j \in A}\left\{\sum_{q \in \mathbb{Z}} e^{i 2 \pi q \phi(j)}\right\} d \mu_{I_{\beta}}(\phi) .
$$

We recall that for small $\beta$ the Gibbs state of the plane rotator model in the thermodynamic limit is independent of b.c. In order to establish the existence of a Kosterlitz-Thouless transition we are therefore free to impose some b.c. at $\partial \Lambda$ which are technically convenient for large $\beta$. The Fourier transformation of the measure (6.5) with free or periodic b.c. at $\partial \Lambda$ is a Gibbs state of the rotator model with b.c. that are technically convenient for large $\beta$. More natural would be Dirichlet (i.e. zero) b.c. at $\partial \Lambda$ which our methods permit, in principle, to analyze, too (see Appendix D).

Next, we recall from Sect. 1.4 that, for $x=(n, 0)$,

$$
\hat{D}_{0 x}^{\xi}=\prod_{m=1}^{n} \frac{I_{\beta}\left(\left(\partial_{2} \phi\right)(j)+\xi\right)}{I_{\beta}\left(\left(\partial_{2} \phi\right)(j)\right)}=\mathscr{J}_{\beta}(\nabla \phi, \psi)
$$

where $\psi=\left(\psi_{1}, \psi_{2}\right)$, with $\psi_{1} \equiv 0$ and

$$
\psi_{2}(j)= \begin{cases}\xi, & \text { for } j=(i, 0), 1 \leqq i \leqq n \\ 0, & \text { otherwise }\end{cases}
$$

By (6.1) and (6.6)

$$
\left\langle e^{i \xi\left(\theta_{0}-\theta_{x}\right)}\right\rangle_{\Lambda}(\beta)=Z_{\Lambda}^{-1} \int \prod_{j \in \Lambda}\left\{\sum_{q=-\infty}^{\infty} e^{i 2 \pi q \phi(j)}\right\} \mathscr{J}_{\beta}(\nabla \phi, \psi) d \mu_{I_{\beta}}(\phi) .
$$

Clearly,

$$
\sum_{q=-\infty}^{\infty} e^{i 2 \pi q \phi(j)}=1+2 \sum_{q=1}^{\infty} \cos (2 \pi q \phi(j))
$$

Let $\left\{\zeta_{q}\right\}_{q=1}^{\infty}$ be a sequence of positive numbers such that $\sum_{q=1}^{\infty} \zeta_{q}=1$. We set $z(2 \pi q)$ $=2 \zeta_{q}^{-1}$. Then

$$
1+2 \sum_{q=1}^{\infty} \cos (2 \pi q \phi)=\sum_{q=1}^{\infty} \zeta_{q}(1+z(2 \pi q) \cos (2 \pi q \phi)) .
$$

Hence, by Theorem 5.2 and (5.40), Sect. 5.2

$$
\prod_{j \in \Lambda}\left[1+2 \sum_{q=1}^{\infty} \cos (2 \pi q \phi(j))\right]=\sum_{\mathscr{N} \in \mathscr{F}_{\mathcal{A}}} c_{\mathscr{N}} \prod_{\varrho \in \mathscr{N}}(1+K(\varrho) \cos \phi(\varrho)), c_{\mathscr{N}}>0,
$$

where each $\mathscr{N} \in \mathscr{F}_{i}$ satisfies properties a)-c) stated in Theorem 2.1 and

$$
K(\varrho) \leqq\left\{\prod_{j \in \text { supp } \varrho} z(|\varrho(j)|)\right\} e^{S(\varrho)}
$$

with $S(\varrho)$ as in Theorems 2.1 and 5.2 ; finally $\varrho(j) \in 2 \pi \mathbb{Z}$, for all $j$. 
Thus

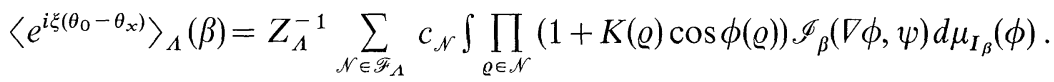

By property a), Theorem 2.1, and (6.3) we may assume that each $\varrho \in \mathscr{N}$ is neutral, i.e. $\sum \varrho(j)=0$, for all $\mathscr{N} \in \mathscr{F}_{\Lambda}$.

As in the Villain model [see (5.53), Sect. 5.2] we shall make the change of variables

where

$$
\left.\begin{array}{c}
\phi(j) \rightarrow \phi(j)+\sigma(j), \\
\sigma(j)=\xi\left(C^{\prime} * \partial_{2} f^{x}\right)(j),
\end{array}\right\}
$$

$f^{x}$ is the function defined in (5.49), and $C^{\prime}$ is the Green's function of $-\left(\partial_{1}^{2}+\partial_{2}^{2}\right)$.

By Eq. (5.55),

$$
\xi^{-1}\left(\partial_{1} \sigma\right)(j)=\partial_{2}\left(C^{\prime}(j)-C^{\prime}(j-x)\right)
$$

and

$$
\begin{aligned}
\xi^{-1}\left(\partial_{2} \sigma\right)(j) & =\left(\partial_{1}^{2}+\partial_{2}^{2}\right)\left(C^{\prime} * f^{x}\right)(j)-\partial_{1} C^{\prime} * \partial_{1} f^{x}(j) \\
& =-f^{x}(j)-\partial_{1}\left(C^{\prime}(j)-C^{\prime}(j-x)\right) .
\end{aligned}
$$

We now define

and

$$
\left.\begin{array}{rl}
\theta_{1}(j) & =\xi \partial_{2}\left(C^{\prime}(j)-C^{\prime}(j-x)\right), \\
\theta_{2}(j) & =-\xi \partial_{1}\left(C^{\prime}(j)-C^{\prime}(j-x)\right), \\
\theta & =\left(\theta_{1}, \theta_{2}\right) .
\end{array}\right\}
$$

In each term on the right side of (6.9) we now make the change of variables (6.10). By (6.4), (6.6), and (6.12)

$$
\begin{aligned}
\mathscr{I}_{\beta}(\nabla \phi, \psi) d \mu_{I_{\beta}}(\phi) & \rightarrow \mathscr{J}_{\beta}(\nabla(\phi+\sigma), \psi) \mathscr{I}_{\beta}(\nabla \phi, \nabla \sigma) d \mu_{I_{\beta}}(\phi) \\
& =\mathscr{J}_{\beta}(\nabla \phi, \theta) d \mu_{I_{\beta}}(\phi),
\end{aligned}
$$

since the term $-\xi f^{x}$ in $\partial_{2} \sigma$ cancels $\psi$; see (6.11)-(6.13) and (6.6).

Thus

$$
\left\langle e^{i \xi\left(\theta_{0}-\theta_{x}\right)}\right\rangle_{\Lambda}(\beta)=Z_{\Lambda}^{-1} \sum_{\mathscr{N} \in \mathscr{F}_{A}} c_{\mathscr{N}} Z_{\mathscr{N}^{\prime}}^{\prime}(\sigma),
$$

where

$$
Z_{\mathcal{N}}^{\prime}(\sigma)=\int \prod_{\varrho \in \mathscr{N}}[1+K(\varrho) \cos (\phi(\varrho)+\sigma(\varrho))] \mathscr{J}_{\beta}(\nabla \phi, \theta) d \mu_{I_{\beta}}(\phi) .
$$

Our main task is now to prove a lower bound on $Z_{\mathcal{N}}^{\prime}(\sigma)$ : For all $\beta>\beta_{0}$, for some finite $\beta_{0}$

$$
Z_{\mathscr{N}}^{\prime}(\sigma) \geqq \exp \left[-\left(1 / 2 \beta^{\prime}\right)\langle\theta, \theta\rangle\right] Z_{\mathscr{N}}^{\prime}(0),
$$

for some positive $\beta^{\prime}(\beta)$ which tends to $+\infty$ when $\beta \rightarrow \infty$. In order to prove (6.17) we must first renormalize the factors $1+K(\varrho) \cos (\phi(\varrho)+\sigma(\varrho))$ under the integral on the right side of (6.16), using the techniques of Sect. 4. The result is the following variant of Theorem 4.1. 
Theorem 6.1. Let $I_{\beta}(\phi)$ be a function satisfying properties (a) through (d), let $d \mu_{I_{\beta}}$ be a measure satisfying (6.3) and (6.4). Finally let $\mathscr{N}$ be an ensemble of charge densities with the properties described in Theorems 2.1 and 5.2. Then

$$
\begin{aligned}
Z_{\mathcal{N}}^{\prime}(\sigma)= & \int \prod_{\varrho \in \mathcal{N}}\left[1+z(\beta, \varrho) F_{1, \beta}(\varrho ; \nabla \phi+\theta) \sin (\phi(\varrho)+\sigma(\varrho))\right. \\
& \left.+z(\beta, \varrho) F_{2, \beta}(\varrho ; \nabla \phi+\theta) \cos (\phi(\varrho)+\sigma(\varrho))\right] \mathscr{J}_{\beta}(\nabla \phi+\theta) d \mu_{I_{\beta}}(\phi),
\end{aligned}
$$

where

$$
\begin{gathered}
z(\beta, \varrho)=K(\varrho) \exp \left[-L(\beta) E_{\mathrm{loc}}(\varrho)\right] \\
E_{\mathrm{loc}}(\varrho) \geqq C\|\varrho\|_{1}+D \sum_{k=1}^{\infty}\left|\mathscr{S}_{k}^{\prime}(\varrho)\right|
\end{gathered}
$$

with $\mathscr{S}_{k}^{\prime}(\varrho)$ as in (3.3), Sect. 3 (see also (4.58), Sect. 4). Here $L(\beta)$ is some function of $\beta$ which tends to $\infty$, as $\beta \rightarrow \infty$ (one may choose $L(\beta) \geqq$ const $\ln \beta$ ). Moreover, the functionals $F_{i, \beta}(\varrho ; \cdot)$ have the following properties:

(1) $F_{i}(\varrho ; \nabla \phi)$ depends only on

$$
\left\{\partial_{\alpha} \phi(j): \alpha=1,2, j \in D(\varrho)\right\}
$$

where $D(\varrho)$ is the disc defined at the beginning of Sect. 4.3, i.e. $D(\varrho) \supset$ supp $\varrho$, $\operatorname{dist}(\operatorname{supp} \varrho, \partial D(\varrho))=d(\varrho)$.

(2) $F_{1}$ is odd, $F_{2}$ is even in the variables $\nabla \phi(j)$.

(3) $\left\|F_{i}(\varrho ; \cdot)\right\|_{\infty} \leqq 1$, for real $\phi$.

(4) For $i=1,2$, for arbitrary real $\phi$ and for $m=1,2$,

$$
\left|\frac{\partial^{m}}{\partial \lambda^{m}} F_{i}(\varrho ; \nabla \phi+\lambda \theta)\right| \leqq \operatorname{const} d(\varrho)^{2 m} \max _{j \in D(\varrho)}|\theta(j)|^{m},
$$

provided $\beta$ is sufficiently large. Here $|\theta| \equiv \sqrt{\theta_{1}^{2}+\theta_{2}^{2}}$.

Proof. The structure of the proof of Theorem 6.1 is identical to the one of Theorem 4.1 which was given in Sect. 4.4. The renormalization transformation is carried out by induction in the size of $\varrho$ and in the scale size, $2^{\ell}, \ell=0,1,2, \ldots$, of the charged constituents of $\varrho$. On scale $2^{0}$ the renormalization is obtained by applying Lemma 4.3 and (4.22)-(4.24). The renormalization on scales $2^{\ell}, \ell \geqq 1$, is carried out with the help of Lemma 4.4, Sect. 4.3. In order to verify properties $(2)$ and (4) of $F_{i}(\varrho ; \cdot)$ we shall need the explicit expression for the renormalization $\mathrm{RC}_{1}$ of the functional $C_{1}(\phi, \varrho)$ given in Eq. (4.40). Equations (4.15), (4.22), (4.24), Sect. 4.2, and Eqs. (4.39), (4.40), Sect. 4.3, show that $F_{1}$ and $F_{2}$ have the following general form:

$$
\begin{aligned}
i^{n-2} 2 F_{n}(\varrho ; \nabla \phi)= & \prod_{\substack{j \in D(\varrho) \\
\alpha=1,2}} i_{\beta}\left(\partial_{\alpha} a(j) ; \partial_{\alpha} \phi(j)\right) \\
& +(-1)^{n} \prod_{\substack{j \in D(\varrho) \\
\alpha=1,2}} i_{\beta}\left(-\partial_{\alpha} a(j) ; \partial_{\alpha} \phi(j)\right),
\end{aligned}
$$

where $n=1,2$ and

$$
i_{\beta}(a ; \phi)=I_{\beta}(\phi+i a) I_{\beta}(\phi)^{-1} e^{-g(a) / \beta}
$$


see (4.12), Sect. 4.2, and property (c) of $I_{\beta}$. The "translation function" $a$ is of the form

$$
a(j)=\sum_{\ell, m} a_{\ell, m}(j),
$$

where $\ell$ labels the scale size and $m$ labels elements of $\mathscr{S}_{\ell}^{\prime}(\varrho)$. [The functions $a_{0, m}$ come from applying Lemma 4.3, and $a_{\ell, m}$ is the translation function introduced in the renormalization of the $m^{\text {th }}$ square in $\mathscr{S}_{\ell}^{\prime}(\varrho)$, using Lemma 4.4.] Clearly $a_{\ell, m}$ depends on $\varrho, \operatorname{supp} a_{\ell, m} \subseteq D(\varrho)$, for all $\ell, m$, and

$$
\operatorname{supp} \nabla a_{\ell, m} \cap \operatorname{supp} \nabla a_{\ell^{\prime}, m^{\prime}}=\emptyset
$$

unless $(\ell, m)=\left(\ell^{\prime}, m^{\prime}\right)$.

Properties (1)-(3) stated in Theorem 6.1 follow from these properties of $a$ and from Eq. (6.18) by taking into account that

$$
\left\|i_{\beta}(a ; \cdot)\right\|_{\infty} \leqq 1
$$

[see (4.12), Sect. 4.2].

Our lower bound on $E_{\text {loc }}(\varrho)$ and $L(\beta)$ is proven as follows: The renormalization on scale $2^{0}$ contributes an amount const $\ln \beta\|\varrho\|_{1}$ to $L(\beta) E_{\text {loc }}(\varrho)$. This follows from (4.16), (4.20), (4.21), and (4.24) by choosing $a_{0, m}(j)=$ const $\operatorname{sign} \varrho(m)(\ln \beta) \delta_{j m}$, for some sufficiently small constant. By property (c) of the function $I_{\beta}$, this is an allowed choice for $a_{0}$. The term $D \sum_{k=1}^{\infty}\left|\mathscr{P}_{k}^{\prime}(\varrho)\right|$ in $E_{\text {loc }}(\varrho)$ is obtained by inductive renormalization, as in Theorem 4.1, each induction step being carried out with the help of Lemma 4.4. By (6.20) and property (c) of $I_{\beta}$ the translation functions $a_{\ell, m}$ can be chosen as in (4.37), Sect. 4.3, with $\gamma=\operatorname{const} \ln \beta$, for some sufficiently small constant. We set $R=\sqrt{2}$ in (4.34) and (4.35), as in the proof of Theorem 4.1. For $\ell \geqq O(\ln \beta)$ we can in fact choose $\gamma=$ const $\beta$ [see (4.34)]. It follows now from (4.34) that $L(\beta) \geqq$ const $\ln \beta$. [By improving the inequality in property (c) of $I_{\beta}$ one might be able to show that $L(\beta)$ grows like a small power of $\beta$.] This completes the proof of the lower bounds on $E_{\text {loc }}(\varrho)$ and $L(\beta)$. The bound on $z(\beta, \varrho)$ follows from the bound on $L(\beta) E_{\text {loc }}(\varrho)$ and Theorem 5.2.

Thus, we are left with proving property $(4)$ of $F_{i}(\varrho ; \cdot)$. We make use of the explicit expressions for $F_{1}$ and $F_{2}$ given in Eq. (6.18) and of property (d) of $I_{\beta}$. We first note that for each $j \in D(\varrho)$ and $\kappa=1,2$,

$$
\begin{aligned}
& \frac{\partial}{\partial \lambda} i_{\beta}\left(\partial_{\kappa} a(j) ; \partial_{\kappa} \phi(j)+\lambda \theta_{\kappa}(j)\right)=e^{-g\left(\partial_{\kappa} a(j)\right) / \beta} \frac{\partial}{\partial \lambda}\left(I_{\beta}\left(r(\lambda)+i \partial_{\kappa} a(j)\right) / I_{\beta}(r(\lambda))\right) \\
& \quad=\theta_{\kappa}(j)\left[\left(\log I_{\beta}\right)^{\prime}\left(r(\lambda)+i \partial_{\kappa} a(j)\right)-\left(\log I_{\beta}\right)^{\prime}(r(\lambda))\right] i_{\beta}\left(\partial_{\kappa} a(j) ; \partial_{\kappa} \phi(j)+\lambda \theta_{\kappa}(j)\right),
\end{aligned}
$$

where $r(\lambda) \equiv \partial_{\kappa} \phi(j)+\lambda \theta_{\kappa}(j)$ which is real. Now from properties (c) and (d) of $I_{\beta}$ we see that the absolute value of the expression in (6.22) is bounded by

$$
2 C \beta^{-1}\left[\exp 2 \pi\left|\partial_{\kappa} a(j)\right|\right]\left|\theta_{\kappa}(j)\right| .
$$

Similarly the second derivative of $i_{\beta}\left(\partial_{\kappa} a(j) ; \partial_{\kappa} \phi(j)+\lambda_{\kappa} \theta_{\kappa}(j)\right)$ in $\lambda$ is bounded in absolute value by

$$
6 C \beta^{-1}\left[\exp 2 \pi\left|\partial_{\kappa} a(j)\right|\right] \cdot\left|\theta_{\kappa}(j)\right|^{2} .
$$


By (6.18), (6.22), and (6.23) the first derivative of $F_{1}$ or $F_{2}$ in $\lambda$ produces no more than const $d(\varrho)^{2}$ terms, each term bounded by

$$
C \beta^{-1} \max _{j \in D(\varrho)}\left(\left[\exp 2 \pi\left|\partial_{\kappa} a(j)\right|\right]\left|\theta_{\kappa}(j)\right|\right) .
$$

The bound on the second derivatives is obtained by similar arguments.

The proof of (4) is completed by requiring that

$$
\beta^{-1} \exp 2 \pi\left|\partial_{\kappa} a(j)\right| \leqq \text { const. }
$$

Thanks to property $(6.20),(6.25)$ holds if

$$
\beta^{-1} \exp 2 \pi\left|\partial_{\kappa} a_{\ell, m}(j)\right| \leqq \mathrm{const},
$$

for all $\ell$ and $m$, i.e.

$$
\left|a_{\ell, m}(j)\right| \leqq c \ln \beta .
$$

This bound is fulfilled by the functions $a_{\ell, m}$ used in the proof of the lower bound on $L(\beta) E_{\text {loc }}(\varrho)$.

Remark. As in Theorem 5.3, Sect. 5.2, one convinces oneself that Theorem 6.1 yields the following bound on $z(\beta, \varrho)$ :

$$
0<z(\beta, \varrho)<\exp \left[-\left(c^{\prime \prime} L(\beta)-b^{\prime \prime}\right)\|\varrho\|_{1}\right] \cdot \exp (-(c L(\beta)-b) A(\varrho)],
$$

for some positive constants $c^{\prime \prime}, c$ and finite $b^{\prime \prime}$ and $b$, provided $L(\beta)$ is large enough, i.e. for sufficiently large $\beta$.

We now complete our proof of the basic lower bound on $\left\langle e^{i \xi\left(\theta_{0}-\theta_{x}\right)}\right\rangle(\beta), 0 \neq$ $\xi \in \mathbb{Z}$, stated in (6.1). Our arguments are very similar to the ones used in the proof of Theorem 5.4, Sect. 5.2, concerning the Villain model. We recall identity (6.15), i.e.

$$
\left\langle e^{i \xi\left(\theta_{0}-\theta_{x}\right)}\right\rangle_{\Lambda}(\beta)=Z_{\Lambda}^{-1} \sum_{\mathcal{N} \in \mathscr{F}_{A}} c_{\mathscr{N}} Z_{\mathscr{N}}^{\prime}(\sigma),
$$

where $Z_{\mathcal{N}}^{\prime}(\sigma)$ is given in Theorem 6.1. In order to establish (6.1) it suffices to show that

$$
Z_{\mathscr{N}}^{\prime}(\sigma) \geqq \text { const } \exp \left[-\left(2 \beta^{\prime}\right)^{-1}\|\theta\|_{2}^{2}\right] Z_{\mathscr{N}},
$$

for some positive $\beta^{\prime}$, provided $\beta$ is large enough [see (6.17)]. For, since

$$
\begin{aligned}
\|\theta\|_{2}^{2} & =\sum_{j}\left\{\left|\theta_{1}(j)\right|^{2}+\left|\theta_{2}(j)\right|^{2}\right\} \\
& =2 \xi^{2}\{C(0)-C(x)\} \\
& \approx \xi^{2}(\pi)^{-1} \ln |x|, \quad \text { for large }|x|,
\end{aligned}
$$

and

$$
Z_{\Lambda}^{-1} \sum_{\mathscr{N} \in \mathscr{F}_{\Lambda}} c_{\mathscr{N}} Z_{\mathscr{N}}=1
$$

inequality (6.27) yields (6.1).

We prove (6.27) by using Theorem 6.1 and Jensen's inequality, as in the proof of Theorem 5.1, (5.12)-(5.21). 
First we consider the factor $\mathscr{J}_{\beta}(\nabla \phi+\theta)$ under the integral expressing $Z^{\prime}(\sigma)$. By property $(\mathrm{d})$ of $I_{\beta}(\phi)$ and Taylor's theorem with remainder,

$$
I_{\beta}\left(\partial_{\kappa} \phi(j)+\theta_{\kappa}(j)\right) I_{\beta}\left(\partial_{\kappa} \phi(j)\right)^{-1} \geqq \exp \left[\left(\frac{I_{\beta}^{\prime}}{I_{\beta}}\right)\left(\partial_{\kappa} \phi(j)\right) \theta_{\kappa}(j)-C \beta^{-1}\left(\theta_{\kappa}(j)\right)^{2}\right],
$$

where $I_{\beta}^{\prime}(\phi)=\left(\frac{\partial}{\partial \phi} I_{\beta}\right)(\phi)$. By property (b) of $I_{\beta}, I_{\beta}^{\prime} I_{\beta}^{-1}$ is an odd function of $\phi$. Thus, by (6.4), (6.13), and (6.14)

$$
\mathscr{I}_{\beta}(\nabla \phi+\theta) \geqq \exp \left[H_{0}(\phi)-C \beta^{-1}\|\theta\|_{2}^{2}\right],
$$

where $H_{0}$ is an odd function of $\phi$. The factor $\exp \left[-C \beta^{-1}\|\theta\|_{2}^{2}\right]$ is the analogue of the spin wave contribution, $\exp G(x)$, in the Villain model; see (5.54), (5.56). We define

$$
\begin{aligned}
F_{\theta, \beta}(\varrho ; \nabla \phi)= & F_{1, \beta}(\varrho ; \nabla \phi+\theta) \sin \left(\phi(\varrho)+[\sigma(\varrho)]_{2 \pi}\right) \\
& +F_{2, \beta}(\varrho ; \nabla \phi+\theta) \cos \left(\phi(\varrho)+[\sigma(\varrho)]_{2 \pi}\right) .
\end{aligned}
$$

Thanks to the periodicity of $\sin$ and cos we now have

$$
Z^{\prime}(\sigma) \geqq \exp \left[-C \beta^{-1}\|\theta\|_{2}^{2}\right] \int \prod_{\varrho \in \mathcal{N}}\left[1+z(\beta, \varrho) F_{\theta, \beta}(\varrho ; \nabla \phi)\right] e^{H_{0}(\phi)} d \mu_{I_{\beta}}(\phi),
$$

and we emphasize that the renormalized activites, $z(\beta, \varrho)$, are independent of $\sigma$ and $\theta$. As in (5.17), Sect. 5.1, we write

$$
1+z(\beta, \varrho) F_{\theta, \beta}(\varrho ; \nabla \phi)=\exp \ln [1+X+Y],
$$

where

$$
\begin{gathered}
X=z(\beta, \varrho) F_{\theta=0, \beta}(\varrho ; \nabla \phi), \\
Y=z(\beta, \varrho)\left[F_{\theta, \beta}(\varrho ; \nabla \phi)-F_{0, \beta}(\varrho ; \nabla \phi)\right] .
\end{gathered}
$$

By (6.26) we may choose $\beta$ so large that $z(\beta, \varrho)<1 / 8$. Then, by Theorem $6.1,(3)$

and

$$
(1+X)^{-1} \leqq \frac{4}{3}
$$

$$
(1+X+t Y)^{-1} \leqq 2, \text { for all } t \in(0,1)
$$

Taylor's theorem with remainder then gives

$$
1+z(\beta, \varrho) F_{\theta, \beta}(\varrho ; \nabla \phi)=(1+X) \exp \left[\frac{Y}{1+X}-\frac{1}{2}\left(\frac{Y}{1+X+t Y}\right)^{2}\right]
$$

for some $t \in(0,1)$. Abbreviating

$$
F_{\lambda \theta, \beta}(\varrho ; \nabla \phi)-F_{0, \beta}(\varrho, \nabla \phi), \quad 0 \leqq \lambda \leqq 1,
$$

by $F_{\lambda}(\phi)$ we obtain from Taylor's theorem

$$
Y=z(\beta, \varrho)\left[\frac{\partial}{\partial \lambda} F_{\lambda}(\phi)\right]_{\lambda=0}+\frac{z(\beta, \varrho)}{2}\left[\frac{\partial^{2}}{\partial \lambda^{2}} F_{\lambda}(\phi)\right]_{\lambda=\lambda_{0}},
$$

for some $\lambda_{0} \in(0,1)$. 
We now define

$$
H_{1, \varrho}(\phi)=z(\beta, \varrho)(1+X)^{-1}\left[\frac{\partial}{\partial \lambda} F_{\lambda}(\phi)\right]_{\lambda=0} .
$$

By Theorem 6.1, (2) and the definition of $X$ and $F_{\lambda}, H_{1, \varrho}$ is an odd function of $\phi$. Next, let

$$
H_{2, \varrho}(\phi)=\frac{z(\beta, \varrho)}{2}(1+X)^{-1}\left[\frac{\partial^{2}}{\partial \lambda^{2}} F_{\lambda}(\phi)\right]_{\lambda=\lambda_{0}}-\frac{1}{2}(Y / 1+X+t Y)^{2}
$$

and

$$
\left|H_{2, \varrho}\right|=\sup _{\phi, \lambda_{0}, t}\left|H_{2, \varrho}(\phi)\right| .
$$

Then, by (6.31) and (6.32)-(6.37),

$$
\begin{aligned}
Z_{\mathscr{N}}^{\prime}(\sigma) \geqq & \exp \left[-C \beta^{-1}\|\theta\|_{2}^{2}\right] \\
& \cdot \int \prod_{\varrho \in \mathscr{N}}\left\{\left[1+z(\beta, \varrho) F_{0, \beta}(\varrho ; \nabla \phi)\right] e^{H_{1, \varrho}(\phi)} e^{-\left|H_{2, \varrho}\right|}\right\} e^{H_{0}(\phi)} d \mu_{I_{\beta}}(\phi) .
\end{aligned}
$$

Since, by Theorem $6.1,(2)$, for large $\beta$

$$
\prod_{\varrho \in \mathscr{N}}\left[1+z(\beta, \varrho) F_{0, \beta}(\varrho ; \nabla \phi)\right] d \mu_{I_{\beta}}(\phi)
$$

is positive and even in $\phi$, whereas $H_{0}(\phi)$ and $\sum_{\varrho \in \mathcal{N}} H_{1, \varrho}(\phi)$ are real-valued, odd functions of $\phi$, Jensen's inequality yields

$$
Z_{\mathscr{N}}^{\prime}(\sigma) \geqq \exp \left[-C \beta^{-1}\|\theta\|_{2}^{2}\right]\left(\prod_{\varrho \in \mathscr{N}} e^{-\left|H_{2, e}\right|}\right) Z_{\mathscr{N}},
$$

where

$$
Z_{\mathcal{N}}=\int \prod_{\varrho \in \mathscr{N}}\left[1+z(\beta, \varrho) F_{0, \beta}(\varrho ; \nabla \phi)\right] d \mu_{I_{\beta}}(\phi) .
$$

We proceed to proving a bound on $\left|H_{2, \varrho}\right|$. We use Theorem 6.1 , (4) and the following equations for $[\sigma(\varrho)]_{2 \pi}$,

$$
[\sigma(\varrho)]_{2 \pi}=\left[\sum_{\mu} 2 \pi\left(\sigma\left(p_{\mu}\right)-\sigma\left(n_{\mu}\right)\right)\right]_{2 \pi},
$$

and there are no more than $1 / 2\left\|(2 \pi)^{-1} \varrho\right\|_{1}$ terms on the right side of $(6.40)$. Furthermore

$$
\left[2 \pi\left(\sigma\left(p_{\mu}\right)-\sigma\left(n_{\mu}\right)\right)\right]_{2 \pi}=\left[\sum_{j} 2 \pi\left(\varepsilon_{1}(j) \theta_{1}(j)+\varepsilon_{2}\left(j^{\prime}\right) \theta_{2}\left(j^{\prime}\right)\right)\right]_{2 \pi},
$$

where $j^{\prime}$ is some nearest neighbor of $j$, and $\varepsilon_{\kappa}(j)=0, \pm 1$, $\operatorname{supp} \varepsilon_{\kappa} \subseteq D(\varrho)$, for $\kappa=1,2$, and

$$
\sum_{j}\left\{\left|\varepsilon_{1}(j)\right|+\left|\varepsilon_{2}(j)\right|\right\} \leqq 2 d(\varrho) .
$$

The proof of these equations is identical to the one of Eqs. (5.59), (5.60), Sect. 5.2 [replacing $C$ by $C^{\prime}, \sigma$ by $2 \pi \sigma$ and taking into account the definition (6.13) of $\theta$ ]. 
If we recall the definition (6.37) of $H_{2, \varrho}$ and (6.33) and use Theorem 6.1, (4) and (6.40)-(6.42) we conclude

$$
\left|H_{2, \varrho}\right| \leqq \operatorname{const} z(\beta, \varrho) d(\varrho)^{4}\left\|(2 \pi)^{-1} \varrho\right\|_{1}^{2} \max _{j \in D(\varrho)}|\theta(j)|^{2},
$$

provided $\beta$ is so large that

$$
(1+X)^{-1} \leqq \frac{4}{3}, \quad(1+X+t Y)^{-1} \leqq 2
$$

[see (6.34)]. It now suffices to note that

$$
\max _{j \in D(\varrho)}|\theta(j)|^{2} \leqq \operatorname{const} d(\varrho)^{2} \max _{j \in \operatorname{supp} \varrho}|\theta(j)|^{2} .
$$

It now follows from the upper bound (6.26) on $z(\beta, \varrho)$ that

$$
\left|H_{2, \varrho}\right| \leqq c(\beta) \max _{j \in \operatorname{supp} \varrho}|\theta(j)|^{2},
$$

where $c(\beta)$ tends to 0 , as $\beta \rightarrow \infty$ (like some power of $1 / \beta$ ). Since supp $\varrho \cap \operatorname{supp} \varrho^{\prime}=\emptyset$, for two distinct charge densities $\varrho$ and $\varrho^{\prime}$ in $\mathscr{N}$

$$
\sum_{\varrho \in \mathcal{N}} \max _{j \in \operatorname{supp} \varrho}|\theta(j)|^{2} \leqq\|\theta\|_{2}^{2},
$$

hence

$$
Z_{\mathscr{N}}^{\prime}(\sigma) \geqq \exp \left[-\left(C \beta^{-1}+c(\beta)\right)\|\theta\|_{2}^{2}\right] Z_{\mathscr{N}},
$$

provided $\beta$ is sufficiently large.

This completes the proof of inequality (6.27), with $2 \beta^{\prime}=\left(C \beta^{-1}+c(\beta)\right)^{-1}$. Since the basic lower bound (6.1) on $\left\langle e^{i \xi\left(\theta_{0}-\theta_{x}\right)}\right\rangle_{\Lambda}(\beta)$ follows from (6.27) - as explained the proof of existence of a Kosterlitz-Thouless transition in the rotator model is complete.

\section{Massless Phase in Two-Dimensional $\mathbb{Z}_{n}$ Models and Roughening Transition in the Solid-on-Solid Model}

In this section we first establish the existence of a massless, intermediate phase in the two-dimensional $\mathbb{Z}_{n}$-models. (We prove Theorem E, Sect. 1.4.) We then exhibit a Kosterlitz-Thouless, or roughening transition for the solid-on-solid model, defined in (1.21) (see Theorem A, Sect. 1.3).

\subsection{The $\mathbb{Z}_{n}-$ Models}

Let $d \mu_{\beta}(\theta)$ denote the unique Gibbs measure of the two-dimensional rotator model in the thermodynamic limit. The Gibbs measure, $d \mu_{\beta, \Lambda}^{(n)}(\theta)$ of the $\mathbb{Z}_{n}$-model in a finite domain $\Lambda \subset \mathbb{Z}^{2}$ with "free" boundary conditions is given in terms of $d \mu_{\beta}(\theta)$ by the equation

$$
d \mu_{\beta, \Lambda}^{(n)}(\theta)=\left(Z_{\Lambda}^{(n)}\right)^{-1} \prod_{j \in \Lambda}\left(1+2 \sum_{q=1}^{\infty} \cos \left(q n \theta_{j}\right)\right) d \mu_{\beta}(\theta)
$$


where $Z^{(n)}$ is the usual partition function, and

$$
\left(1+2 \sum_{q=1}^{\infty} \cos (q n \theta)\right) d \theta=\left(\sum_{k=0}^{n-1} \delta(n \theta-2 \pi k)\right) d \theta
$$

is the Dirac measure with support on $\{\theta: \theta=2 \pi k / n, k=0,1, \ldots, n-1\}$.

Let $\langle\cdot\rangle_{A}^{(n)}(\beta)$ denote the expectation with respect to $d \mu_{\beta, \Lambda}^{(n)}(\theta)$; see Sect. 1.4, (1.38). The thermodynamic limit, $\langle\cdot\rangle^{(n)}(\beta)$, (as $\Lambda \rtimes \mathbb{Z}^{2}$ ) exists by Ginibre's inequalities [11].

Our main result for the $\mathbb{Z}_{n}$-models is

Theorem 7.1. For all $\xi=1, \ldots, n-1$,

(1) $\left\langle\mathrm{e}^{i \xi\left(\theta_{0}-\theta_{x}\right)}\right\rangle^{(n)}(\beta) \geqq\left\langle\mathrm{e}^{i \xi\left(\theta_{0}-\theta_{x}\right)}\right\rangle(\beta)$.

(2) Given $\beta$ and $q \in(0,1)$, ther exists some $n_{0}=n_{0}(\beta, q)<\infty$ such that for all $n \geqq n_{0}$ and all $\xi$ with $\min (\xi / n, 1-\xi / n) \geqq q$

$$
\left\langle e^{i \xi\left(\theta_{0}-\theta_{x}\right)}\right\rangle^{(n)}(\beta) \leqq \operatorname{const}(1+|x|)^{-\left[\xi^{2} / 2 \pi \beta^{\prime \prime}\right]},
$$

for some $\beta^{\prime \prime}=\beta^{\prime \prime}(\beta, n, q)<\infty$. Moreover, $n_{0}(\beta, q) \rightarrow \infty$ when $\beta \rightarrow \infty$ or $q \rightarrow 0$.

Remarks. 1. It suffices to prove Theorem 7.1 for $\left\langle e^{i \xi\left(\theta_{0}-\theta_{x}\right)}\right\rangle_{\Lambda}^{(n)}(\beta)$ for arbitrary, finite domains $\Lambda$. The states $\langle\cdot\rangle_{\Lambda}^{(n)}(\beta)$ are the limits of the states $\langle\cdot\rangle_{\Lambda}^{(n)}(\beta, h)$, defined in Sect. 1.4, as $h \rightarrow \infty$. By Ginibre's inequality, $\left\langle e^{i \xi\left(\theta_{0}-\theta_{x}\right)}\right\rangle_{A}^{(n)}(\beta, h)$ is monotone increasing in $\Lambda$ and $h$. This proves part (1) of Theorem 7.1 and shows that Theorem 7.1 holds for all $h, 0<h \leqq \infty$, assuming it holds for $h=\infty$.

2. Let $\beta_{c}$ be the critical temperature of the plane rotator model. For $\beta>\beta_{c}$

$$
\left\langle e^{i \xi\left(\theta_{0}-\theta_{x}\right)}\right\rangle(\beta) \geqq \operatorname{const}(1+|x|)^{-\left[\xi^{2} / 2 \pi \beta^{\prime}\right]},
$$

for some $\beta^{\prime}=\beta^{\prime}(\beta)(\rightarrow \infty$, as $\beta \rightarrow \infty)$, as shown in Sect. 6. Thus, for $\beta>\beta_{c}$,

$$
\left\langle e^{i \xi\left(\theta_{0}-\theta_{x}\right)}\right\rangle^{(n)}(\beta) \geqq \operatorname{const}(1+|x|)^{-\left[\xi^{2} / 2 \pi \beta^{\prime}\right]} .
$$

Furthermore, for $n \geqq n_{0}(\beta, q)$ and $\min (\xi / n, 1-\xi / n) \geqq q$,

$$
\left\langle e^{i \xi\left(\theta_{0}-\theta_{x}\right)}\right\rangle_{\beta}^{(n)} \leqq \operatorname{const}(1+|x|)^{-\left[\xi^{2} / 2 \pi \beta^{\prime \prime}\right]},
$$

for some finite $\beta^{\prime \prime}$. Let $n_{0}(\beta)=\min _{0 \leqq q \leqq 1} n_{0}(\beta, q)$. [We shall see that $n_{0}(\beta)$ $=n_{0}\left(\beta, n^{-1}[n / 2]\right)$.] Inequalities (7.3) and (7.4) thus prove that, for $\beta>\beta_{c}$ and $n \geqq n_{0}(\beta)$, the inverse correlation length (mass gap), $m(\beta, n)$, of the $\mathbb{Z}_{n}$-model vanishes, i.e. there is a massless, intermediate phase for $\beta \in\left[\beta_{c}, \bar{\beta}_{c}\right)$, where $\beta_{c} \leqq \beta_{c}$ and $\bar{\beta}_{c}=\bar{\beta}_{c}(n)\left(>\beta_{c}\right.$, for $n$ large enough) is such that $n_{0}\left(\bar{\beta}_{c}\right) \geqq n$. For $\beta>\bar{\beta}_{c}$, the $\mathbb{Z}_{n^{-}}$ symmetry of the model is spontaneously broken. That $\bar{\beta}_{c}$ is finite is seen by a standard Peierls argument.

3. By methods closely related to the ones used in Sect. 6 one can show that for each $\beta$ there exists some positive integer $n_{1}(\beta)$ such that for all $n \geqq n_{1}(\beta)$

$$
\left\langle D_{0 x}^{\xi}\right\rangle^{(n)}(\beta) \geqq \operatorname{const}(1+|x|)^{-\xi^{2} \beta^{\prime \prime \prime} / 2 \pi},
$$

for some finite $\beta^{\prime \prime \prime}=\beta^{\prime \prime \prime}(\beta, n)$. Moreover $n_{1}(\beta) \rightarrow \infty$, as $\beta \rightarrow \infty$. Here $D_{0 x}^{\xi}$ is the disorder operator of the $\mathbb{Z}_{n}$ model, defined as in (1.31). The proof of (7.5) is almost identical to the one of inequality (6.1), after replacing $\phi$ by $\theta, I_{\beta}(\phi)$ by $\exp \beta \cos (\theta)$ 
and $d \mu_{I_{\beta}}(\phi)$ by $d \mu_{\beta}(\theta)$ in the formulas of Sect. 6 . The necessary estimates will follow from our subsequent analysis.

4. Instead of studying the $\mathbb{Z}_{n}$ models which correspond to the rotator model, we could also study $\mathbb{Z}_{n}$ models obtained from the Villain model by constraining $\theta_{j}$ to $\mathbb{Z}_{n}$, for all $j$. These models are self-dual, and a partial result concerning the existence of a massless, intermediate phase for $n$ large enough has previously been obtained in [15]. Our methods permit us to establish Theorem 7.1 and (7.5) for these models, as well.

We now turn to the proof of Theorem 7.1, (2). The basic ideas are straightforward variants of the ones developed in Sect. 5.1, in the proof of (5.27), i.e. of Theorem B, Sect. 1.2, for the hard core Coulomb gas, and of the ones in Sect. 6.

First, we choose a sequence of positive numbers, $\zeta_{q}$, such that

$$
\sum_{q=1}^{\infty} \zeta_{q}=1 \text { and } 0<z(q) \equiv 2 \zeta_{q}^{-1} \leqq e^{\text {const } q} .
$$

As in Sect. 5.2, (5.39) we have

$$
\prod_{j \in \Lambda}\left(1+2 \sum_{q=1}^{\infty} \cos \left(q n \theta_{j}\right)\right)=\sum_{q_{\Lambda}} \zeta\left(q_{\Lambda}\right) \prod_{j \in \Lambda}\left(1+z\left(q_{j}\right) \cos \left(q_{j} n \theta_{j}\right)\right),
$$

with

$$
q_{\Lambda}=\left\{q_{j}\right\}_{j \in \Lambda}, \quad \zeta\left(q_{\Lambda}\right) \equiv \prod_{j \in \Lambda} \zeta_{q_{J}} .
$$

We now rename $n \theta_{j}=\phi(j), e^{i \xi\left(\theta_{0}-\theta_{x}\right)}=e^{i \xi^{\prime}(\phi(0)-\phi(x))}$, with $\xi^{\prime}=\xi / n$. Clearly

$$
\left\langle e^{i \xi\left(\theta_{0}-\theta_{x}\right)}\right\rangle_{\Lambda}^{(n)}(\beta)=\left\langle\cos \phi\left(\xi^{\prime} \varrho_{0}\right)\right\rangle_{\Lambda}^{(n)}(\beta),
$$

with $\varrho_{0}=\delta_{j 0}-\delta_{j x}$. In our new notations it is obvious that we may apply Theorem 2.3 , Sect. 2.5 , which yields the identity

$$
\begin{aligned}
& \cos \phi\left(\xi^{\prime} \varrho_{0}\right) \prod_{j \in A}\left[1+z\left(q_{j}\right) \cos \left(q_{j} \phi(j)\right)\right] \\
& \quad \leqq \sum_{\mathscr{N} \in \mathscr{F}_{a_{A}}\left(\varrho_{0}\right)} c_{\mathscr{N}}^{\prime}\left(\cos \phi\left(\xi^{\prime} \varrho_{0}\right)+K\left(\varrho^{\mathscr{N}}\right) \cos \phi\left(\xi^{\prime} \varrho_{0}+\varrho^{\mathscr{N}}\right)\right) \prod_{\varrho \in \mathscr{N}}(1+K(\varrho) \cos \phi(\varrho)),
\end{aligned}
$$

and, by summing over all configurations, $q_{\Lambda}$,

$$
\begin{aligned}
& \cos \phi\left(\xi^{\prime} \varrho_{0}\right) \prod_{j \in \Lambda}\left[1+2 \sum_{q=1}^{\infty} \cos (q \phi(j))\right] \\
& =\sum_{\mathscr{N} \in \mathscr{F} \Lambda\left(\varrho_{0}\right)} c_{\mathscr{N}}\left(\cos \phi\left(\xi^{\prime} \varrho_{0}\right)+K\left(\varrho^{\mathscr{N}}\right) \cos \phi\left(\xi^{\prime} \varrho_{0}+\varrho^{\mathscr{N}}\right)\right) \prod_{\varrho \in \mathcal{N}}(1+K(\varrho) \cos \phi(\varrho)),
\end{aligned}
$$

where each $\mathscr{N} \in \mathscr{F}_{\Lambda}\left(\varrho_{0}\right)$ corresponds to some configuration $q_{\Lambda}$ and some $\mathscr{N}^{\prime} \in \mathscr{F}_{q_{\Lambda}}\left(\varrho_{0}\right)$, and $c_{\mathcal{N}}=\zeta\left(q_{\Lambda}\right) c_{\mathcal{N}}^{\prime}$, [see also Sect. 5.2, (5.39) and Theorem 5.2].

The estimates on the entropies of multipoles, i.e., the bare activities $K(\varrho)$, are as in Theorem 2.1 and 2.3, except that the factor $z^{A_{0}(\varrho)}$ must be replaced by $\prod_{j \in \text { supp } \varrho} z(|\varrho(j)|)$.

The remainder of the proof of Theorem 7.1, (2) is very similar to the proof of the analogous result (5.27), Sect. 5.1, for the hard core Coulomb gas, but the 
renormalization transformation in the present model is somewhat more complicated. It is similar to the one used in Sect. 6, Theorem 6.1. Again, the feature of the model which permits us to apply the renormalization method of Sect. 4 is the analyticity of the underlying measure, here $d \mu_{\beta}(\theta)=d \mu_{\beta}(\phi / n)$, in $\phi$. We shall follow closely the notations of Sect. 6 . Let

$$
\tilde{I}_{\beta}(\phi)=\exp \beta \cos (\theta)=\exp \beta \cos (\phi / n) .
$$

This function has the following properties:

(b) $\tilde{I}_{\beta}(\phi)$ is even and positive on the interval $[-\pi n, \pi n]$.

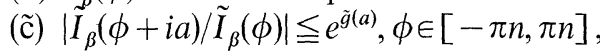
where

$$
0 \leqq \tilde{g}(a) \leqq \begin{cases}\operatorname{const} \beta\left(\frac{a}{n}\right)^{2}, & |a| \leqq n \\ \operatorname{const} \beta e^{|a| / n}, & n \leqq|a|<\infty .\end{cases}
$$

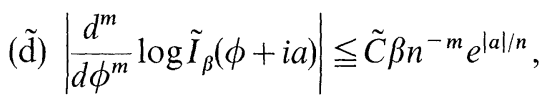

for $m=1,2$ and $|a|<\infty$, uniformly in $\phi \in[-\pi n, \pi n]$.

Properties $(\tilde{b})-(\tilde{d})$ follow by inspection from the formula

$$
\tilde{I}_{\beta}(\phi+i a)=\tilde{I}_{\beta}(\phi) \exp \{\beta \cos (\phi / n)(\cosh (a / n)-1)\} \exp \{i \beta \sin (\phi / n) \sinh (a / n)\},
$$

see (7.9).

We now note that, with $\theta=\phi / n$,

$$
\begin{aligned}
d \mu_{\beta, \Omega}(\theta) & \equiv d \mu_{\tilde{I}_{\beta}, \Omega}(\phi) \\
& =\tilde{N}_{\Omega}^{-1} \prod_{\substack{|i-j|=1 \\
i \in \Omega}} \tilde{I}_{\beta}(\phi(i)-\phi(j)) \prod_{j \in \Omega} d \phi(j),
\end{aligned}
$$

$\phi(j) \in[-\pi n, \pi n]$, i.e. the properties of the measure $d \mu_{\tilde{I}_{\beta}, \Omega}(\phi)$ are very similar to the ones of $d \mu_{I_{\beta}, \Omega}(\phi)$ considered in Sect. 6. Let $d \mu_{\tilde{I}_{\beta}}(\phi)=d \mu_{\beta}(\theta)$ denote the limiting measure, as $\Omega \nearrow \mathbb{Z}^{2}$. We now prove

Theorem 7.2. Given $\beta<\infty$ and $q \in(0,1)$, there exists some finite $n_{0}(\beta, q)$ such that for all $n \geqq n_{0}(\beta, q)$ and all $\xi$, with $\min (\xi / n, 1-\xi / n) \geqq q$,

$$
\begin{aligned}
\left\langle e^{i \xi\left(\theta_{0}-\theta_{x}\right)}\right\rangle_{\Lambda}^{(n)}(\beta)= & \left(Z_{\Lambda}^{(n)}\right)^{-1} \sum_{\mathscr{N} \in \mathscr{F}} c_{\Lambda} \varrho_{\mathscr{N}} \int\left[z\left(\beta, \xi^{\prime} \varrho_{0}\right) F_{\beta}\left(\xi^{\prime} \varrho_{0} ; \nabla \phi\right)\right. \\
& \left.+z\left(\beta, \xi^{\prime} \varrho_{0}+\varrho^{\mathscr{N}}\right) F_{\beta}\left(\xi^{\prime} \varrho_{0}+\varrho^{\mathscr{N}} ; \nabla \phi\right)\right] \\
& \cdot \prod_{\varrho \in \mathscr{N}}\left[1+z(\beta, \varrho) F_{\beta}(\varrho ; \nabla \phi)\right] d \mu_{\tilde{I}_{\beta}}(\phi),
\end{aligned}
$$

where

$$
F_{\beta}(\varrho ; \nabla \phi)=F_{1, \beta}(\varrho ; \nabla \phi) \sin \phi(\varrho)+F_{2, \beta}(\varrho ; \nabla \phi) \cos \phi(\varrho),
$$

and the functions $F_{1, \beta}$ and $F_{2, \beta}$ have properties (1) through (3) listed in Theorem 6.1, in particular $\left\|F_{i, \beta}(\varrho ; \cdot)\right\|_{\infty} \leqq 1$.

Moreover,

$$
z(\beta, \varrho)=K(\varrho) \exp \left[-L(\beta, n) E_{1 \mathrm{lc}}(\varrho)\right]
$$


with

$$
E_{\mathrm{loc}}(\varrho) \geqq C\|\varrho\|_{1}+D \sum_{k=1}^{\infty}\left|\mathscr{S}_{k}^{\prime}(\varrho)\right|,
$$

for some positive constants $C$ and $D$ (with notations as in Theorem 6.1). The function $L(\beta, n)$ satisfies

$$
L(\beta, n) \geqq K n-L \beta
$$

for some positive constants $K$ and $L$.

The estimates on $z\left(\beta, \xi^{\prime} \varrho_{0}\right)$ and $z\left(\beta, \xi^{\prime} \varrho_{0}+\varrho^{\mathcal{N}}\right)$ are identical, but with $L(\beta, n)$ replaced by

$$
L_{q}(\beta, n) \geqq K_{q} n-L \beta \text {. }
$$

The Proof of Theorem 7.2 is very similar to the one of Theorem 4.1, see Sect. 4.4. It follows inductively from Lemmas 4.3 and 4.4 . By property ( $\tilde{\mathrm{c}})$ of $\tilde{I}_{\beta}$, the parameter $\varepsilon$ in Condition (4.10), Sect. 4.2, and Lemmas 4.3, 4.4 can be chosen to be e.g., $\varepsilon=n$, and the constant $c(\beta)$ in Condition (4.10) satisfies, in the present model,

$$
c(\beta) \leqq \beta / n^{2} \text {. }
$$

Lemma 4.4 can now be used to show that each square in $\mathscr{S}_{k}^{\prime}(\varrho), k=1,2,3, \ldots$, contributes an amount of at least

$$
\text { const } n-\operatorname{const}^{\prime} c(\beta) n^{2} \geqq c_{1} n-c_{2} \cdot \beta
$$

for some positive constants $c_{1}$ and $c_{2}$, to $L(\beta, n) E_{\text {loc }}(\varrho)$. (The inductive proof follows the one of Theorem 4.1.)

From Lemma 4.3 it follows that there is a contribution of at least

$$
\sum_{j \in \text { supp } \varrho}\left(c_{1} n-c_{2} \beta\right)|\varrho(j)|
$$

to $L(\beta, n) E_{\text {loc }}(\varrho)$ coming from the renormalization of $\cos \phi(\varrho)$ on scale $2^{0}$. From our estimates on $K(\varrho)$, see Theorems 2.1, 2.3, (7.6) and (7.10) follows, as usual, the upper bound

$$
z(\beta, \varrho) \leqq \exp \left[-\left(c^{\prime} L(\beta, n)-b^{\prime}\right)\|\varrho\|_{1}\right] \exp [-(c L(\beta, n)-b) A(\varrho)]
$$

provided $L(\beta, n) \geqq \max \left(b / c, b^{\prime} / c^{\prime}\right)$. See Theorem 5.3, Sect. 5.2, and Sect. 3. If $\varrho=\xi^{\prime} \varrho_{0}$, or $=\xi^{\prime} \varrho_{0}+\varrho^{\mathcal{N}}$ the function $L(\beta, n)$ must be replaced by $L_{q}(\beta, n)$ [since squares in $\mathscr{S}_{k}^{\prime}(\varrho)$ cover constituents of $\varrho$ whose total charge is only known to be $\geqq q$, rather than $\geqq 1$. See Theorem 2.3]. This completes the outline of the proof of Theorem 7.2.

We now turn to our proof of part (2) of Theorem 7.1. Since $\exp \beta \cos \theta$ is of positive type for all $\beta>0$, the measure $d \mu_{\beta}(\theta)=d \mu_{\tilde{I}_{\beta}}(\phi)$ is of positive type in $\phi=n \theta$. This property together with Theorem 7.2 permits us to complete the proof of Theorem 7.1, (2) by repeating the arguments described in Sect. 5.1, between (5.30) and (5.35) (upper bound on the fractional charge two-point correlation in the hard core Coulomb gas). It suffices to choose $n$ so large, depending on $\beta$ and $q$, that $c^{\prime} L_{q}(\beta, n)-b^{\prime}>0$ and $c L_{q}(\beta, n)-b>0$, and notice that $L(\beta, n) \geqq L_{q}(\beta, n)$. 
Remarks. 1. Because of properties $(\tilde{\mathrm{b}})$ through $(\tilde{\mathrm{d}})$ of $\tilde{I}_{\beta}$ and the behavior of the function $L(\beta, n)$, see (7.11), the proof of the lower bound (7.5) on the expectation of the disorder operator is almost identical to the proof of the analogous estimate (6.1) in the rotator model.

2. Our basic identity (7.9) stated in Theorem 7.2 exhibits a remarkable invariance property: It follows from the properties of the renormalization transformation outlined in the proof of Theorem 7.2 that the effective activity $z(\beta, \varrho)$ vanishes, unless the total charge, $Q(\varrho)$, of a multipole density $\varrho$ vanishes. Thus, from the explicit expression for the renormalized functions $F_{\beta}(\varrho ; \nabla \phi)$ it is easily seen that they are all invariant under the substitution

$$
\phi(j) \mapsto \phi(j)+n \alpha,
$$

i.e.,

$$
\theta_{j} \mapsto \theta_{j}+\alpha,
$$

where $\alpha$ is an arbitrary angle. This $U(1)$-invariance is obviously shared by $d \mu_{\tilde{I}_{\beta}}(\phi)$. The same $U(1)$-invariance appears in the renormalized expression for $\left\langle D_{0 x}^{\xi}\right\rangle^{(n)}(\beta)$ [see (6.15), (6.16) and Theorem 6.1, and replace $d \mu_{I_{\beta}}$ by $d \mu_{I_{\beta}}$. Thus, in the massless, intermediate phase of the $\mathbb{Z}_{n}$-models, an effective, continuous symmetry group, $U(1)$, appears, and suitably chosen correlations behave indeed like corresponding correlations in the low-temperature phase of the rotator model. This behavior contrasts the one for $\beta<\beta_{c}$ (unbroken $\mathbb{Z}_{n}$-symmetry) or $\beta>\bar{\beta}_{c}$ (broken $\mathbb{Z}_{n}$-symmetry), where connected correlations have exponential fall-off.

In renormalization group language, a fixed point of our renormalization transformations, for $\underline{\beta}_{c}<\beta<\bar{\beta}_{c}$, has a continuous symmetry group, $U(1)$, not found in the original model.

Similar phenomena are observed in the three- and four-dimensional $\mathbb{Z}_{n}$ lattice gauge theories, where one analyzes the expectations of the Wilson loop and the disorder operator. An analysis of these theories will appear in [17].

\subsection{The Solid-on-Solid Model}

This final section contains the proof that the order parameter, $\phi$, in the twodimensional solid-on-solid model has, at high temperatures, logarithmically divergent fluctuations. The main result is a variant of Theorem 5.1, and its proof consists of a combination of the arguments in the proof of Theorem 5.1 and in Sect. 6, involving just one additional, technical idea which we discuss below.

As in Sects. 5, 6 for the dual Villain and rotator models, we start by expressing the solid-on-solid model as a perturbation of a zero-mass measure by operators which, for small $\beta$, are "irrelevant". The construction of the zero-mass measure requires looking for a function $\mathbf{I}_{\beta}(\phi)$ which is analytic and nonzero in the strip $|\operatorname{Im} \phi|<\operatorname{const} \beta^{-1 / 3}$ and fulfills the following four conditions [see also Condition (4.10), Sect. 4.2, Sect. 6, conditions (a)-(d), Sect. 7.1 conditions $(\tilde{b})-(\tilde{d})]$ :

(a) $\mathbf{I}_{\beta}(\phi=n)=e^{-\beta|n|}$, for all $n \in \mathbb{Z}$.

(b) $\mathbf{I}_{\beta}$ is even and real on the real axis. 
(c) $\left|\mathbf{I}_{\beta}(\phi+i a) / \mathbf{I}_{\beta}(\phi)\right| \leqq e^{\beta^{1 / 3} g(a)}$, for all $a$ with $|a| \leqq \operatorname{const}^{\prime} \beta^{-1 / 3}$.

(d) $\left|\frac{d^{m}}{d \phi^{m}} \log \left(\mathbf{I}_{\beta}(\phi+i a) / \mathbf{I}_{\beta}(\phi)\right)\right| \leqq C \beta^{1 / 3} g(a)$ for some constant $C$ independent of $\phi$ and $\beta$, all $a$ with $|a| \leqq$ const $^{\prime} \beta^{-1 / 3}$, and $m=1,2$. Here $g(a)$ is as in Sect. 6 and Appendix B. The existence of a function, $\mathbf{I}_{\beta}$, with properties (a) through (d) is established in Appendix C.

We now construct an "unperturbed, zero-mass" measure, $d \mu_{\mathbf{I}_{\beta}}(\phi)$, as a vague limit of the measures

$$
d \mu_{\mathbf{I}_{\beta}, \Omega}(\phi)=\mathbf{N}_{\Omega}^{-1} \prod_{\substack{|i-j|=1 \\ i \in \Omega}} \mathbf{I}_{\beta}(\phi(i)-\phi(j)) \prod_{k \in \Omega} d \phi(k),
$$

when $\Omega \nearrow \mathbb{Z}^{2}[\phi(j)=0$, for $j \notin \Omega]$.

The measure $d \mu_{\mathbf{I}_{\beta}}$ can be shown to have properties (6.3) and (6.4) (with $\mathbf{I}_{\beta}$ replacing the function $I_{\beta}$ used in Sect. 6).

The equilibrium measure of the s-o-s model in a finite region $\Lambda \subset \mathbb{Z}^{2}$ with free boundary conditions at $\partial \Lambda$ is given by

$$
\mathbf{Z}_{\Lambda}^{-1} \prod_{j \in \Lambda}\left[1+2 \sum_{q=1}^{\infty} \cos (2 \pi q \phi(j))\right] d \mu_{\mathbf{I}_{\beta}}(\phi) .
$$

Let $\left\langle\cdot{ }_{\Lambda}^{0}(\beta)\right.$ denote the expectation in this measure. (The results and proofs which follow could be extended to Dirichlet and periodic b.c., as well.) Our main result for the s-o-s model is

Theorem 7.3. For $\beta$ sufficiently small,

$$
\left\langle e^{\varepsilon[\phi(0)-\phi(x)]}\right\rangle_{A}^{0}(\beta) \geqq e^{\left(\varepsilon^{2} / \beta^{\prime}\right) \ln (1+|x|)},
$$

where $\beta^{\prime}=\beta^{\prime}(\beta)$ tends to 0 , as $\beta$ tends to 0 .

Remarks. 1) By expanding to second order in $\varepsilon$ one deduces from Theorem 7.3

$$
\left\langle[\phi(0)-\phi(x)]^{2}\right\rangle_{A}^{0}(\beta) \geqq\left(1 / \beta^{\prime}\right) \ln (1+|x|),
$$

if $\beta$ is small enough.

2) It follows from a standard low-temperature (Peierls contour) expansion that $\left\langle[\phi(0)-\phi(x)]^{2}\right\rangle_{\Lambda}^{0}(\beta)$ is bounded uniformly in $\Lambda$ and in $x \in \Lambda$, provided $\beta$ is large. This and (7.18) establish the existence of a roughening transition, as $\beta$ is varied.

3) The interest in the s-o-s model is motivated by a heuristic argument suggesting that $\phi(\cdot)$ behaves like the graph of some type of interface in a threedimensional Ising model (with "plus-minus" b.c.) [10] or the random surface bounded by a planar Wilson loop in a lattice gauge theory [18].

Outline of Proof of Theorem 7.3. The first step in the proof consists again in applying Theorem 2.1 to $\prod_{j \in \Lambda}\left[1+2 \sum_{q=1}^{\infty} \cos (2 \pi q \phi(j))\right]$. As in Sects. 5 and 6 this yields

$$
\prod_{j \in \Lambda}\left[1+2 \sum_{q=1}^{\infty} \cos (2 \pi q \phi(j))\right]=\sum_{\mathscr{N} \in \mathscr{F}_{\Lambda}} c_{\mathscr{N}} \prod_{\varrho \in \mathscr{N}}(1+K(\varrho) \cos \phi(\varrho)),
$$


where each charge density $\varrho$ takes values in $2 \pi \mathbb{Z}$,

$$
K(\varrho) \leqq\left\{\prod_{j \in \text { suppe }} z(\varrho(j) \mid)\right\} e^{S(\varrho)},
$$

with $S$ as in Theorem 2.1 and

$$
z(2 \pi|q|) \leqq \text { const } e^{\varepsilon|q|},
$$

and $c_{\mathscr{N}}>0$, for all $\mathscr{N} \in \mathscr{F}_{\Lambda}$. Thus

$$
\left\langle e^{\varepsilon[\phi(0)-\phi(x)]}\right\rangle_{\Lambda}^{0}(\beta)=\mathbf{Z}_{\Lambda}^{-1}\left\{\sum_{\mathcal{N} \in \mathscr{F}_{A}} c_{\mathscr{N}} \mathbf{Z}_{\mathscr{N}}(\varepsilon ; 0, x)\right\},
$$

where

$$
\mathbf{Z}_{\mathcal{N}}(\varepsilon ; 0, x)=\int e^{\varepsilon[\phi(0)-\phi(x)]} \prod_{\varrho \in \mathcal{N}}(1+K(\varrho) \cos \phi(\varrho)) d \mu_{\mathbf{I}_{\beta}}(\phi) .
$$

Next, we make the change of variables

$$
\phi(j) \mapsto \phi(j)+\sigma_{\beta}(j),
$$

where

$$
\sigma_{\beta}(j)=\sigma(\beta) \varepsilon[C(j)-C(j-x)],
$$

$\sigma(\beta)$ is some function of $\beta$ to be chosen later, and $C$ is the Green's function of the finite difference Laplacian; see Sect. 5, (5.7), (5.8). We then find

$$
\mathbf{Z}_{\mathcal{N}}(\varepsilon ; 0, x)=\exp \left[2 \varepsilon^{2} \sigma(\beta)(C(0)-C(x))\right] \mathbf{Z}_{\mathcal{N}}^{\prime}\left(\sigma_{\beta}\right),
$$

where

$$
\begin{aligned}
\mathbf{Z}_{\mathcal{N}}^{\prime}\left(\sigma_{\beta}\right)= & \int e^{\varepsilon[\phi(0)-\phi(x)]} \prod_{\varrho \in \mathcal{N}}\left[1+K(\varrho) \cos \left(\phi(\varrho)+\sigma_{\beta}(\varrho)\right)\right] \\
& . \mathscr{I}_{\beta}\left(\nabla\left(\phi+\sigma_{\beta}\right)\right) d \mu_{\mathbf{I}_{\beta}}(\phi),
\end{aligned}
$$

and

$$
\mathscr{I}_{\beta}\left(\nabla\left(\phi+\sigma_{\beta}\right)\right)=d \mu_{\mathbf{I}_{\beta}}\left(\phi+\sigma_{\beta}\right) / d \mu_{\mathbf{I}_{\beta}}(\phi) .
$$

The derivation of (7.24) is as in Sect. 5, (5.12) and Sect. 6, (6.15) and (6.16).

Next, we carry out the renormalization of $\mathbf{Z}_{\mathcal{N}}^{\prime}\left(\sigma_{\beta}\right)$ by applying Lemmas 4.3 and 4.4 inductively, as in the proof of Theorem 4.1, Sect. 4.4. All renormalization transformations are done by means of complex translations,

$$
\phi(j) \mapsto \phi(j)+i a_{\varrho, \ell}(j),
$$

where $\ell$ labels charged constituents of a charge density $\varrho \in \mathcal{N}$ on all possible length scales. As remarked in Sect. 6, the supports of the functions $\nabla a_{Q, \ell}$ are pairwise disjoint, and $\left|a_{\varrho, \ell}(j)\right| \leqq$ const $^{\prime} \beta^{-1 / 3}$, for all $j, \varrho \in \mathcal{N}$ and $\ell$. Moreover they are chosen to be independent of the observable, $e^{\varepsilon[\phi(0)-\phi(x)]}$, i.e. of $\varepsilon$ and $\sigma_{\beta}$. We set

By Lemmas 4.3 and 4.4

$$
a_{\varrho}=\sum_{\ell} a_{\varrho, \ell} .
$$

$$
\operatorname{supp} a_{\varrho} \subseteq D(\varrho),
$$


where $D(\varrho)$ is the disk of radius at most $2 d(\varrho)+1$ containing supp $\varrho$ which was introduced in Sect. 4.

Let $\mathscr{N}_{0, x} \subseteq \mathscr{N}$ be the sub-ensemble of all those charge densities, $\varrho$, with the property that

$$
D(\varrho) \cap\{0, x\} \neq \emptyset .
$$

After renormalization, corresponding to the change of variables $(7.25), e^{\varepsilon[\phi(0)-\phi(x)]}$ is replaced by

$$
e^{\varepsilon[\phi(0)-\phi(x)]} \prod_{\varrho \in \mathscr{N}_{0, x}} e^{i \alpha_{\varrho}},
$$

where $\alpha_{\varrho}=\varepsilon\left(a_{\varrho}(0)-a_{\varrho}(x)\right)$. For, by (7.27), $a_{\varrho}(0)=a_{\varrho}(x)=0$, for all $\varrho \in \mathscr{N} \backslash \mathscr{N}_{0, x}$. After having carried out all the renormalization transformations we obtain

$$
\begin{aligned}
\mathbf{Z}_{\mathscr{N}}^{\prime}\left(\sigma_{\beta}\right)=\int e^{\varepsilon[\phi(0)-\phi(x)]} \prod_{\varrho \in \mathscr{N}_{0, x}}\left[1+z(\beta, \varrho) F_{\beta, \varepsilon}\left(\varrho ; \nabla\left(\phi+\sigma_{\beta}\right)\right)\right] \\
\prod_{\varrho \in \mathscr{N} \mathscr{N}_{0, x}}\left[1+z(\beta, \varrho) F_{\beta}\left(\varrho ; \nabla\left(\phi+\sigma_{\beta}\right)\right)\right] \mathscr{I}_{\beta}\left(\nabla\left(\phi+\sigma_{\beta}\right)\right) d \mu_{\mathbf{I}_{\beta}}(\phi),
\end{aligned}
$$

where

$$
\begin{aligned}
& F_{\beta, \varepsilon}(\varrho ; \nabla \phi)=i F_{1, \beta, \varepsilon}(\varrho ; \nabla \phi) \sin \phi(\varrho)+F_{2, \beta, \varepsilon}(\varrho ; \nabla \phi) \cos \phi(\varrho), \\
& F_{\beta} \equiv F_{\beta, 0}, F_{n, \beta} \equiv F_{n, \beta, 0},
\end{aligned}
$$

and

$$
\begin{aligned}
2 F_{n, \beta, \varepsilon}(\varrho, \nabla \phi) \equiv e^{i \alpha_{\varrho}} \prod_{\substack{j \in D(\varrho) \\
\kappa=1,2}} i_{\beta}\left(\partial_{\kappa} a_{\varrho}(j) ; \partial_{\kappa} \phi(j)\right) \\
+(-1)^{n} e^{-i \alpha_{\varrho}} \prod_{\substack{j \in D(\varrho) \\
\kappa=1,2}} i_{\beta}\left(-\partial_{\kappa} a_{\varrho}(j) ; \partial_{\kappa} \phi(j)\right)
\end{aligned}
$$

and the functions $F_{1, \beta, \varepsilon}$ and $F_{2, \beta, \varepsilon}$ have the same properties (1) through (4), Theorem 6.1, as the ones introduced for the rotator model in Sect. 6, in particular

$$
\left\|F_{\beta, \varepsilon}(\varrho ; \nabla \phi)\right\|_{\infty} \leqq 1 .
$$

Moreover, Theorem 6.1 holds for $\mathbf{Z}_{\mathcal{N}}^{\prime}\left(\sigma_{\beta}\right)$ if $\beta$ is replaced by $\beta^{-1}$. Since the functions $a_{\varrho}$ are independent of $\varepsilon$ and $\sigma_{\beta}, F_{\beta}(\varrho ; \cdot)$ and $z(\beta, \varrho)$ are independent of $\varepsilon$, as well. Finally, one may show that, for $\beta$ small enough,

$$
0<z(\beta, \varrho)<\exp \left[-\left(c^{\prime \prime} L_{0}(\beta)-b^{\prime \prime}\right)\|\varrho\|_{1}\right] \exp \left[-\left(c L_{0}(\beta)-b\right) A(\varrho)\right],
$$

where $L_{0}(\beta)$ is some function which tends to $\infty$, as $\beta$ tends to $0\left[L_{0}(\beta)\right.$ behaves like the function $L\left(\beta^{-1}\right)$, defined in Sect. 6].

Now notice that, by (7.31),

$$
e^{\varepsilon[\phi(0)-\phi(x)]} \prod_{\varrho \in \mathscr{N} \backslash \mathscr{N}_{0, x}}\left[1+z(\beta, \varrho) F_{\beta}\left(\varrho ; \nabla\left(\phi+\sigma_{\beta}\right)\right)\right] \mathscr{I}_{\beta}\left(\nabla\left(\phi+\sigma_{\beta}\right)\right) d \mu_{\mathbf{I}_{\beta}}(\phi)
$$

is positive, and by (7.30)

$$
\begin{aligned}
& \prod_{\varrho \in \mathscr{N}_{0}, x}\left[1+z(\beta, \varrho) F_{\beta, \varepsilon}\left(\varrho, \nabla\left(\phi+\sigma_{\beta}\right)\right)\right] \\
& \quad \geqq\left(\prod_{\varrho \in \mathscr{N}_{0}, x} \frac{1-z(\beta, \varrho)}{1+z(\beta, \varrho)}\right)\left(\prod_{\varrho \in \mathscr{N}_{0, x}}\left[1+z(\beta, \varrho) F_{\beta}\left(\varrho ; \nabla\left(\phi+\sigma_{\beta}\right)\right)\right]\right) .
\end{aligned}
$$


We define

$$
\begin{aligned}
\mathbf{Z}_{\mathcal{N}}\left(\sigma_{\beta}\right)= & \int e^{\varepsilon[\phi(0)-\phi(x)]} \prod_{\varrho \in \mathscr{N}}\left[1+z(\beta, \varrho) F_{\beta}\left(\varrho ; \nabla\left(\phi+\sigma_{\beta}\right)\right)\right] \\
& \cdot \mathscr{I}_{\beta}\left(\nabla\left(\phi+\sigma_{\beta}\right)\right) d \mu_{\mathbf{I}_{\beta}}(\phi) .
\end{aligned}
$$

From (7.20)-(7.24), (7.29), and (7.32), (7.33) we conclude that

$$
\begin{aligned}
\left\langle e^{\varepsilon[\phi(0)-\phi(x)]}\right\rangle_{\Lambda}^{0}(\beta) \geqq & \exp \left[2 \varepsilon^{2} \sigma(\beta)(C(0)-C(x))\right] \\
& \cdot \mathbf{Z}_{\Lambda}^{-1}\left\{\sum_{\mathscr{N} \in \mathscr{F}_{\Lambda}} c_{\mathscr{N}}\left(\prod_{\varrho \in \mathscr{N}_{0, x}} \frac{1-z(\beta, \varrho)}{1+z(\beta, \varrho)}\right) \mathbf{Z}_{\mathcal{N}}\left(\sigma_{\beta}\right)\right\} .
\end{aligned}
$$

Thus, it remains to exhibit uniform (in $\mathscr{N}, \Lambda$, and $x \in \Lambda$ ) lower bounds on the last two factors in $\{\cdot\}$ on the right hand side of (7.34).

Since $\varepsilon[\phi(0)-\phi(x)]$ is odd in $\phi$, while

$$
\prod_{\varrho \in \mathscr{N}}\left[1+z(\beta, \varrho) F_{\beta}(\varrho ; \nabla \phi)\right] d \mu_{\mathbf{I}_{\beta}}(\phi)
$$

is even [see property (b) of $\mathbf{I}_{\beta}$ ], the arguments used in the proof of the lower bound (6.27) on $Z_{\mathcal{N}}^{\prime}(\sigma)$ in the rotator model, see Sect. 6, can be repeated in the present situation and yield

$$
\mathbf{Z}_{\mathscr{N}}\left(\sigma_{\beta}\right) \geqq \text { const } \exp \left\{-(\varepsilon \sigma(\beta))^{2} c(\beta)[C(0)-C(x)]\right\} \mathbf{Z}_{\mathscr{N}},
$$

with $\mathbf{Z}_{\mathscr{N}}=\mathbf{Z}_{\mathscr{N}}\left(\sigma_{\beta}=0\right)$, for $\beta$ small enough [so that $c^{\prime \prime} L_{0}(\beta)-b^{\prime \prime}>0, c L_{0}(\beta)-b>0$, see $(7.31)$, and $z(\beta, \varrho)<1 / 4$, for all $\varrho]$. Here $c(\beta)$ is some function of $\beta$ which tends to 0 , as $\beta \rightarrow 0$. For details, see Sects. 6 and 5.1, (5.13) through (5.26).

We now choose

$$
\sigma(\beta)=c(\beta)^{-1} .
$$

In order to complete the proof we must finally prove a lower bound on

$$
K\left(\mathscr{N}_{0, x}\right) \equiv \prod_{\varrho \in \mathscr{N}_{0, x}} \frac{1-z(\beta, \varrho)}{1+z(\beta, \varrho)}
$$

Clearly,

$$
K\left(\mathscr{N}_{0, x}\right)=\prod_{n=1}^{\infty} \prod_{\left\{\varrho \in \mathscr{N}_{0, x}: 2^{n} \leqq d(\varrho)<2^{n+1}\right\}} \frac{1-z(\beta, \varrho)}{1+z(\beta, \varrho)} .
$$

By Theorem 2.1, (2.4)

$$
\operatorname{card}\left\{\varrho \in \mathcal{N}_{0, x}: 2^{n} \leqq d(\varrho)<2^{n+1}\right\} \leqq 2,
$$

and by (7.31)

$$
\frac{1-z(\beta, \varrho)}{1+z(\beta, \varrho)} \geqq 1-(n+1)^{-p(\beta)},
$$

if $2^{n} \leqq d(\varrho)<2^{n+1}$, for some number $p(\beta)$ which tends to $\infty$, as $\beta$ tends to 0 . This follows from the divergence of $L_{0}(\beta)$, as $\beta \rightarrow 0$, and the standard lower bound, $A(\varrho)$ $\geqq$ const $\ln d(\varrho)$. 
Thus, for $\beta$ small enough,

$$
K\left(\mathscr{N}_{0, x}\right) \geqq K>0,
$$

uniformly in $\mathscr{N} \in \mathscr{F}_{\Lambda}, \Lambda$, and $x \in \Lambda$. Combination of (7.34) through (7.39) yields

$$
\left\langle e^{\varepsilon[\phi(0)-\phi(x)]}\right\rangle_{\Lambda}^{0}(\beta) \geqq \mathrm{const} K \exp \left[\varepsilon^{2} c(\beta)^{-1}(C(0)-C(x))\right] \mathbf{Z}_{\Lambda}^{-1}\left\{\sum c_{\mathscr{N}} \mathbf{Z}_{\mathscr{N}}\right\} .
$$

Since the product of the last two factors on the right hand side of $(7.40)$ is $=1$, the proof of Theorem 7.3 is now complete, with $\beta^{\prime}=c(\beta) \rightarrow 0$, as $\beta \rightarrow 0$.

\section{Appendix A. The Duality Transformation}

In this appendix we verify relation (1.37) for a system in a finite region $A$ with boundary conditions at $\partial \Lambda$ which are invariant under the symmetry transformation $\theta_{j} \rightarrow \theta_{j}+\alpha, 0 \leqq \alpha \leqq 2 \pi$, of the Hamilton function. This is no loss of generality, since in two dimensions all infinite volume equilibrium states can be obtained from such b.c. Included in that class of boundary conditions are the ones corresponding to what we called "free b.c." in the dual models which were imposed in Sects. 5 and 6.

For simplicity, we only consider rotator models in a rectangular box $\Lambda$, with free ( $\equiv 0$ Dirichlet) b.c. imposed at $\partial \Lambda$, but as noted at the end, our arguments extend to a general class of b.c. in a straightforward manner. With each ordered nearest neighbor pair $\langle i, j\rangle, i$ and $j$ in $A, j=i+e_{1}$ or $j=i+e_{2}$, where $e_{1}$ and $e_{2}$ are the lattice basis vectors, we associate a function $f_{i j}(\theta)$ which is smooth and periodic in $\theta$, with period $2 \pi$. We then consider the generalized partition function

$$
Z_{\Lambda}=\int \prod_{\langle i, j\rangle \in \Lambda} f_{i j}\left(\theta_{i}-\theta_{j}\right) \prod_{k \in \Lambda} d \theta_{k}
$$

Next, we represent each $f_{i j}$ by its Fourier series

$$
f_{i j}\left(\theta_{i}-\theta_{j}\right)=\sum_{n_{i j} \in \mathbb{Z}} \hat{f}_{i j}\left(n_{i j}\right) \exp \left[i n_{i j}\left(\theta_{i}-\theta_{j}\right)\right] .
$$

Here the $n_{i j}$ 's are integer-valued bond variables, and we adopt the convention that

$$
n_{i j}=-n_{j i}
$$

We abbreviate $i j$ by $b, b$ denoting the oriented bond corresponding to $\langle i, j\rangle$ and substitute the Fourier series of $f_{i j}$ into (A.1). This yields

$$
\begin{aligned}
Z_{\Lambda} & =\sum_{\left\{n_{b}\right\}} \prod_{b \subset \Lambda} \hat{f}_{b}\left(n_{b}\right) \int \exp \left[i \sum_{b} n_{b}\left(\theta_{i}-\theta_{j}\right)\right] \prod_{k \in \Lambda} d \theta_{k} \\
& =\sum_{\left\{n_{b}\right\}} \prod_{b \subset \Lambda} \hat{f}_{b}\left(n_{b}\right) \int \prod_{k \in \Lambda} \exp \left[i(\delta n)_{k} \theta_{k}\right] d \theta_{k} \\
& =\sum_{\left\{n_{b}: \delta n=0\right\}} \prod_{b \subset \boldsymbol{A}} \hat{f}_{b}\left(n_{b}\right) .
\end{aligned}
$$

The symbol $\delta n$, the divergence of $n$, is defined by

with $i$ and $k$ in $\Lambda$.

$$
(\delta n)_{k}=\sum_{i:|i-k|=1} n_{k i}
$$


Now we claim that there is a $1-1$, onto correspondence between bond variables $\{n: \delta n=0\}$ and variables $\left\{m_{p}\right\}$, where $\left\{m_{p}\right\}$ is a family of integer-valued variables indexed by the plaquettes (oriented unit squares) $p \subset A$, oriented clockwise. This correspondence is given by

$$
n_{i j}=n_{b}=m_{p}-m_{p^{\prime}}
$$

where $p$ and $p^{\prime}$ are neighboring plaquettes which have $b=\langle i, j\rangle$ as a common bond. Moreover, $p$ is the plaquette whose orientation agrees with the one of $b$, whereas the orientation of $p^{\prime}$ agrees with the one of $-b$. If $p$ is not contained in $\Lambda$, we set $m_{p}=0$.

We recall that the plaquettes $p$ form the sites of the dual lattice $\Lambda^{*} \cong \Lambda$ $+(1 / 2,1 / 2)$. By (A.2) and (A.3) we have

$$
\begin{aligned}
Z_{\Lambda} & =\sum_{\left\{m_{p}\right\}} \prod_{b \subset \Lambda} \hat{f}_{b}\left(m_{p}-m_{p^{\prime}}\right), \quad p \cap p^{\prime}=b \\
& =\sum_{\left\{m_{p}\right\}} \prod_{i, j \in \Lambda^{*}} \hat{f}_{b}\left(m_{i}-m_{j}\right),
\end{aligned}
$$

where $\langle i, j\rangle$ is a pair of nearest neighbors in $\Lambda^{*}$ and $b$ the bond in $\Lambda$ dual to $\langle i, j\rangle$. The condition $m_{j}=0, j \notin \Lambda^{*}$ corresponds to Dirichlet boundary conditions at $\partial \Lambda^{*}$.

In order to obtain the partition function of the Villain or rotator model one sets

$$
f_{i j}(\theta)=f(\theta)
$$

where

$$
f(\theta)=v_{\beta}(\theta) \quad \text { or } \quad r_{\beta}(\theta)
$$

Next, we choose a path $\omega$ from 0 to $x$. The unnormalized correlation $Z_{\Lambda}\left\langle e^{i \xi\left(\theta_{0}-\theta_{x}\right)}\right\rangle_{\beta, \Lambda}$ is obtained by setting

$$
f_{i j}(\theta)=f(\theta) \exp \left[-i \chi_{i j} \xi \theta\right],
$$

with $\chi_{i j}=1$ if $(i, j)$ is a pair of nearest neighbors contained in $\omega$, oriented according to $\omega$, and $\chi_{i j}=0$, otherwise. Here we are using the simple fact that, with this definition of $\chi_{i j}$,

$$
-\sum_{i, j} \chi_{i j}\left(\theta_{i}-\theta_{j}\right)=\theta_{0}-\theta_{x}
$$

Let $\hat{\chi}_{i j}=1,(-1)$ if $\langle i, j\rangle,(\langle j, i\rangle)$ is an ordered nearest neighbor pair in $\Lambda^{*}$ dual to a bond in the path $\omega$, i.e. crossing $\omega$, and $\hat{\chi}_{i j}=0$, otherwise. Then

$$
\hat{f}_{b}\left(m_{i}-m_{j}\right)=\hat{f}\left(m_{i}-m_{j}+\xi \hat{\chi}_{i j}\right) \text {. }
$$

Thus (1.37) follows.

Finally we verify that the correspondence given by (A.3) is one to one and onto. Let $b$ be a bond belonging to $\partial \Lambda$, such that $b=p \cap p^{\prime}$ with $p \subset \Lambda$ and $p^{\prime} \not \Lambda$. Since $m_{p^{\prime}}=0$, (A.3) shows that $m_{p}= \pm n_{b}$. The plus (minus) sign is chosen if the orientation of $p$ agrees (disagrees) with that of $b$. Proceeding inductively (A.3) allows us to successively determine $m_{p}$, given $\left\{n_{b}\right\}$, provided that the equations are consistent. Consider a site $j \in \Lambda$, there are four bonds and four plaquettes containing $j$ as shown below. 
Fig. 2
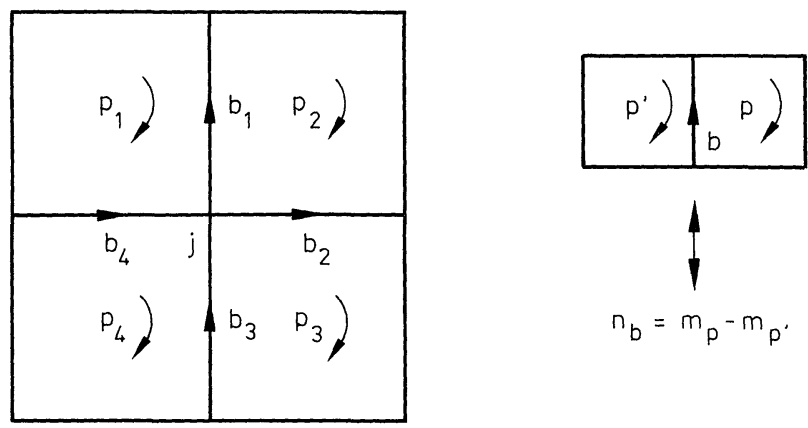

Assume e.g. that $m_{p_{1}}$ has already been determined, and $n_{b_{1}}, \ldots, n_{b_{4}}$ are given. Then by (A.3)

$$
\begin{aligned}
& m_{p_{2}}=n_{b_{1}}+m_{p_{1}}, \\
& m_{p_{4}}=n_{b_{4}}+m_{p_{1}} .
\end{aligned}
$$

We must check that there is a consistent choice for $m_{p_{3}}$, i.e. that

$$
m_{p_{3}} \equiv n_{b_{2}}+m_{p_{2}}=n_{b_{3}}+m_{p_{4}}
$$

or, using (A.5),

$$
n_{b_{2}}+n_{b_{1}}+m_{p_{1}}=n_{b_{3}}+n_{b_{4}}+m_{p_{1}}
$$

i.e.

$$
n_{b_{1}}+n_{b_{2}}-n_{b_{3}}-n_{b_{4}}=(\delta n)_{j}=0 .
$$

This completes the construction of $\left\{m_{p}\right\}$. Clearly, $\left\{m_{p}\right\}$ is unique, given $\left\{n_{b}\right\}$ and the b.c. $m_{p}=0$, for all $p \not \subset$. Conversely, given any such $\left\{m_{p}\right\}$, it determines a unique $\left\{n_{b}\right\}$, by setting

$$
n_{b}=m_{p}-m_{p^{\prime}}
$$

with $p, p^{\prime}$, and $b$ as shown above. It is obvious that $\delta n=0$.

We conclude this section with a comment on general boundary conditions invariant under the symmetry transformation $\theta_{j} \rightarrow \theta_{j}+\alpha$. Let

$$
\begin{gathered}
\bar{\Lambda}=\{j: \operatorname{dist}(j, \Lambda) \leqq 1\}, \\
\partial \bar{\Lambda}=\{j: \operatorname{dist}(j, \Lambda)=1\} .
\end{gathered}
$$

Let $\varrho\left(\theta_{\partial \bar{\Lambda}}\right)$ be a function of $\left\{\theta_{j}: j \in \partial \bar{\Lambda}\right\}$ with the property that

$$
\varrho\left(\theta_{\partial \bar{A}}\right)=\varrho\left((\theta+\alpha)_{\hat{o} \bar{\Lambda}}\right),
$$

where $\alpha_{j}=\alpha=$ const, for all $j \in \partial \bar{\Lambda}$. Let $\varrho$ denote the Fourier transform of $\varrho$. By (A.6),

$$
\hat{\varrho}\left(\left\{n_{j}\right\}_{j \in \partial \bar{\Lambda}}\right)=0 \text {, }
$$

unless

$$
\sum_{j \in \partial \bar{A}} n_{j}=0
$$


We now consider the generalized partition function

$$
Z_{\Lambda, e}=\int \prod_{\substack{\langle i, j\rangle \subset \bar{\lambda} \\\langle i, j\rangle\langle\bar{\Lambda}}} f_{i j}\left(\theta_{i}-\theta_{j}\right) \varrho\left(\theta_{\partial \bar{\Lambda}}\right) \prod_{k \in \bar{A}} d \theta_{k} .
$$

Given $j \in \partial \bar{\Lambda}$, let $b(j)$ be the bond containing $j$ and a site $i \in \Lambda$. Fourier transformation yields

$$
Z_{\Lambda, \varrho}=\sum_{\left\{n_{b}: \delta n=0\right\}} \prod_{b \subset \bar{A}} \hat{f}_{b}\left(n_{b}\right) \hat{\varrho}\left(\left\{n_{b(j)}\right\}_{j \in \partial \bar{A}}\right) .
$$

We leave it to the reader to check that, thanks to (A.7), there exists, given $\left\{n_{b}\right\}$, a unique integer-valued function $\left\{m_{p}\right\}$ such that

$$
n_{b}=m_{p}-m_{p^{\prime}}
$$

with $b$ compatible with the orientation of $p$, and

$$
m_{p_{0}}=0
$$

for some arbitrary, but fixed $p_{0} \subset \bar{\Lambda} \backslash \Lambda$.

\section{Appendix B. An Analytic Interpolation of $I_{\beta}(n)$, and Estimates on Bessel Functions}

In this appendix we establish the existence of a function, $I_{\beta}(\phi)$, with properties (a) through (d) required in Sect. 6 [between (6.2) and (6.3)]. For $\phi=n$, an integer,

$$
\begin{aligned}
I_{\beta}(n) & =\frac{1}{2 \pi} \int_{0}^{2 \pi} e^{\beta \cos \theta} e^{\mathrm{in} \theta} d \theta \\
& =\frac{1}{2 \pi} \int_{-\pi}^{\pi} e^{\beta \cos \theta} e^{\operatorname{in} \theta} d \theta
\end{aligned}
$$

is the $n^{\text {th }}$ modified Bessel function evaluated at the point $\beta \in \mathbb{R}_{+}$.

We propose to construct a suitable analytic interpolation, $I_{\beta}(\phi)$, defined for all $\phi$ in the strip $|\operatorname{Im} \phi| \leqq \beta / 2$, which is given by (B.1) when $\phi$ is an integer. Our first task is to evaluate $I_{\beta}(n)$ asymptotically for large $\beta$. We do this by using the method of steepest descent. Let $\theta_{c}$ be the critical point of $\beta \cos \theta+\operatorname{in} \theta$, i.e. $\theta_{c}$ is the solution of the equation

i.e.

$$
\beta \sin \theta_{c}=\text { in, }
$$

We change variables,

$$
\theta_{c}=i \sinh ^{-1}\left(\frac{n}{\beta}\right) \equiv i r
$$

$$
\theta \rightarrow \theta+i r
$$

in Eq. (B.1). This change of variables corresponds to a deformation of the integration contour. The contributions of the vertical sides of the contour cancel by periodicity. Thus we obtain

$$
I_{\beta}(n)=\frac{e^{-n r}}{2 \pi} \int_{-\pi}^{\pi} e^{\beta \cos \theta \cdot \cosh r} e^{-i \beta \sinh r \cdot \theta^{3} h(\theta)} d \theta,
$$


where $h(\theta)=\theta^{-3}(\sin \theta-\theta)$. The range of integration is now broken up into two pieces,

$$
\left[-\frac{\pi}{2}, \frac{\pi}{2}\right] \text { and }\left[-\pi,-\frac{\pi}{2}\right] \cup\left[\frac{\pi}{2}, \pi\right] \text {. }
$$

This yields

$$
I_{\beta}(n)=\frac{e^{-n r}}{2 \pi} \int_{-\pi / 2}^{\pi / 2} e^{\beta \cos \theta \cosh r} e^{-i \beta \sinh r \theta^{3} h(\theta)} d \theta+\varepsilon .
$$

Since $\cos \theta \leqq 0$, for $\theta \in\left[-\pi,-\frac{\pi}{2}\right] \cup\left[\frac{\pi}{2}, \pi\right]$,

We set

$$
\varepsilon<\frac{e^{-n r}}{2}
$$

$$
\left.\begin{array}{c}
\sigma=(\beta \cosh r)^{1 / 2}=\left(\beta \sqrt{1+\frac{n^{2}}{\beta^{2}}}\right)^{1 / 2} \\
g(\theta)=\theta^{-4}\left(\cos \theta-1+\frac{\theta^{2}}{2}\right) \\
X(\theta)=\sigma^{-2} \theta^{4} g\left(\sigma^{-1} \theta\right)+i \beta \sinh r \sigma^{-3} \theta^{3} h\left(\sigma^{-1} \theta\right)
\end{array}\right\}
$$

If we change variables, $\theta \rightarrow \sigma^{-1} \theta$, we get

$$
I_{\beta}(n)=\frac{e^{-n r}}{2 \pi \sigma} e^{\sigma^{2}} \int_{-\pi \sigma / 2}^{\pi \sigma / 2} e^{-\theta^{2} / 2} e^{X(\theta)} d \theta+\varepsilon .
$$

Next, we extract the leading contribution to the first term on the right side of (B.7) by using Taylor's theorem with remainder,

$$
e^{X(\theta)}=1+X(\theta)+\int_{0}^{1} e^{s X(\theta)}(1-s) X(\theta)^{2} d s .
$$

It is straightforward to show that

$$
\int_{-\pi \sigma / 2}^{\pi \sigma / 2} e^{-\theta^{2} / 2}(1+X(\theta)) d \theta=\sqrt{2 \pi}\left(1+O\left(\sigma^{-2}\right)\right),
$$

because $g$ and $h$ are bounded. Moreover, since

for

$$
-s|X(\theta)|+\frac{\theta^{2}}{2}>\frac{\theta^{2}}{4}
$$

$$
\begin{gathered}
\theta \in[-\sigma \pi / 2, \sigma \pi / 2], \text { for all } 0 \leqq s \leqq 1, \\
\int_{-\pi \sigma / 2}^{\pi \sigma / 2} e^{-\theta^{2} / 2+s X(\theta)} X(\theta)^{2} d \theta=O\left(\sigma^{-2}\right)
\end{gathered}
$$

Thus

$$
\begin{aligned}
I_{\beta}(n) & =\frac{e^{-n r}}{\sqrt{2 \pi} \sigma} e^{\sigma^{2}}\left(1+O\left(\sigma^{-2}\right)\right)+\varepsilon \\
& =\frac{e^{-n r}}{\sqrt{2 \pi} \sigma} e^{\sigma^{2}}\left(1+O\left(\sigma^{-2}\right)\right),
\end{aligned}
$$


by (B.5). We define

$$
L_{\beta}(n)=\frac{1}{\sqrt{2 \pi}}\left[\beta\left(1+\frac{n^{2}}{\beta^{2}}\right)^{1 / 2}\right]^{-1 / 2} \exp \left[-n \sinh ^{-1}\left(\frac{n}{\beta}\right)+\beta\left(1+\frac{n^{2}}{\beta^{2}}\right)^{1 / 2}\right] .
$$

By (B.2), (B.6), and (B.11)

$$
I_{\beta}(n)=L_{\beta}(n)\left(1+O\left(\sigma^{-2}\right)\right),
$$

for all $n \in \mathbb{Z}$. We note that $L_{\beta}(n)$ is the restriction of the function

$$
L_{\beta}(\phi)=\frac{1}{\sqrt{2 \pi}}\left[\beta\left(1+\frac{\phi^{2}}{\beta^{2}}\right)^{1 / 2}\right]^{-1 / 2} \exp \left[-\phi \sinh ^{-1}\left(\frac{\phi}{\beta}\right)+\beta\left(1+\frac{\phi^{2}}{\beta^{2}}\right)^{1 / 2}\right]
$$

which is analytic in the strip $|\operatorname{Im} \phi| \leqq \beta / 2$, to the integers. Using Taylor's theorem one verifies that

$$
\left|L_{\beta}(\phi+i a)\right| \leqq e^{\text {const } a^{2} / \beta} L_{\beta}(\phi)
$$

for real $\phi$ and $|a| \leqq \beta / 2$.

We now define

$$
I_{\beta}(\phi)=L_{\beta}(\phi) E_{\beta}(\phi)
$$

where

$$
E_{\beta}(\phi)=\exp \left[\sum_{n}\left(\ln I_{\beta}(n)-\ln L_{\beta}(n)\right) \frac{\sin ^{2}[(\phi-n) \pi]}{(\phi-n)^{2} \pi^{2}}\right] .
$$

The proof of properties (a)-(d) follows easily from (B.12)-(B.14).

\section{Appendix C. An Analytic Interpolation of $e^{-\beta|n|}$}

Here we construct an analytic interpolation of the function $e^{-\beta|n|}, n \in \mathbb{Z}$. The basic idea is as in Appendix B, but the present construction is considerably simpler than the one in Appendix B, so that we only sketch it. We define

$$
\begin{aligned}
& \mathbf{L}_{\beta}(\phi)=\exp \left[-\beta^{2 / 3}\left(1+\beta^{2 / 3} \phi^{2}\right)^{1 / 2}\right], \\
& \mathbf{E}_{\beta}(\phi)=\exp \left[-\sum_{n}\left(\beta|n|-\beta^{2 / 3}\left(1+\beta^{2 / 3} n^{2}\right)^{1 / 2}\right) \frac{\sin ^{2}[(\phi-n) \pi]}{(\phi-n)^{2} \pi^{2}}\right], \\
& \mathbf{I}_{\beta}(\phi)=\mathbf{L}_{\beta}(\phi) \mathbf{E}_{\beta}(\phi) .
\end{aligned}
$$

Note that $\mathbf{I}_{\beta}(\phi)$ is real and even, for $\phi \in \mathbb{R}$, and analytic in the strip $|\operatorname{Im} \phi|$ $\leqq 1 / 2 \beta^{-1 / 3}$. Moreover,

$$
|\beta| n\left|-\beta^{2 / 3}\left(1+\beta^{2 / 3} n^{2}\right)^{1 / 2}\right| \leqq \frac{\beta^{1 / 3}}{2|n|} .
$$

Properties (c) and (d) of $\mathbf{I}_{\beta}$ required in Sect. 7.2 then follow directly from (C.1), (C.2) and Taylor's theorem with remainder. 


\section{Appendix D. Boundary Conditions}

For simplicity we have proved our main theorems for the case of free boundary conditions in models dual to the Villain or classical rotator. In this appendix we briefly indicate how to deal with Dirichlet conditions on the boundary of a large square $\Lambda$. First we must modify our construction of the ensembles $\mathscr{N}$ appearing in Sect. 2. This is because a long dipole of length $\ell$ both of whose charges are near the boundary of $A$ has small energy $\approx O(1)$ compared to its entropy $\approx \ln \ell$. In order to avoid forming such a dipole we shall imagine that a charged $\varrho$ is "neutral" by associating opposite image charges via reflection through one of the boundaries of $\Lambda$. Our ensemble $\mathscr{N}$ may now have many @'s localized near the boundary of $\Lambda$ for which $Q(\varrho) \neq 0$.

We make the above idea more precise as follows. Let us define

$$
d_{\Lambda}(\varrho) \equiv\left[\begin{array}{lll}
\max \left[\operatorname{dist}\left(\varrho, \Lambda^{c}\right), d(\varrho)\right], & \text { if } \quad Q(\varrho) \neq 0 \\
d(\varrho), & \text { if } \quad Q(\varrho)=0 .
\end{array}\right.
$$

Note that the (Dirichlet) electrostatic energy of $\varrho$ is at least as large as $\ln d_{\Lambda}(\varrho)$. The revised version of Theorem 2.1 reads as before except that $\mathcal{N}$ now satisfies:

a) If $\varrho, \varrho^{\prime} \in \mathscr{N}, \varrho \neq \varrho^{\prime}$ then $\operatorname{dist}\left(\varrho, \varrho^{\prime}\right) \geqq M\left[\min \left(d_{\Lambda}(\varrho), d_{\Lambda}\left(\varrho^{\prime}\right)\right)\right]^{\alpha}$.

b) If $\varrho_{1} \subset \varrho \in \mathscr{N}$ satisfies $\operatorname{dist}\left(\varrho_{1}, \varrho-\varrho_{1}\right) \geqq 2 M d\left(\varrho_{1}\right)^{\alpha}$,

then $Q\left(\varrho_{1}\right) \neq 0$ and

$$
2 M \operatorname{dist}\left(\varrho_{1}, \Lambda^{c}\right)^{\alpha} \geqq \operatorname{dist}\left(\varrho_{1}, \varrho-\varrho_{1}\right) .
$$

The bound on $K$ given by (2.6) and (2.7) holds with

$$
n(\varrho)=\text { smallest integer } \geqq \ln _{2}\left(M d_{\Lambda}(\varrho)^{\alpha}\right) \text {. }
$$

The proof of Theorem 2.1 proceeds as before. We iterate our application of Lemma 2.2 to subensembles $\mathscr{E}_{n}$ until conditions a) and b) are met. The ensembles $\mathscr{E}_{n}$ are $n$-ensembles with $\varrho \in \mathscr{E}_{n}$ if and only if for some $\varrho^{\prime} \in Q_{n}, \varrho \neq \varrho^{\prime}$

$$
\operatorname{dist}\left(\varrho, \varrho^{\prime}\right) \leqq M\left[\min \left(d_{\Lambda}(\varrho), d_{\Lambda}\left(\varrho^{\prime}\right)\right)\right]^{\alpha} \text {. }
$$

Conditions a) and b) above follow in a straightforward manner from this condition. The proof of our entropy estimate requires only small alterations.

In order to estimate the electrostatic energy (Sects. 3 and 4 ), for charged configurations we define $\mathscr{S}_{k}^{\prime}(\varrho, \Lambda) \subset \mathscr{S}_{k}(\varrho)$ to be the subcollection of $2^{k} \times 2^{k}$ squares which satisfy (3.3) and $\operatorname{dist}\left(s, \Lambda^{c}\right) \geqq 2^{k+2}$; when $\mathscr{S}_{k}(\varrho)$ consists of a single square we set $\mathscr{S}_{k}^{\prime}(\varrho, \Lambda)=\emptyset$ unless $Q(\varrho) \neq 0$ and $2^{k+3} \leqq \operatorname{dist}\left(\varrho, \Lambda^{c}\right)$ in which case $\mathscr{S}_{k}^{\prime}(\varrho) \equiv \mathscr{S}_{k}(\varrho)$. The statement of Theorem 4.1 reads as before except condition b) becomes

$$
d(\bar{\varrho}) \leqq d_{\Lambda}(\varrho)+d(\varrho) .
$$

Our proofs of the theorems in Sects. 5 and 6 are nearly the same except we must deal with a possible $\varrho$, with $Q(\varrho) \neq 0$ and $\varrho$ localized in some neighborhood of our observable. If we take $\Lambda$ very large in comparison to the support of our observable then it is easy to see that the effective activity of such a $\varrho$ is $\exp \left[-\beta^{\prime} \ln (\operatorname{dist}(\varrho, \partial \Lambda))\right]$ which is negligible. In general for $\beta$ sufficiently large it can be shown as in Sect. 3 that

$$
|z(\beta, \varrho)| \leqq \exp \left\{-\beta^{\prime}(\beta) \ln \left[d_{\Lambda}(\varrho)\right]\right\},
$$

where $\beta^{\prime}(\beta) \rightarrow \infty$ as $\beta \rightarrow \infty$. 
We conclude with a comment concerning the proof of Theorem 4.1. If $s \in \mathscr{S}_{k}^{\prime}\left(\varrho^{*}, \Lambda\right)$ and $\varrho \cap s \neq \emptyset$ then by our definition of $\mathscr{S}^{\prime}$ and conditions a) and b) above, $Q(\varrho)=0$. Thus the renormalization of charges explained in Sect. 4 proceeds with only minor changes since the $\varrho$ 's which interfere with the renormalization of $\varrho^{*}$ are still sparse and neutral.

\section{Appendix E. Properties of Components $C_{i}$}

We shall establish properties a) and b) of the components $C_{i}$ [following (4.37)] by induction on $d\left(\varrho_{i}\right)$. Clearly both a) and b) are valid if $d\left(\varrho_{i}\right) \leqq 2$. We first establish a). Let

$$
\mathscr{M}=\left\{\varrho \in S_{i} \mid d(\varrho) \geqq \frac{1}{2} d\left(\varrho_{i}\right)\right\}
$$

and define $C_{i j}$ to be the components of

$$
\bigcup_{\varrho \in S_{1}-\mathscr{M}} D(\varrho)
$$

By induction, diam $C_{i j} \leqq \frac{5}{2} d\left(\varrho_{i, j}\right)$. Since $C_{i j}$ intersects $D(\varrho)$ for some $\varrho \in \mathscr{M}$ we conclude by (2.4) that

$$
2 d\left(\varrho_{i}\right) \geqq \operatorname{dist}\left(\varrho, \varrho_{i, j}\right) \geqq M d\left(\varrho_{i, j}\right)^{\alpha} .
$$

Thus, for $M$ large, $d\left(\varrho_{i}\right) \gg d\left(\varrho_{i j}\right)$, for all $j$. If $\mathscr{M}$ has more than one element then, since $C_{i}$ is connected, there are two elements, $\varrho_{1}$ and $\varrho_{2}$, of $\mathscr{M}$ such that $D\left(\varrho_{1}\right)$ and $D\left(\varrho_{2}\right)$ are connected by some $C_{i j}$. Hence, by (2.4),

$$
\frac{5}{2} d\left(\varrho_{i j}\right) \geqq \operatorname{diam} C_{i j} \geqq \frac{M}{2}\left(\frac{d\left(\varrho_{i}\right)}{2}\right)^{\alpha}
$$

which contradicts the above inequality, for $M \gg 1$ and $\alpha>1$. Thus $\mathscr{M}$ has exactly one element, and

In order to establish b) assume

$$
\operatorname{diam} C_{i} \leqq 2 d\left(\varrho_{i}\right)+2 \max _{j} d\left(\varrho_{i, j}\right) \leqq \frac{5}{2} d\left(\varrho_{i}\right) .
$$

$$
\left|\partial C_{i j}\right| \leqq 10 d\left(\varrho_{i, j}\right)
$$

Hence

$$
\begin{aligned}
\left|\partial C_{i}\right| & \leqq\left|\partial D\left(\varrho_{i}\right)\right|+\sum_{j}\left|\partial C_{i, j}\right| \\
& \leqq 8 d\left(\varrho_{i}\right)+10 \sum_{j} d\left(\varrho_{i, j}\right) .
\end{aligned}
$$

By property a) and (2.4) we know that there are at most

$$
8 d\left(\varrho_{i}\right)\left[M 2^{\alpha k}\right]^{-1}
$$

components $C_{i j}$ of diameter $2^{k-1}$ [which intersect $\left.\partial D(\varrho)\right]$. Therefore the last term in the sum over $j$ is bounded above by

$$
80 \sum_{k}^{n_{l}} d\left(\varrho_{i}\right)\left[M 2^{\alpha k}\right]^{-1} \cdot 2^{k-1} \leqq 2 d\left(\varrho_{i}\right), \quad\left[n_{i} \leqq \alpha^{-1} \log _{2} d\left(\varrho_{i}\right)\right]
$$

for $M$ sufficiently large. 
Acknowledgements. We wish to thank J. Lebowitz and E. H. Lieb for some useful discussions and M. Wertheim for very valuable help in the construction of the analytic interpolations in Appendices B and C. T.S. thanks J. Glimm and A. Jaffe for helpful conversations concerning infinite number of distance scales and O. McBryan for help in understanding some estimates in the dual variables. We also wish to thank S. Breen, J. Imbrie, L. Rosen, B. Sharpe, and P. Sharpe for pointing out inaccuracies in a previous version of this paper. Finally, T.S. thanks N. Kuiper for invitations to I.H.E.S. and J.F. thanks A. Jaffe for an invitation to Harvard University. These visits facilitated our collaboration.

\section{References}

1. Berezinskii, V.L. : Sov. Phys. JETP 32, 493 (1971)

Kosterlitz, J.M., Thouless, D.J. : J. Phys. C 6, 1181 (1973)

Kosterlitz, J.M.: J. Phys. C 7, 1046 (1974)

Villain, J. : J. Phys. (Paris) 32, 581 (1975)

José, J.V., Kadanoff, L.P., Kirkpatrick, S., Nelson, D.R. : Phys. Rev. B 16, 1217 (1977)

2. Fröhlich, J., Spencer, T.: Phys. Rev. Lett. 46, 1006 (1981)

3. Brydges, D.C.: Commun. Math. Phys. 58, 313 (1978)

Brydges, D., Federbush, P.: Commun. Math. Phys. 73, 197 (1980)

4. Stillinger, F.H., Lovett, R.: J. Chem. Phys. 49, 1991 (1968)

5. Fröhlich, J., Spencer, T.: J. Stat. Phys. 24, 617 (1981)

6. Fröhlich, J., Park, Y.M.: Commun. Math. Phys. 59, 235 (1978)

7. Fröhlich, J., Isreal, R., Lieb, E.H., Simon, B. : J. Stat. Phys. 22, 297 (1980)

8. Siegert, A.J.F.: Physica 26, 30 (1960)

9. Fröhlich, J.: Commun. Math. Phys. 47, 233 (1976)

10. See e.g., Leamy, H.J., Gilmer, G.H., Jackson, K.A.: In: Surface physics of crystalline materials. Blakely, J.M. (ed.). New York: Academic Press 1976

Müller-Krumbhaar, H.: In : Crystal growth and materials. Kaldis, E., Scheel, H. (eds.). Dordrecht: North Holland 1977

van Beijeren, H.: Phys. Rev. Lett. 38, 993 (1977); and references given there

11. Ginibre, J.: Commun. Math. Phys. 16, 310 (1970)

12. Bricmont, J., Fontaine, J.-R., Landau, L.J.: Commun. Math. Phys. 56, 281 (1977)

13. Aizenman, M., Simon, B.: Phys. Lett. 76 A, 281 (1980)

14. McBryan, O., Spencer, T.: Commun. Math. Phys. 53, 299 (1977)

15. Elitzur, S., Pearson, R., Shigemitsu, J.: Phys. Rev. D 19, 3698 (1979)

16. Lanford, O.E., Ruelle, D.: Commun. Math. Phys. 13, 194 (1969)

17. Fröhlich, J., Spencer, T.: Submitted to Commun. Math. Phys.

18. Lüscher, M.: Symmetry breaking aspects of the roughening transition in gauge theories. DESY Preprint 80/81, Sept. 1980

Communicated by A. Jaffe

Received April 23, 1981 